\title{
A Many-Analysts Approach to the Relation Between Religiosity and Well-being
}

\author{
The MARP Team*广
}

\begin{abstract}
The relation between religiosity and well-being is one of the most researched topics in the psychology of religion, yet the directionality and robustness of the effect remains debated. Here, we adopted a many-analysts approach to assess the robustness of this relation based on a new cross-cultural dataset ( $N=10,535$ participants from 24 countries). We recruited 120 analysis teams to investigate (1) whether religious people self-report higher wellbeing, and (2) whether the relation between religiosity and self-reported well-being depends on perceived cultural norms of religion (i.e., whether it is considered normal and desirable to be religious in a given country). In a two-stage procedure, the teams first created an analysis plan and then executed their planned analysis on the data. For the first research question, all but 3 teams reported positive effect sizes with credible/confidence intervals excluding zero (median reported $\beta=0.120$ ). For the second research question, this was the case for $65 \%$ of the teams (median reported $\beta=0.039$ ). While most teams applied (multilevel) linear regression models, there was considerable variability in the choice of items used to construct the independent variables, the dependent variable, and the included covariates.
\end{abstract}

Keywords: Many Analysts, Religion, Health, Open Science

The relation between religion and well-being has been a topic of debate for centuries. While Freud considered religion a "universal obsessional neurosis" and Nietzsche 
called Christianity "the greatest misfortune of humanity", the recent scientific literature has painted a more positive picture of religion's effect on (mental) health (e.g., Gebauer et al., 2017; George et al., 2002; Koenig \& Larson, 2001; Plante \& Sherman, 2001; Seybold \& Hill, 2001; Thoresen, 1999; Zimmer et al., 2016). Individual religiosity has, for instance, been related to less depression (Smith et al., 2003), more happiness (Abdel-Khalek, 2006; Lewis \& Cruise, 2006), higher life satisfaction (Lim \& Putnam, 2010), and even lower mortality (Ebert et al., 2020; Stavrova, 2015). At the same time, the robustness, universality, and methodological specificity of the religion-well-being relation remains an outstanding question. In this project, we adopted a many-analysts approach to investigate two research questions using a new large cross-cultural dataset featuring $N=10,535$ participants from 24 countries. Specifically, we recruited 120 teams to conduct analyses in order to answer the following two research questions: (1) "Do religious people self-report greater well-being?", and (2) "Does the relation between religiosity and self-reported well-being depend on perceived cultural norms regarding religion?". In the subsequent sections, we will first introduce our theoretical framework, dataset, and the many-analysts approach, before describing the key results with respect to the stated research questions and the varying approaches taken by the many-analysts teams. A general discussion of the project and the results is included in the closing article (Hoogeveen et al., in preparation).

\section{Theoretical Background}

The literature on the psychology of religion is replete with positive correlations between (self-rated) religiosity and mental health (Abdel-Khalek, 2006; George et al., 2002; Koenig and Larson, 2001; Plante and Sherman, 2001; Seybold and Hill, 2001; Smith et al., 2003; Thoresen, 1999; Zimmer et al., 2016; see Koenig, 2009 for a review). At the same time, meta-analyses indicate that the relation between religion and well-being is often small (around $r=.1$; Bergin, 1983; Hackney and Sanders, 2003; Koenig and Larson, 2001).

\footnotetext{
*All authors with their affiliations appear at the end of this paper.

† Corresponding author: Suzanne Hoogeveen. E-mail: suzanne.j.hoogeveen@gmail.com.
} 
In addition, it has been argued that positive associations are found only for particular measures and operationalizations of these constructs (Hackney \& Sanders, 2003; Poloma \& Pendleton, 1989). A recent meta-analysis of longitudinal studies reported that, out of eight religiosity/spirituality measures, only participation in public religious activities and the importance of religion were statistically significantly related to self-rated mental health, which was operationalized as distress, life satisfaction, well-being, and quality of life (Garssen et al., 2020).

Furthermore, the type of religiosity (i.e., intrinsic vs extrinsic; positive vs. negative religious coping) and religious status (religious vs. uncertain) appear to moderate the relationship between religion and mental well-being (Smith et al., 2003; Villani et al., 2019). For instance, extrinsic religious orientation (i.e., when people primarily use their religious community as a social network, whereas personal religious beliefs are secondary) and negative religious coping (i.e., when people have internal religious guilt or doubts) have been shown to be negatively related to well-being (Abu-Raiya, 2013; Weber \& Pargament, 2014). Yet other research suggests that it is precisely the social aspect of religious service attendance and congregational friendships that explains how religiosity is positively associated with life satisfaction (Lim \& Putnam, 2010). Moreover, the direction of the religiosity-mental health relation remains unclear; while engaging in religious activities might make people happier, people with better mental health might also be more likely to engage in public, social events.

Additionally, there is large variability in the extent to which religion is ingrained in culture and social identity across the globe (Kelley \& de Graaf, 1997; Ruiter \& van Tubergen, 2009). Accordingly, when investigating the association between religiosity and well-being, it may be necessary to take into account the cultural norms related to religiosity within a society. Being religious may contribute to self-rated health and happiness when being religious is perceived to be a socially expected and desirable option (Diener et al., 2011; Ebert et al., 2020; Gebauer et al., 2017; Stavrova, 2015; Stavrova et al., 2013). This makes sense from the literature on person-culture fit (Dressler et al., 2007): a high 
person-culture fit indicates good agreement between one's personal values and beliefs and the beliefs that are shared by one's surrounding culture. A fruitful way to measure cultural norms is through the shared, intersubjective perception of the beliefs and attitudes that are prevalent in a society (Chiu et al., 2010; Zou et al., 2009). Intersubjective norms of religiosity, for instance, refer to the shared perception of the importance of religion within a society or culture. Rather than expressing the importance of religious beliefs and behaviors in one's own personal life, intersubjective norms of religiosity (henceforth: cultural norms of religiosity) uncover the perceived importance of religious beliefs and behaviors for the average person within their culture. Religious individuals may be more likely to benefit from being religious when their convictions and behaviors align with perceived cultural norms. For countries in which religion is more trivial or even stigmatized, the relation between religiosity and well-being may be absent or even reversed. Relatedly, in secular countries, religion might be practiced relatively often by minority groups, which has been shown to attenuate the positive association between religious involvement and well-being (Hayward \& Elliott, 2014; Huijts \& Kraaykamp, 2011; May \& Smilde, 2016; Okulicz-Kozaryn, 2010).

\section{A Many-Analysts Approach}

In the current project, we aim to shed light on the association between religion and well-being and the extent to which different theoretically- or methodologically-motivated analytic choices affect the results. To this end, we initiated a many-analysts project, in which several independent analysis teams analyze the same dataset in order to answer a specific research question (e.g., Bastiaansen et al., 2020; Boehm et al., 2018; BotvinikNezer et al., 2020; Silberzahn \& Uhlmann, 2015; van Dongen et al., 2019). A many-analysts approach has been proposed as a way to mitigate the influence of individual-researcher biases (e.g., confirmation bias by the proponent of a theory or disconfirmation bias by the skeptic), especially since the analysis teams are not typically invested in the outcome. More generally, a many-analysts study is arguable less vulnerable to publication bias toward publishing only significant rather than null results, which may lower the (unconscious) tendency toward p- 
hacking by individual analysts. A many-analysts approach can balance out the effects of researcher bias while still allowing for expertise-based analytic decisions such as reasonable preprocessing steps, variable exclusion, and model specification. As such, it enables one to assess the robustness of outcomes and quantify variability based on theory-driven analysis decisions and plausible statistical models. Specifically, we believe that the more consistent the results from different analysis teams are, the more confident we can be in the conclusions we draw from the results. A many-analysts approach may be preferable to an exhaustive multiverse analysis (Steegen et al., 2016) that might simply include the full spectrum of options, including those that are theoretically and methodologically unrealistic.

The idea of inviting different analysis teams to answer the same research question using the same data is relatively novel (Silberzahn and Uhlmann, 2015; see Aczel et al., 2021 for general guidelines); we are aware of three papers in neuroscience (Botvinik-Nezer et al., 2020; Fillard et al., 2011; Maier-Hein et al., 2017), one in microeconomics (HuntingtonKlein et al., 2021), and eight in psychology, three of which pertain to cognitive modeling (Boehm et al., 2018; Dutilh et al., 2019; Starns et al., 2019) while the remaining five are from other fields of psychology (Bastiaansen et al., 2020; Salganik et al., 2020; Schweinsberg et al., 2021; Silberzahn et al., 2018; van Dongen et al., 2019). Most similar to the current work are the projects that applied a many-analysts approach to perform statistical inference on the relation between two variables, such as skin color and red cards in soccer (Silberzahn et al., 2018), scientist gender and verbosity (Schweinsberg et al., 2021), or amygdala activity and stress (van Dongen et al., 2019). While the exact focus of previous many-analysts projects varied (e.g., experience sampling, fMRI preprocessing, predictive modeling, proof of the many-analysts concept), the take-home messages were rather consistent: all papers showed that different yet equally justifiable analytic choices result in very different outcomes, sometimes with statistically significant effects in opposite directions (e.g., Schweinsberg et al., 2021; Silberzahn et al., 2018). In addition, it has proved difficult to pinpoint the exact sources of variability due to the fact that analytic approaches differed in many respects simultaneously (e.g., exclusion criteria, inclusion of covariates etc.). Nev- 
ertheless, the outcomes of these previous projects suggest that choices of statistical model (Silberzahn et al., 2018), statistical framework (van Dongen et al., 2019), (pre)processing software (Botvinik-Nezer et al., 2020), and the variables themselves (Schweinsberg et al., 2021) exert substantial effects on the results and conclusions.

We believe a many-analysts approach is uniquely suited to address various concerns in the study of religion and well-being. First, the relation between religion and health has been researched for decades with hundreds of qualitative reports, cross-sectional and longitudinal studies, and even randomized controlled trials with religious/spiritual interventions for mental health issues (Captari et al., 2018; Harris et al., 2018; Koenig et al., 2020; Rosmarin et al., 2010). Yet new studies keep emerging (e.g., Chang et al., 2021; Luo \& Chen, 2021; Simkin, 2020) and the debate seems far from settled (see for instance the recent special issue in the International Journal for the Psychology of Religion; van Elk, 2021). Second, both 'religion' and 'well-being' are broad and multifaceted constructs that are sensitive to different measures and operationalizations, which might result in both quantitatively and qualitatively different conclusions (Hackney \& Sanders, 2003; Poloma \& Pendleton, 1989). Third, the standard way to assess robustness of an effect or association is often through meta-analysis, but the fragmentation of the literature on the religion-health link and methodological heterogeneity between studies challenge the use and validity of meta-analyses in this domain (Koenig et al., 2021). In general, meta-analyses may suffer from several drawbacks such as publication bias and sensitivity to arbitrary methodological choices (e.g., different meta-analytic techniques can result in different conclusions; de Vrieze, 2018; van Elk et al., 2015). Moreover, the estimated effect sizes in meta-analyses might be as much as three times larger than in preregistered multiple-site replication studies (Kvarven et al., 2020). Fourth, the discussion on the potential health-benefits of religion has been muddied by concerns about researcher interests and biases. That is, it has been argued that scholars of religion might be biased by their own (religious) beliefs (Ladd \& Messick, 2016; Swigart et al., 2020; Wulff, 1998) or by the fact that a substantial amount of research in the science of religion is funded by religiously-oriented organizations such as 
the John Templeton Foundation (Bains, 2011; Wiebe, 2009). ${ }^{1}$ Inviting independent analysts from various backgrounds including but not restricted to religious studies attenuates this potential concern. Moreover, in addition to quantifying variability, with a sufficiently large number of analysis teams one can also investigate factors that might explain observed variability, such as those related to theoretical or methodological expertise and prior beliefs (Aczel et al., 2021). ${ }^{2}$

In addition to the theoretical rationale for using a many-analysts approach to answer the research questions at hand, we also consider the current dataset particularly appropriate for such an approach. That is, the complexity of the data allows for many justifiable choices for the operationalization of the variables and the statistical approach to be employed. While the questions posed to the participants in the cross-cultural study could no longer be changed, the specific method of derivation for the religiosity and well-being scores was at the discretion of the many analysts. At the same time, the research questions and data structure (cross-sectional correlational data) were sufficiently intuitive and manageable to inspire many researchers in the fields of (social) psychology, religious studies, health science, and general methodology to propose an analysis.

Finally, we believe that our project involves a combination of elements that extend existing many-analysts work. First, we collected new data for this project with the aim to provide new evidence for the research questions of interest, as opposed to using an existing dataset that has been analyzed before. Second, we targeted both researchers interested in methodology and open science, as well as researchers from the field of the scientific study of religion and health to encourage both methodologically sound and theoretically relevant decisions (see the section 'Analysis teams'). Third, in comparison to previous many-analysts projects in psychology, the current project includes a lot of teams (i.e., 120 vs. 4, 12, 14, 17, 27, 29, and 70 teams, though note that a machine learning project included 160 analyst

\footnotetext{
${ }^{1}$ Ironically, so is the present project.

${ }^{2}$ Note that we acknowledge that another important problem in the literature on religion and well-being concerns the issue of causality. However, as our project uses non-experimental cross-sectional data, this issue cannot immediately be addressed in the current study (but see Grosz et al., 2020; Rohrer, 2018 for a perspective on causal inference in non-experimental studies).
} 
teams; Salganik et al., 2020). Fourth, we applied a two-step procedure that ensured a purely confirmatory status of the analyses: in stage 1, all teams first either completed a preregistration or specified an analysis pipeline based on a blinded version of the data. After submitting the plan to the OSF, teams received the real data and executed their planned analyses in stage 2 (see Sarafoglou et al., 2022 for more details on and an empirical investigation of preregistration vs. data blinding based on the present data). Fifth, the many-analysts approach itself was preregistered prior to cross-cultural data collection (see osf.io/xg8y5), although the details of the processing and analysis of the many-analysts data were not preregistered.

\section{The Dataset}

The dataset provided to the analysts featured data from 10,535 participants from 24 countries collected in 2019. The data were collected as part of the cross-cultural religious replication project (see also Hoogeveen et al., 2021; Hoogeveen and van Elk, 2018). The dataset contained measures of religiosity, well-being, perceived cultural norms of religion, as well as some demographic items. The full dataset, the data documentation file, and original questionnaire can be found on the OSF project page (osf.io/qbdce/).

Participants. Participants were recruited from university student samples, from personal networks, and from (demographically representative) samples accessed by panel agencies and online platforms (MTurk, Kieskompas, Sojump, TurkPrime, Lancers, Qualtrics panels, Crowdpanel, and Prolific). Participants were compensated for participation by financial remuneration, the possibility for a reward through a raffle, course credits, or received no compensation. Everyone aged 18 years or above could participate. ${ }^{3}$ Participants were required to answer all multiple choice questions, and hence there were no missing data (except for 36 people who did not provide a numeric age and 995 people who chose not to answer the item on sexual satisfaction, as this was the only item for which participants were not required to provide an answer.) The countries were convenience-sampled (i.e.,

\footnotetext{
${ }^{3}$ Note that we did not exclude the 19 participants who indicated they were younger than 18 (but some of the analysis teams did exclude these participants).
} 
through personal networks), but were selected to cover six continents and include different ethnic and religious majorities. The final sample included individuals who identified as Christian (31.2\%), Muslim (6.1\%), Hindu (2.9\%), Buddhist (2.0\%), Jewish (1.0\%), or were part of another religious group (2.9\%). Finally, 53.9\% of participants did not identify with any religion. See Tables B1 and B2 in Appendix B for the full descriptive statistics of the dataset.

Measures. Personal religiosity was measured using nine standardized self-report items taken from the World Values Survey (WVS; World Values Survey, 2010), covering religious behaviors (institutionalized such as church attendance and private such as prayer/meditation), beliefs, identification, values, and denomination. The well-being measure consisted of 18 self-report items from the validated short version of Quality of Life scale, as used by the World Health Organization (WHOQOL-BREF; WHOQOL Group, 1998). Included items cover general health and well-being, as well as the domains of physical health, psychological health and social relationships. Specific items evaluated: the quality of life in general, and satisfaction of overall health (general); pain, energy, sleep, mobility, activities, dependence on medication, and work capability (physical domain); life enjoyment, concentration, self-esteem, body-image, negative feelings, and meaningfulness (psychological domain); as well as personal relationships, social support, and sexual satisfaction (social domain). In addition to the raw scores for each item, we also provided an overall mean, as well as three means per subscale, following the calculation instructions in the WHOQOL-BREF manual. Cultural norms of religiosity were measured with two items assessing participants' perception of the extent to which the average person in their country considers a religious lifestyle and belief in God/Gods/spirits important (Wan et al., 2007). Finally, demographics were measured at the individual level (i.e., age, gender, level of education, subjective socioeconomic status (SES), and ethnicity) whereas GDP per capita (current US\$, World Bank Group, 2017), sample type (e.g., university students, online panels), and means of compensation (e.g., course credit, monetary reward) were determined at the country/sample level. Items were reverse-coded when applicable. Personal religiosity 
items were additionally rescaled to the $0-1$ range to make them contribute equally to an average religiosity score since the items were measured on different scales (e.g., a 1-8 Likert scale or a 'yes/no' item, which was coded as 'no' $=0$ and 'yes' $=1) .{ }^{4}$ GDP was provided as a raw value as well as standardized at the country level.

\section{Disclosures}

\section{Data, materials, and preregistration}

At the start of this project we did not envision a particular statistical analysis to be executed across the reported results from the individual teams, and therefore we did not preregister any statistical inference procedure. However, at an earlier stage, we did preregister our own hypotheses regarding the research questions that were posed to the analysis teams (see osf.io/zyu8c/). This preregistration also anticipates the many-analysts approach, yet does not specify the exact details of the project. In this preregistration document, we indicated that the analysis teams would first receive a blinded version of the data, but we later decided that half of the teams would work with blinded data and the other half would write their own preregistration (see Sarafoglou et al., 2022). Note that we did not include our own estimated effect sizes in the results as shown below. Our results, however, do corroborate the overall pattern of results from the analysis teams. Interested readers can access our preregistered analysis of the research questions on the OSF (osf.io/vy8z7/).

All documents provided to the analysis teams (dataset, documentation, questionnaire), as well as the administered surveys, the anonymized raw and processed data (including relevant documentation), and the $\mathrm{R}$ code to conduct all analyses (including all figures), can be found on the project page on the OSF (osf.io/vy8z7/). Identifying information (such as names, email-addresses, universities) was removed from all free-text answers. See also Table 2 for an overview of all resources.

\footnotetext{
${ }^{4}$ When teams indicated that they preferred the raw data, we provided the function to back-transform the data.
} 


\section{Reporting}

We report how we determined our sample size, all data exclusions, and all manipulations in the study. However, it should be noted since this project also involved an empirical evaluation of analysis blinding, which is reported in another paper (i.e., Sarafoglou et al., 2022). Here, we only describe measures relevant to the theoretical research questions and the many-analysts approach. The description of the remaining measures that were only used for the experimental analysis proposal manipulation can be found in Sarafoglou et al. (2022).

\section{Ethical approval}

The study was approved by the local ethics board of the University of Amsterdam (registration number: 2019-PML-12707). All participants were treated in accordance with the Declaration of Helsinki. See Appendix B for details on the ethical approval for the cross-cultural data collection.

\section{Methods}

\section{Analysis Teams}

The analysis teams were recruited through advertisements in various newsletters and email lists (e.g., the International Association for the Psychology of Religion (IAPR), International Association for the Cognitive Science of Religion (IACSR), Society for Personality and Social Psychology (SPSP), and the Society for the Psychology of Religion and Spirituality (Div. 36 of the APA)), on social media platforms (i.e., blogposts and Twitter), and through the authors' personal network. We invited researchers of all career stages (i.e., from doctoral student to full professor). Teams were allowed to include graduate and undergraduate students in their teams as long as each team also included a $\mathrm{PhD}$ candidate or a more senior researcher. Initially, $N=173$ teams signed up to participate in the manyanalysts project. From those teams, $N=127$ submitted an analysis plan and $N=120$ 


\section{Table 1}

Career Stages and Domains of Expertise Featured in the 120 Analysis Teams.

\begin{tabular}{ll}
\hline & Percentage of teams \\
\hline Career Stages & \\
Doctoral Student & $54(45 \%)$ \\
Post-doc & $45(37.50 \%)$ \\
Assistant Professor & $32(26.67 \%)$ \\
Associate Professor & $26(21.67 \%)$ \\
Full Professor & $20(16.67 \%)$ \\
Domains of Expertise & \\
Social Psychology & $43(35.83 \%)$ \\
Cognition & $28(23.33 \%)$ \\
Methodology and Statistics & $25(20.83 \%)$ \\
Religion and Culture & $25(20.83 \%)$ \\
Psychology (Other) & $19(15.83 \%)$ \\
Health & $17(14.17 \%)$ \\
\hline
\end{tabular}

Note. Teams may include multiple members of the same position and in the same domain.

completed the project. The members from each analysis team were offered co-authorship on the main manuscript. No individual researcher or team was excluded from the study.

The number of analysts per team ranged from 1 to 7 , with most teams consisting of $1(41 \%)$ or $2(33 \%)$ analysts (median $=2)$. The different career stages and domains of expertise featured in the analysis teams are given in Table 1. In addition, Figure 1 shows the self-rated collective knowledge about the topic of religion and well-being and about methodology and statistics. As becomes evident, most of the analysis teams had more methodological and/or statistical expertise than substantive expertise; $80 \%$ of the teams reported considerable expertise with regard to methods and statistics compared to $31 \%$ with regard to religion and well-being, $19 \%$ compared to $17 \%$ was neutral, and $3 \%$ compared to $50 \%$ reported little to no knowledge, respectively. 


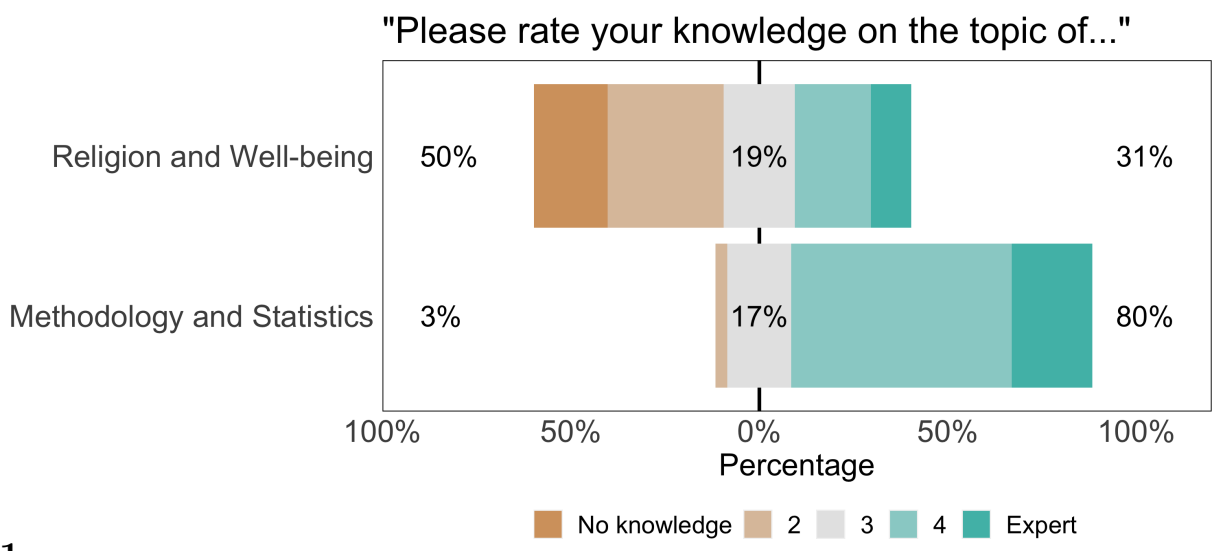

\section{Figure 1}

Responses to the survey questions on self-rated topical and methodological knowledge. The top bar represents the teams' answers about their knowledge regarding religion and well-being and the bottom bar represents the teams' answers about their knowledge regarding methodology and statistics. For each item, the number to the left of the data bar (in brown/orange) indicates the percentage of teams that reported little to no knowledge. The number in the center of the data bar (in grey) indicates the percentage of teams that were neutral. The number to the right of the data bar (in green/blue) indicates the percentage of teams that reported (some) expertise.

\section{Sampling Plan}

For a separate component of the project (see Sarafoglou et al., 2022), the preregistered sample size target was set to a minimum of 20 participating teams, which was based on the recruited analysis teams in the many-analysts project from Silberzahn et al. (2018). However, we did not set a maximum number of participating teams. The recruitment of analysis teams was ended on December 22, 2020.

\section{Materials}

\section{Surveys}

The analysts received three surveys, here referred to as the pre-survey, the midsurvey, and the post-survey. In the pre-survey, participating teams indicated the career stages and domains of expertise featured in their team, self-rated their (collective) theoretical and methodological knowledge (self-reported; 5-pt Likert scale), and anticipated the likelihood of the effects of interest (7-pt Likert scale). In the mid-survey, teams were asked 
about the experienced effort, frustration, workload in hours spent on the project, and the extent to which this workload was lower or higher than expected for the analysis planning phase (i.e., stage 1; 7-pt Likert scales). In the post-survey, the teams provided the results of their analyses and again indicated their experiences during the analysis executing phase (i.e., stage 2). Specifically, per research question, teams were asked about their statistical approach, the operationalization of the independent variable(s) and dependent variable(s), included covariates, analytic sample size, (unit of) effect size, $p$-value or Bayes factor, and additional steps they took for the analysis. Furthermore, for both research questions, the teams gave a subjective conclusion about the evidence for the effect (i.e., "good evidence for a relation", "ambiguous evidence", or "good evidence against a relation"), about the practical meaningfulness/relevance of the effect (based on the data; "yes" or "no"), and indicated again the likelihood of the effects of interest (on a 7-pt Likert scale). Additionally, teams indicated the appropriateness of their statistical approach (7-pt Likert scale), the suitability of the dataset for answering each research question (7-pt Likert scale), and whether or not they deviated from their planned analysis. In case this last question was answered affirmatively, they specified with regard to which aspects they deviated (i.e., hypotheses, included variables, operationalization of the independent variable(s), operationalization of the dependent variable(s), exclusion criteria, statistical test, statistical model, direction of the effect). Finally, teams again reported the experienced effort, frustration, workload in hours and the extent to which this workload was lower or higher than expected for stage 2 (on 7-pt Likert scales).

\section{Procedure}

After signing up, participating teams received a document outlining the aim of the project, the timeline, a short theoretical background with respect to the research questions, and a description of the dataset. Then, after completing the pre-survey, teams could access the full data documentation, the questionnaire as presented to the participants of the cross-cultural study, and either a blinded version of the data or a preregistration template, 


\section{Table 2}

Overview of Project Stages and Resources.

\begin{tabular}{ll}
\hline Process & Link \\
\hline Stage 1 & \\
Recruitment and sign-up & osf.io/hpd6b \\
Pre-survey & osf.io/kgqze \\
Access to data documentation, questionnaire and either of: & \\
a) preregistration form & osf.io/a5ent \\
b) blinded data & osf.io/ktvqw \\
Design analysis and upload plan & OSF team pages \\
Mid-survey & osf.io/kgqze \\
Stage 2 & \\
Access to data & osf.io/6njsy \\
Execute analysis and upload script (optional: + report) & OSF team pages \\
Post-survey & osf.io/kgqze \\
Lead team: summarize and write-up key results & \\
Invite analysis teams to write commentary & \\
\hline
\end{tabular}

Note. See osf.io/vy8z7 for an overview of all team pages.

depending on which condition they had been assigned to. Teams could then design their analysis and upload their documents on their own team page on the OSF (deadline: December 22nd, 2020). The project leaders 'froze' the stage 1 documents and sent the link to the mid-survey. Upon completion of this survey, teams automatically received access to the real data. They could execute and upload their final analysis scripts on the OSF until February 28th, 2021. Teams were encouraged to also upload a document summarizing their results, but this was not mandatory. Finally, all teams completed the post-survey. See Table 2 for an overview of the procedure.

\section{Results}

Here, we report the key results of the project. Specifically, we evaluate the teams' reported effect sizes and their subjective conclusions about the research questions (i.e., the primary results). In addition, we provide descriptive results about the many-analysts aspect (i.e., the secondary results: variability in analytic approaches, included variables, and the 
teams' experiences across the two different stages). Finally, we assessed whether or not the reported effect sizes are related to subjective beliefs about the likelihood of the research questions.

\section{Primary Results}

Teams could report any effect size metric of their choosing, but we noted that we preferred a beta coefficient (i.e., a fully standardized coefficient; z-scored predictors and outcomes) to allow for a comparison between teams. As we correctly anticipated that (1) most teams would conduct linear regression analyses (see Table 3) and (2) both the (scale of the) independent and dependent variables might vary across teams, we considered a beta coefficient the most suitable effect size metric. Note that our request for beta coefficients as effect size metrics may have affected the teams' choice of statistical model and encouraged them to use regression models that generate beta coefficients. For teams that did not provide

a (fully) standardized coefficient, we recalculated the beta based on the respective team's analysis script whenever possible. Specifically, for (multilevel) linear regression models we used the effectsize package or the jtools package to extract standardized coefficients in R. For analyses in SPSS and non-standard models in R, we standardized the data manually prior to executing the analyses. Finally, many teams reported multiple effect sizes, as they either separately considered multiple predictors (e.g., religious beliefs and religious behaviors) and/or multiple dependent variables (e.g., psychological well-being and physical well-being). In that case, we asked the teams to provide us with one primary effect size they considered most relevant to answer the research question or to select one randomly. In the Online Appendix, we additionally list (1) effect sizes for the different subscales of the well-being measure as reported by the teams and (2) effect sizes from teams that could not provide a beta coefficient (e.g., machine learning models). 


\section{Research Question 1: "Do religious people self-report higher well-being?"}

We were able to extract 99 beta coefficients from the results provided by the 120 teams that completed stage $2 .{ }^{5}$ As shown in Figure 2, the results are remarkably consistent: all 99 teams reported a positive beta value, and for all teams the $95 \%$ confidence/credible interval excludes zero. The median reported beta is 0.120 and the median absolute deviation is 0.036 . Furthermore, $88 \%$ of the teams concluded that there is good evidence for a positive relation between religiosity and self-reported well-being. Notably, although the teams were almost unanimous in their evaluation of research question 1, only eight of the 99 teams reported combinations of effect sizes and confidence/credibility intervals that matched those from another team (i.e., four effect sizes were reported twice). Do note that in contrast to the unanimity in results based on the beta coefficients, out of the 21 teams for whom a beta coefficient could not be calculated, 3 teams reported evidence against the relation between religiosity and well-being: 2 teams used machine learning and found that none of the religiosity items contributed substantially to predicting well-being and 1 team used multilevel modeling and reported unstandardized gamma-weights for withinand between-country effects of religiosity whose confidence intervals included zero (see the Online Appendix).

Figure 3 displays the average prior and final beliefs about the likelihood of the hypothesis. Researchers' prior beliefs about religiosity being positively related to self-reported well-being were already high ( $M=4.90$ on the 7-point Likert scale), but were raised further after them having conducted the analysis ( $M=5.49$ on the 7 -point Likert scale). Specifically, before seeing the data, $72 \%$ of the teams considered it likely that religiosity is related to higher self-reported well-being. This percentage increased to $85 \%$ after having seen the data, while $11 \%$ were neutral and $3 \%$ considered it unlikely. Finally, $75 \%$ of teams indicated the relation to be relevant or meaningful based on these data.

\footnotetext{
${ }^{5}$ One team misinterpreted the scoring of the items and hence miscoded the direction of the effect. As they subsequently also based their subjective conclusions on the incorrect results, we excluded the reported effect sizes, subjective evaluation, and prior+final beliefs about the likelihood of the hypotheses for this team.
} 


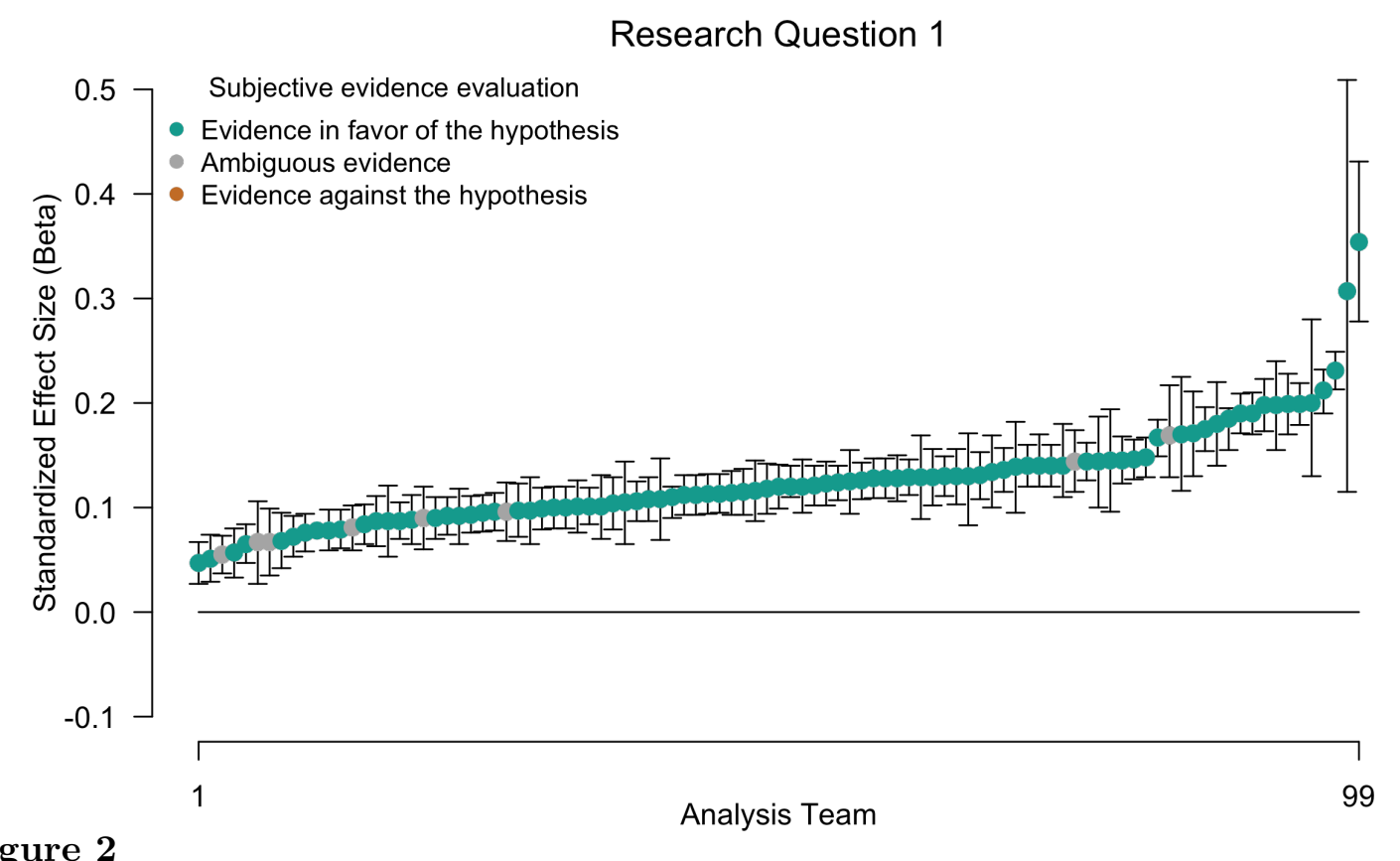

\section{Figure 2}

Beta coefficients for the effect of religiosity on self-reported well-being (research question 1) with 95\% confidence or credible intervals. Green/blue points indicate effect sizes of teams that subjectively concluded that there is good evidence for a positive relation between individual religiosity and self-reported well-being, grey points indicate effect sizes of teams that subjectively concluded that the evidence is ambiguous, and brown/orange points indicate effect sizes of teams that subjectively concluded that there is good evidence against a positive relation between individual religiosity and self-reported well-being. The betas are ordered from smallest to largest.

\section{Research Question 2: "Does the relation between religiosity and self-reported well-being depend on perceived cultural norms of religion?"}

Out of the 120 teams who completed stage 2 we were able to extract 101 beta coefficients for research question 2. As shown in Figure 4 the results for research question 2 are more variable than for research question 1; 97 out of 101 teams reported a positive beta value and for 66 teams $(65 \%)$ the confidence/credible interval excluded zero. The median reported effect size is 0.039 and the median absolute deviation is 0.022 . Furthermore, $54 \%$ of the teams concluded that there is good evidence for an effect of cultural norms on the relation between religiosity and self-reported well-being. Again, most reported effect sizes were unique; only 3 out of the 101 reported combination of effect size and confidence/credible 


\section{"How likely do you think it is that religiosity is related to higher self-reported well-being?"}

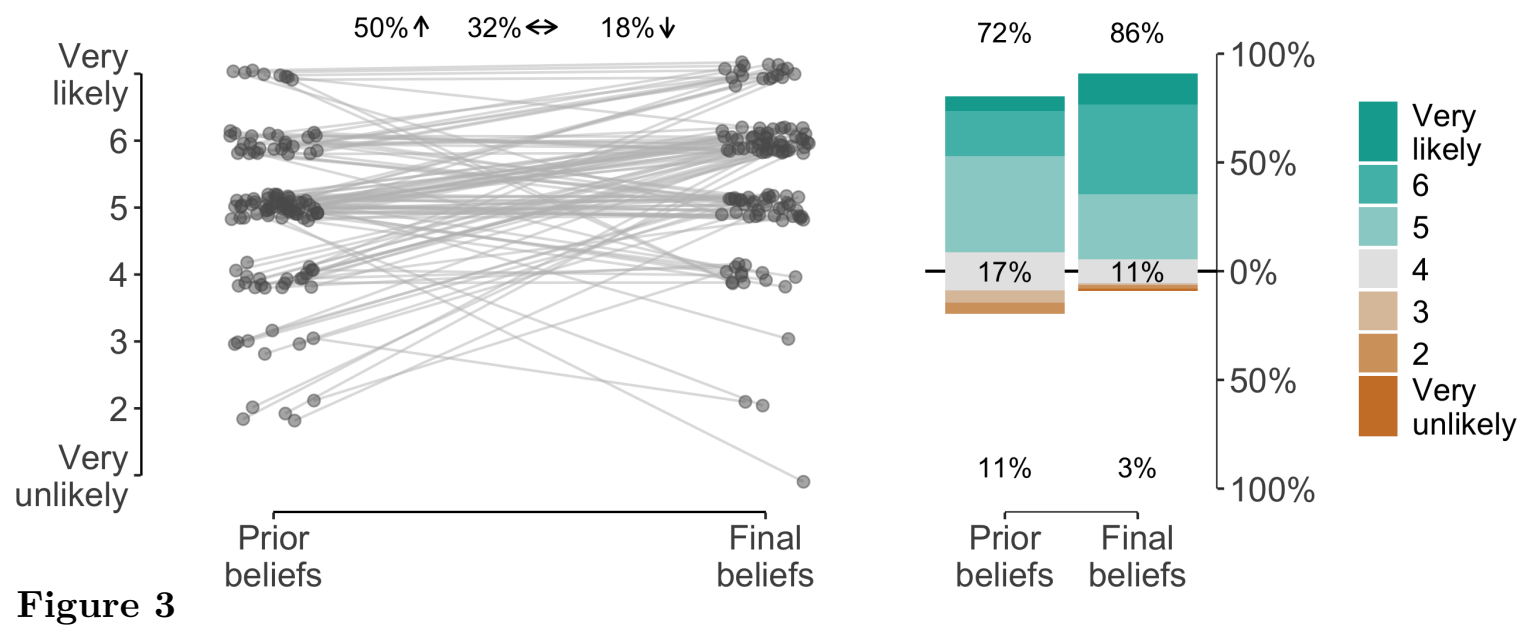

Responses to the survey questions about the likelihood of hypothesis 1 . The left side of the figure shows the change in beliefs for each analysis team. Fifty percent of the teams considered the hypothesis somewhat more likely after having analyzed the data than prior to seeing the data, 18\% considered the hypothesis less likely after having analyzed the data, and 32\% did not change their beliefs. Likelihood was measured on a 7-point Likert scale ranging from 'very unlikely' to 'very likely'. Points are jittered to enhance visibility. The right side of the figure shows the distribution of the Likert response options before and after having conducted the analyses. The number at the top of the data bar (in green/blue) indicates the percentage of teams that considered the hypothesis (very) likely, the number in the center of the data bar (in grey) indicates the percentage of teams that were neutral, and the number at the bottom of the data bar (in brown/orange) indicates the percentage of teams that considered the hypothesis (very) unlikely.

intervals appeared twice.

Figure 5 shows the researchers' average prior and final beliefs about the likelihood of the second hypothesis. As for research question 1, prior beliefs about the hypothesis were rather high. However, in contrast to research question 1, conducting the analysis lowered beliefs about the likelihood of hypothesis 2. Specifically, before seeing the data, $71 \%$ of the teams considered it likely that the relation between religiosity and self-reported wellbeing depends on perceived cultural norms of religion. This percentage dropped to $54 \%$ after having seen the data, while $19 \%$ were neutral and $27 \%$ considered it unlikely. Finally, only about half of the teams (49\%) indicated the effect of cultural norms to be relevant or meaningful based on these data. 


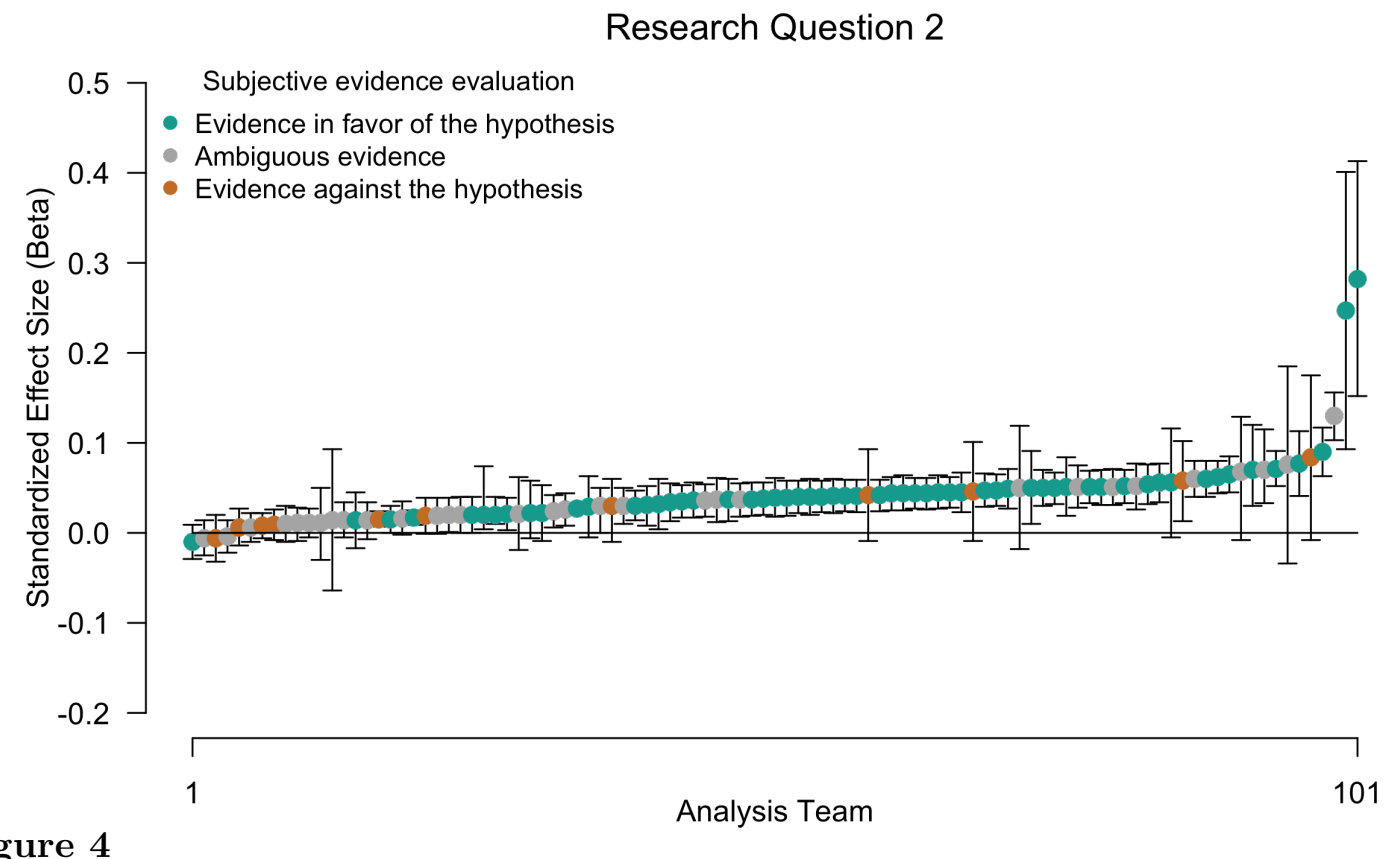

\section{Figure 4}

Beta coefficients for the effect of cultural norms of the relation between religiosity and self-reported well-being (research question 2) with 95\% confidence or credible intervals. Green/blue points indicate effect sizes of teams that subjectively concluded that there is good evidence for the hypothesis that the relation between individual religiosity and self-reported well-being depends on the perceived cultural norms of religion, grey points indicate effect sizes of teams that subjectively concluded that the evidence is ambiguous, and brown/orange points indicate effect sizes of teams that subjectively concluded that there is good evidence against the hypothesis that the relation between individual religiosity and self-reported well-being depends on the perceived cultural norms of religion. The betas are ordered from smallest to largest.

\section{Secondary Results}

In addition to evaluating the overall results for the two main research questions, we also assessed perceived suitability of the data and analytic approaches, variability in analytical approaches (i.e., statistical models), variable inclusion, and teams' experiences during the two stages of the project.

\section{Perceived Suitability of Dataset}

At the end of the project, all teams reported how suitable they found the current dataset for answering the research questions. As shown at the top of Figure 6, most teams considered the data (very) suitable for answering the research questions: for research ques- 


\section{"Does the relation between religiosity and self-reported well-being depend on perceived cultural norms of religion?"}
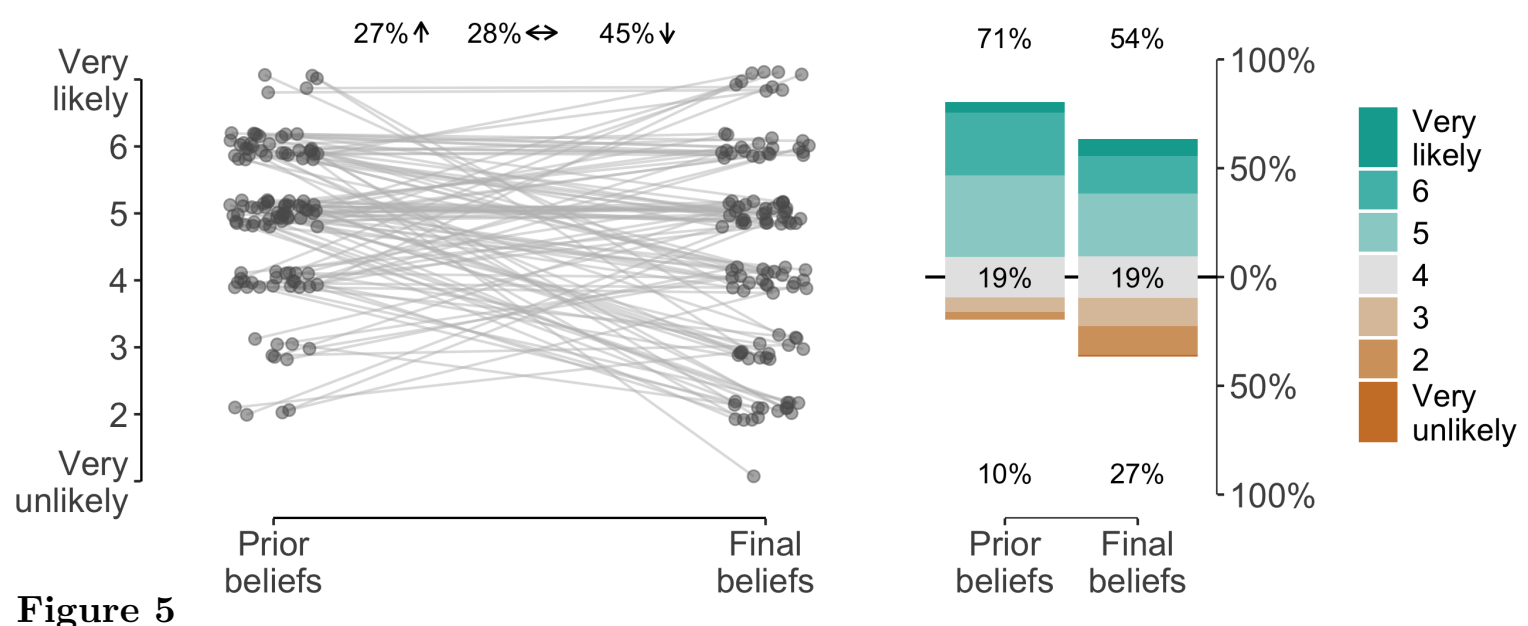

Responses to the survey questions about the likelihood of hypothesis 2. The left side of the figure shows the change in beliefs for each analysis team. Twenty-seven percent of the teams considered the hypothesis somewhat more likely after having analyzed the data than prior to seeing the data, $45 \%$ considered the hypothesis less likely having analyzed the data, and 28\% did not change their beliefs. Likelihood was measured on a 7-point Likert scale ranging from 'very unlikely' to 'very likely'. Points are jittered to enhance visibility. The right side of the figure shows the distribution of the Likert response options before and after having conducted the analyses. The number at the top of the data bar indicates the percentage of teams that considered the hypothesis (very) likely, the number in the center of the data bar (in grey) indicates the percentage of teams that were neutral, and the number at the bottom of the data bar (in brown/orange) indicates the percentage of teams that considered the hypothesis (very) unlikely.

tion 1, $86 \%$ found the data suitable, $8 \%$ neutral, and $6 \%$ unsuitable; for research question 2, $70 \%$ found the data suitable, $19 \%$ neutral, and $11 \%$ unsuitable.

\section{Analytic Approaches}

Table 3 displays the different statistical approaches used in the project, as well as the percentage of teams that employed the respective approach. While a total of 25 different statistical methods was mentioned, (multilevel) linear regression was clearly the dominant approach. Specifically, 34\% of the teams used linear regression, another $45 \%$ used multilevel linear regression, and the remaining $21 \%$ used a different approach.

In general, teams were confident that their chosen statistical approach was appro- 
Table 3

Analytic Approaches Taken by the Analysis Teams.

\begin{tabular}{ll}
\hline Analytic Approach & Percentage of teams \\
\hline Multilevel Linear Regression & $45 / 128(35.16 \%)$ \\
Linear Regression & $36 / 128(28.12 \%)$ \\
Bayesian Multilevel Linear Regression & $7 / 128(5.47 \%)$ \\
Structural Equation Model & $6 / 128(4.69 \%)$ \\
ANOVA & $5 / 128(3.91 \%)$ \\
T-test & $4 / 128(3.12 \%)$ \\
Bayesian Linear Regression & $3 / 128(2.34 \%)$ \\
Path Analysis & $3 / 128(2.34 \%)$ \\
Bayesian Multilevel Ordinal Regression & $2 / 128(1.56 \%)$ \\
Ordinal Logistic Regression & $2 / 128(1.56 \%)$ \\
ANCOVA & $1 / 128(0.78 \%)$ \\
Bayesian Additive Regression Trees & $1 / 128(0.78 \%)$ \\
Bayesian ANOVA & $1 / 128(0.78 \%)$ \\
Bayesian Multilevel Structural Equation Model & $1 / 128(0.78 \%)$ \\
Correlation & $1 / 128(0.78 \%)$ \\
Machine Learning & $1 / 128(0.78 \%)$ \\
Meta-Analysis & $1 / 128(0.78 \%)$ \\
Mixed-Effects ANOVA & $1 / 128(0.78 \%)$ \\
Moderated Generalized Linear Regression & $1 / 128(0.78 \%)$ \\
Multilevel Structural Equation Model & $1 / 128(0.78 \%)$ \\
Multiverse Analysis & $1 / 128(0.78 \%)$ \\
Multiverse Of Multilevel Linear Regression & $1 / 128(0.78 \%)$ \\
Network Analysis & $1 / 128(0.78 \%)$ \\
Non-linear Regression & $1 / 128(0.78 \%)$ \\
Non-parametric Partial Correlation & $1 / 128(0.78 \%)$ \\
\hline
\end{tabular}

Note. Some teams reported multiple statistical approaches. 


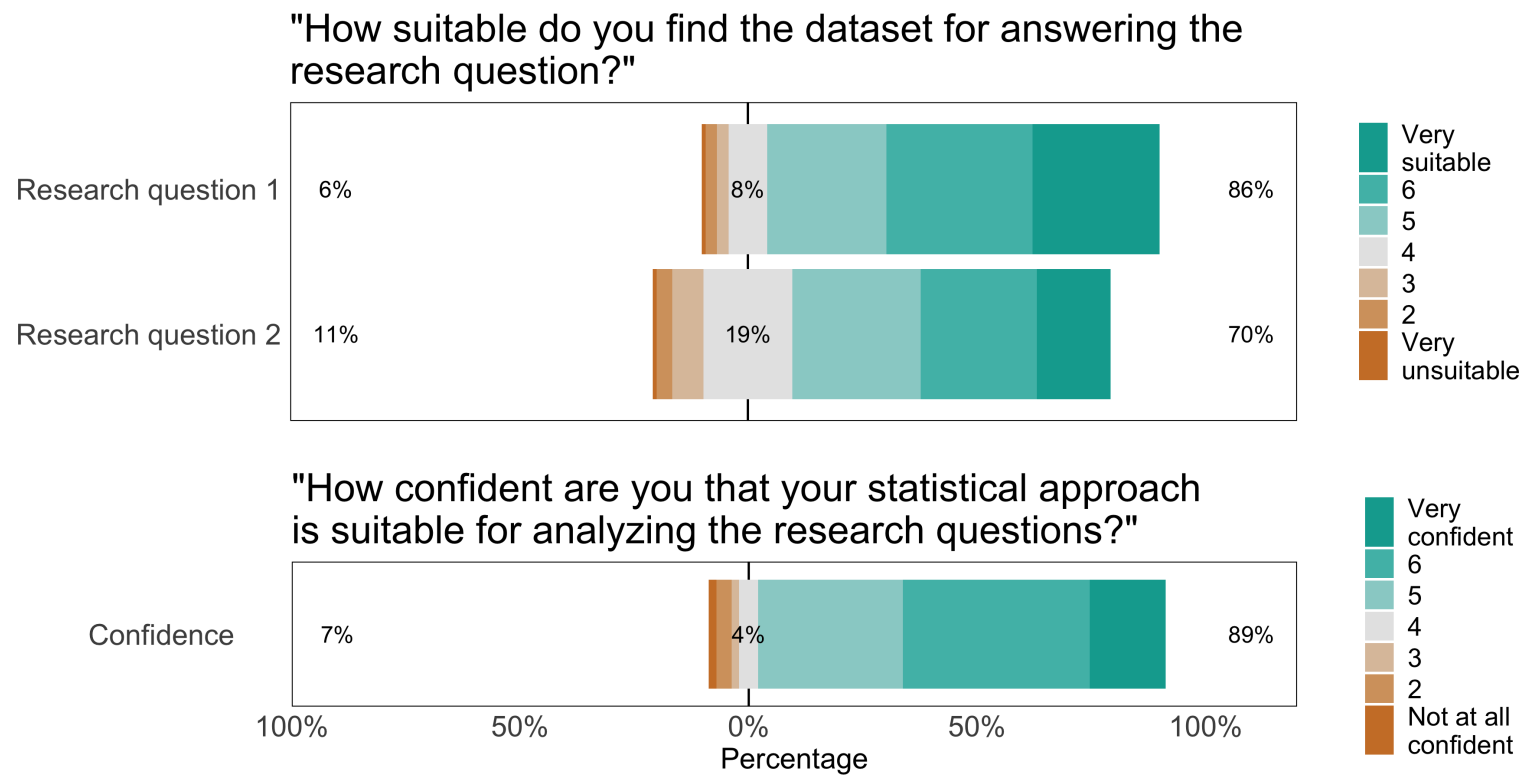

Figure 6

Responses to the survey questions about the suitability of the dataset for answering the research questions (top) and the teams' confidence in their analytic approach (bottom). For question 1, the top bar represents the teams' answers with respect to research question 1 and the bottom bar represents the teams' answers for research question 2. For each item, the number to the left of the data bar (in brown/orange) indicates the percentage of teams that considered the data (very) unsuitable / were not (at all) confident in their approach. The number in the center of the data bar (in grey) indicates the percentage of teams that were neutral. The number to the right of the data bar (in green/blue) indicates the percentage of teams that considered the data (very) suitable / were (very) confident in their approach.

priate for analyzing the research questions; as shown at the bottom of Figure 6, $89 \%$ of the teams indicated to be (very) confident, $4 \%$ was neutral, and $7 \%$ was not (at all) confident. ${ }^{6}$

\section{Variable Inclusion}

For each team we coded which of the items provided in the dataset were included as (1) dependent variable, (2) independent variable, and (3) covariates in the analysis for each research question. ${ }^{7}$

\footnotetext{
${ }^{6}$ Note that out of the 8 teams reporting not being confident, 2 did not submit a final analysis and 2 did not provide a usable effect size.

${ }^{7}$ Please see the document 'variable mapping' on the OSF (osf.io/qbdce/) for how the items correspond to the item names in the datafile.
} 
Dependent Variable. The subjective well-being measure consisted of three subscales (psychological, physical, social), as well as two general items. In the dataset, we provided responses for all 18 individual items as well as an overall mean and one mean for each of the three subscales. Teams could decide to either use any of the provided averages or combine specific items themselves (e.g., take the mean, median, sum). In addition, some teams conducted a factor analysis and used one or multiple extracted factors as the dependent variable. In this case, we coded which items were used as input for the factor analysis. Figure 7 shows the included items as dependent variable aggregated over all teams for research question 1 and research question 2. For research question 1, the most frequently used items are enjoying life and meaningfulness (included by over $43 \%$ of the teams). Note that all but four teams used the same dependent variable for research question 1 and $2 .{ }^{8}$ In Appendix A, we show the included items separately for each team.

Independent Variable. The religiosity measure consisted of 9 primary items on response scales ranging from dichotomous to 8-points and the cultural norms of religiosity measure consisted of two items on a 5-point scale. Averages were not provided in the dataset, but could be created by the teams themselves. Figure 8 shows the included items as independent variable aggregated over all teams for research question 1 and research question 2. In Appendix A, we show the included items separately for each team.

For research question 1 (i.e., the relation between religiosity and self-reported wellbeing), over $75 \%$ of the teams operationalized the independent variable by including the items frequency of service attendance, belief in God/Gods, frequency of prayer, belief in afterlife, personal importance of a religious lifestyle, or personal importance of belief in God. The remaining three religiosity items were used less frequently: $70 \%$ of the teams included the item religious status (religious/not religious/atheist) and spirituality, while only $50 \%$ included religious membership.

For research question 2 (i.e., the effect of perceived cultural norms on the relation between religiosity and self-reported well-being), all but four teams used the interaction

\footnotetext{
${ }^{8}$ Two of the four teams that did not use the same dependent variable for research question 1 and 2 only conducted an analysis for research question 1.
} 


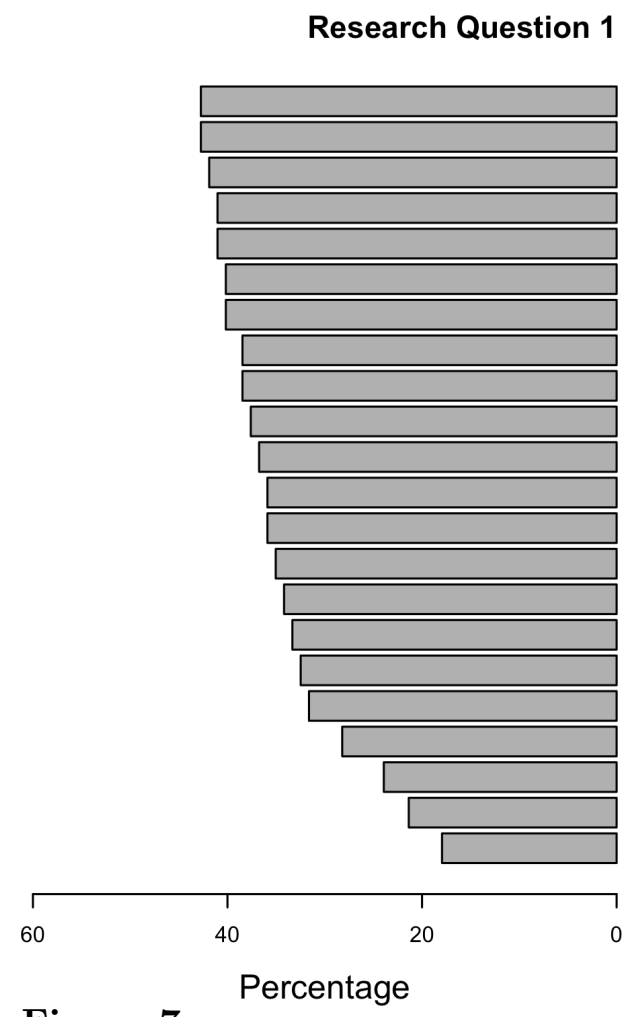

Research Question 2
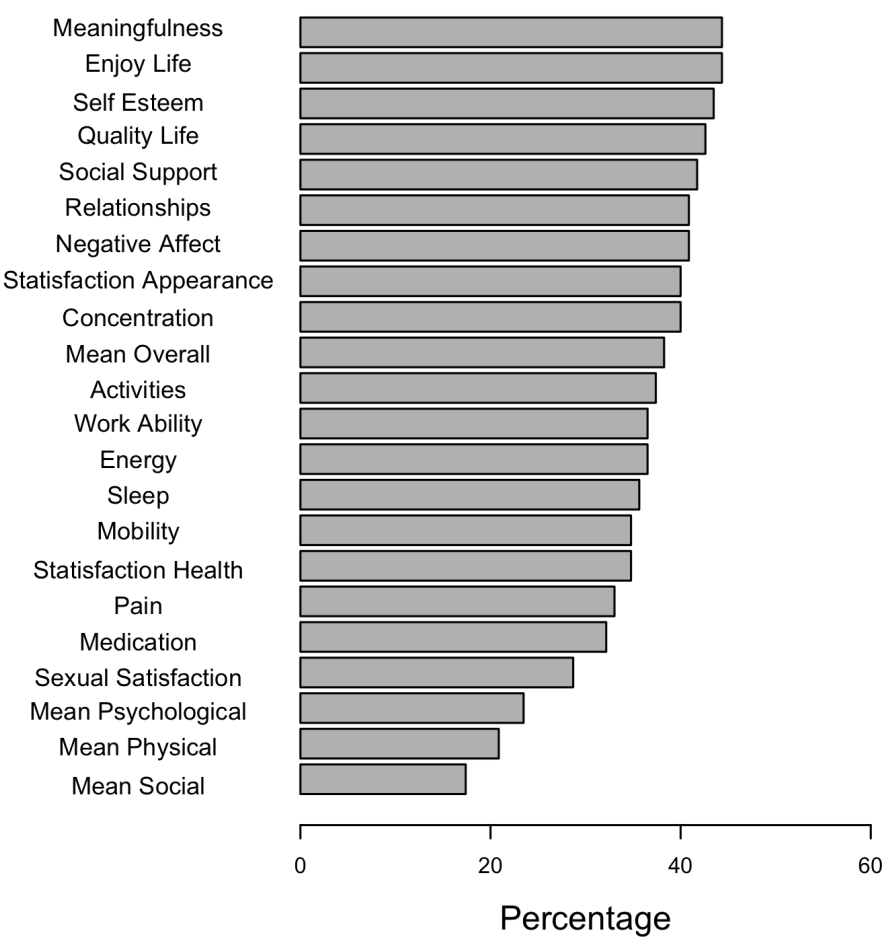

Items included as dependent variables for research question 1 (on the left) and research question 2 (on the right). Note that the averages for the well-being subscales ('Mean Psychological', 'Mean Social', 'Mean Physical'), as well as the overall average ('Mean Overall') were provided by the MARP team.

term between their chosen religiosity measure and their chosen cultural norms measure as the independent variable. ${ }^{9}$ More teams operationalized cultural norms using the item importance of a religious lifestyle in their country (93\%) than importance of belief in God/Gods in their country (89\%). Here again, over $75 \%$ of the teams operationalized the independent variable by including the items frequency of service attendance, belief in God, frequency of prayer, belief in afterlife, personal importance of a religious lifestyle, or personal importance of belief in God, whereas the items religious status (religious/not religious/atheist) and spirituality were included by about $70 \%$ and $68 \%$ of the teams, respectively; only $52 \%$ of the teams included religious membership. Note that almost all teams used the same religiosity

\footnotetext{
${ }^{9}$ The four teams that did not use an interaction in their evaluation of research question 2 either used the main effect of cultural norms on well-being or the main effect of religiosity on well-being (while controlling for cultural norms).
} 


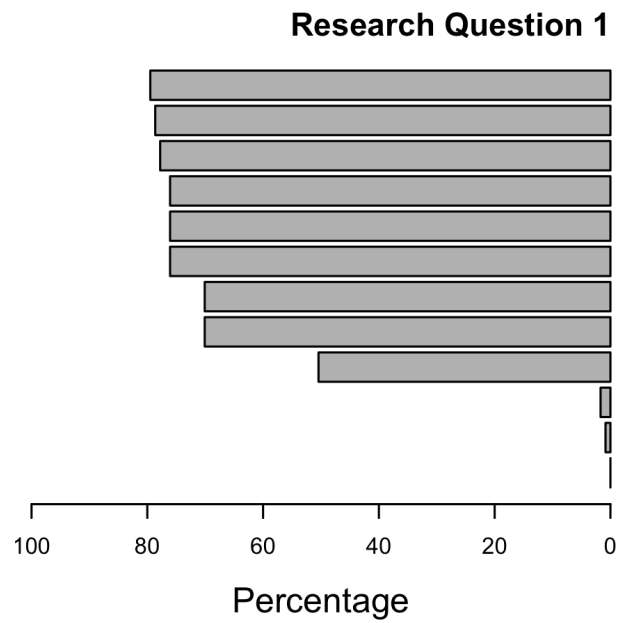

\section{Research Question 2}
Service Attendance Belief God
Prayer
Norms God Self
Norms Lifestyle Self
Belief Afterlife
Spirituality
Religious Status
Membership Norms Lifestyle Country Norms God Country
External Norms

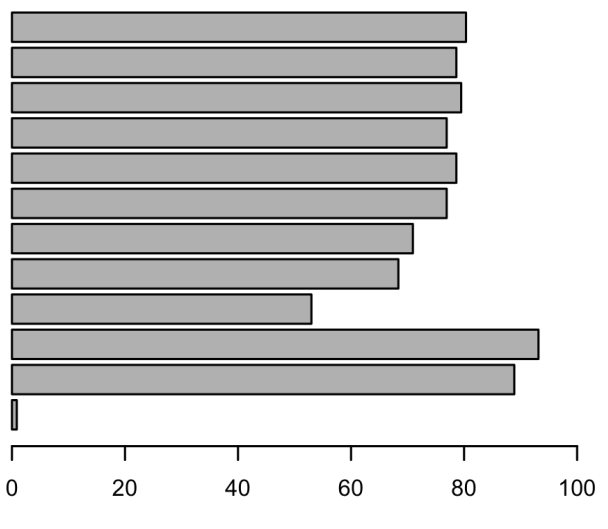

Percentage

Figure 8

Items included as independent variables for research question 1 (on the left) and research question 2 (on the right).

measure for research question 1 and research question 2 .

Covariates. Teams were free to include as covariates in their models any of the measured demographic variables (e.g., age, socio-economic status), country-level variables (e.g., gross domestic product - GDP) or sample characteristics (e.g., general public or student sample, means of compensation). Figure 9 displays the included items as covariates aggregated over all teams for research question 1 and research question 2 . The most frequently included covariates are age (59\%), socio-economic status (55\%), gender (53\%), and education (50\%). Note that per team the choice of covariates was largely equal across research questions, with the exception that the cultural norms items were occasionally added as covariates for research question 1 while they were part of the independent variable for research question 2 .

\section{Teams' Experiences}

Although most teams indicated that effort was (very) high, the majority also reported that frustration was (very) low and that they spent as much time as anticipated (see Figure 10). That is, in stage 1, 55\% of the teams reported (very) high effort, $17 \%$ were neutral, and $28 \%$ reported (very) low effort. For stage 2, $48 \%$ of the teams reported 
Research Question 1

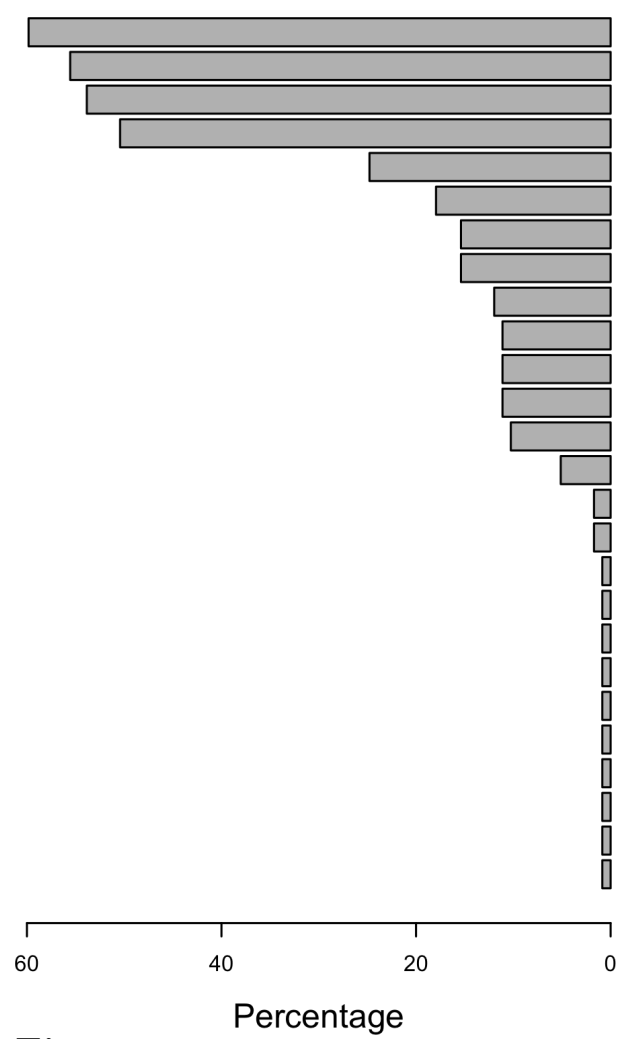

Research Question 2

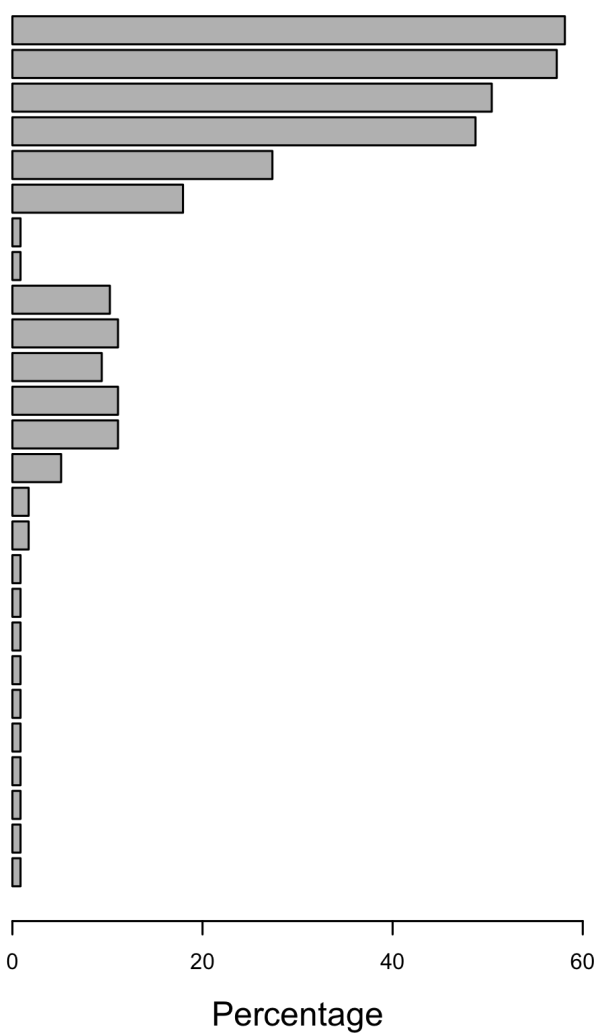

\section{Figure 9}

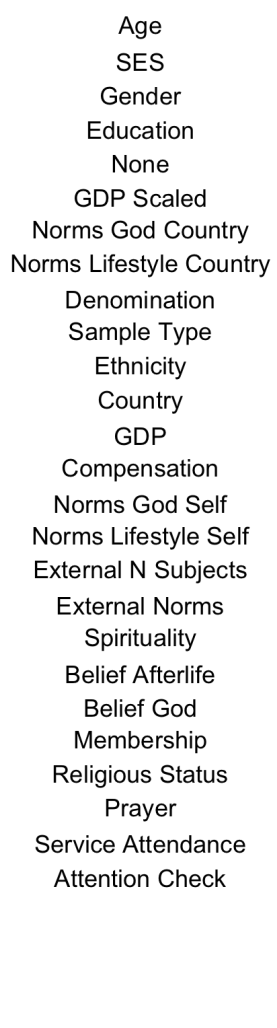

Items included as covariates for research question 1 (on the left) and research question 2 (on the right). Variables indicated as 'external' refer to covariates that are based on data not provided by the MARP team.

(very) high effort, $18 \%$ were neutral, and $34 \%$ reported (very) low effort. In stage 1, $17 \%$ of the teams reported (very) high frustration, $23 \%$ were neutral, and $60 \%$ reported (very) low frustration. In stage $2,18 \%$ of the teams reported (very) high frustration, $17 \%$ were neutral, and $65 \%$ reported (very) low frustration. The median time spent on the analyses was 8 hours for both stages, although the range was quite wide: 1 to 80 hours for stage 1 and 30 minutes to 140 hours for stage 2. Most teams anticipated as much time as they spent: $51 \%$ for stage 1 and $52 \%$ for stage 2 . In stage 1, $36 \%$ spent (much) more time than anticipated and $13 \%$ spent (much) less time. In stage 2, 33\% spent (much) more time than anticipated and $15 \%$ spent (much) less time. 


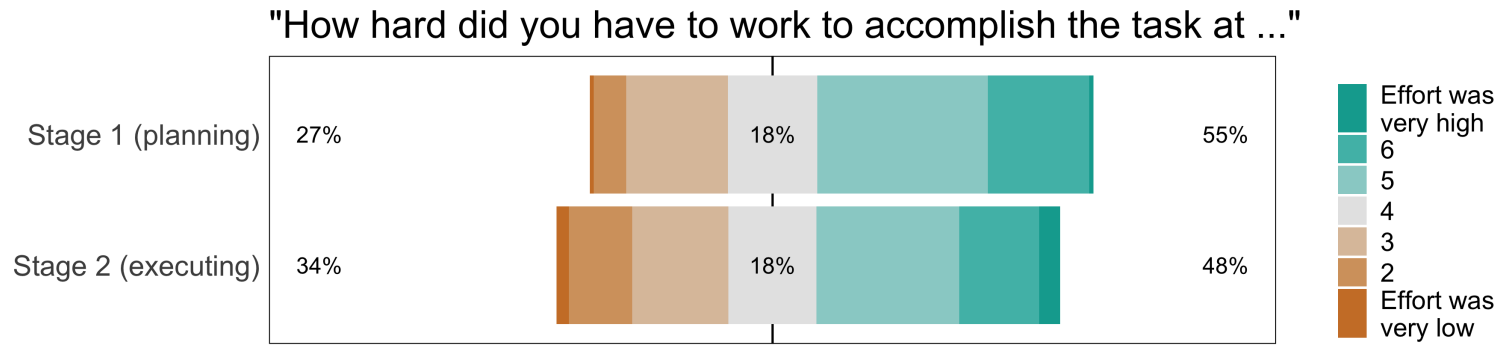

"How frustrated were you (e.g., did you feel insecure, discouraged, irritated, stressed, or annoyed) at ..."

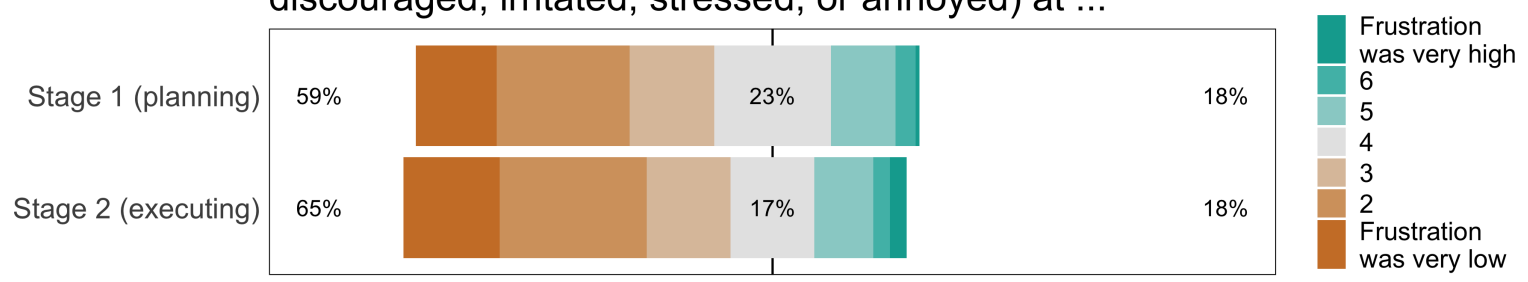

"Did you spend more time than you anticipated for ..."

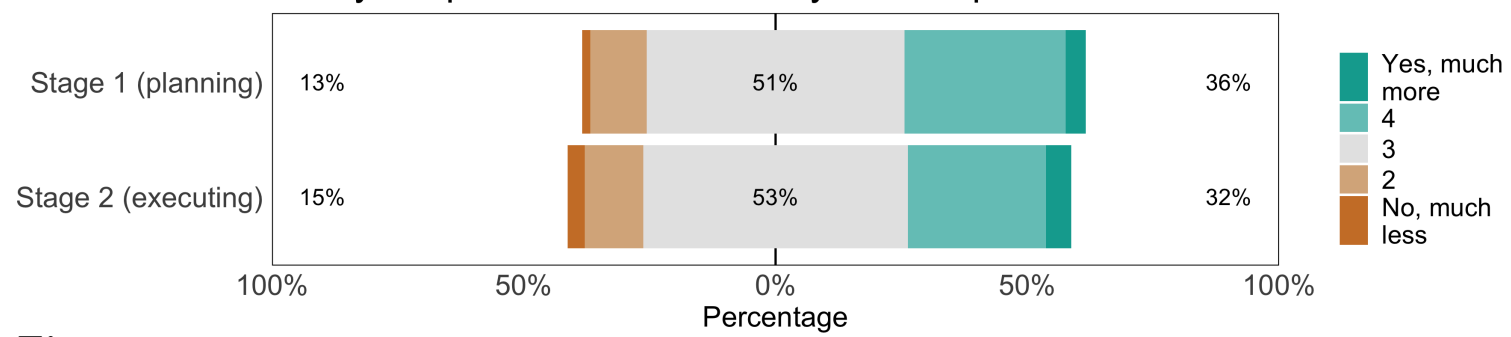

Figure 10

Responses to the survey questions about effort (top), frustration (middle), and workload (bottom). For each question, the top bar represents the teams' answers about stage 1 (planning) and the bottom bar represents the teams' answers about stage 2 (executing). For each item, the number to the left of the data bar (in brown/orange) indicates the percentage of teams that considered effort/frustration/workload (very) low. The number in the center of the data bar (in grey) indicates the percentage of teams that were neutral. The number to the right of the data bar (in green/blue) indicates the percentage of teams that considered effort/frustration/workload (very) high.

\section{Correlation between Effect Sizes and Subjective Beliefs}

Following Silberzahn et al. (2018) we explored whether the reported effect sizes were positively related to subjective beliefs about the plausibility of the research question before and after analyzing the data. This hypothesis was tested against the null-hypothesis that there is no relation between reported effect sizes and subjective beliefs. As the subjective beliefs were measured on a 7-point Likert scale, we used a rank-based Spearman correlation 
test with a Uniform[0, 1] prior (van Doorn et al., 2020).

For research question 1, we obtained strong evidence against a positive relation between prior beliefs about the plausibility of the research question and the reported effect sizes: $\mathrm{BF}_{+0}=0.03 ; \mathrm{BF}_{0+}=30.34, \rho_{s}=-0.21,95 \%$ credible interval $[-0.37,-0.04]$. In addition, we found moderate evidence against a positive relation between posterior beliefs about the plausibility of the research question and the reported effect sizes: $\mathrm{BF}_{+0}=0.31$; $\mathrm{BF}_{0+}=3.18, \rho_{s}=0.10,95 \%$ credible interval $[-0.08,0.27]$.

For research question 2, we found moderate evidence against a positive relation between prior beliefs about the plausibility of the research question and the reported effect sizes: $\mathrm{BF}_{+0}=0.12 ; \mathrm{BF}_{0+}=8.55, \rho_{s}=0.01,95 \%$ credible interval $[-0.16,0.18]$. For the posterior beliefs, however, we obtained strong evidence in favor of a positive relation between posterior beliefs about the plausibility of the research question and the reported effect sizes: $\mathrm{BF}_{+0}=67.39, \rho_{s}=0.33,95 \%$ credible interval $[0.15,0.46]$.

To further investigate changes in belief over the course of the project, we assessed the correlation between the reported effect sizes and the change in belief (i.e., the difference between posterior and prior beliefs for both research questions). For research question 1, there was basically no evidence for or against a positive relation between effect size and change in belief: $\mathrm{BF}_{+0}=1.81, \rho_{s}=0.18,95 \%$ credible interval $[0.01,0.33]$. For research question 2 on the other hand, we obtained moderate evidence that effect sizes were positively related to change in subjective belief about the plausibility of the hypothesis: $\mathrm{BF}_{+0}=9.88$, $\rho_{s}=0.24,95 \%$ credible interval $[0.07,0.39]$.

These results regarding prior beliefs provide no indication that expectations and confirmation bias influenced the teams' results. For the posterior beliefs, on the other hand, it seems that the teams updated their beliefs about the plausibility of research question 2 based on the results of their analyses. Note, however, that based on the scatterplot in Figure 11D, we should not put too much weight on this finding, as it may be partly driven by two outliers. For research question 1, the updating of beliefs may not have happened because prior beliefs about research question 1 were already in line with the outcomes, i.e., 
A. Research question 1

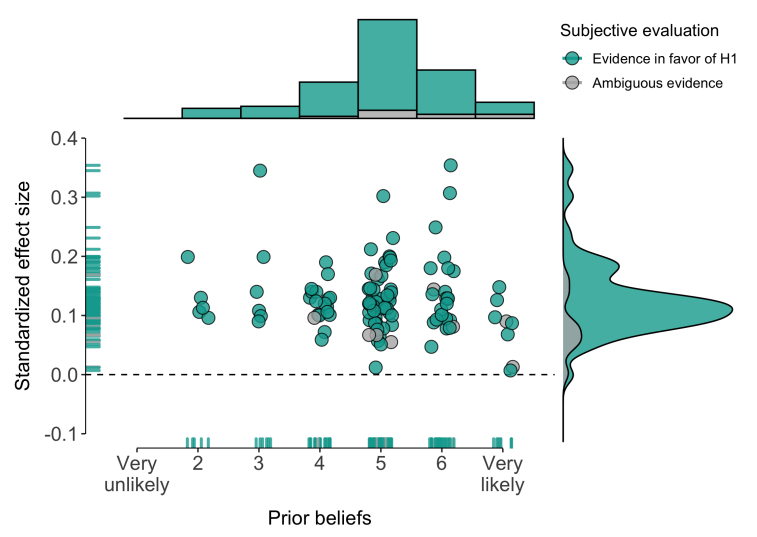

C. Research question 2

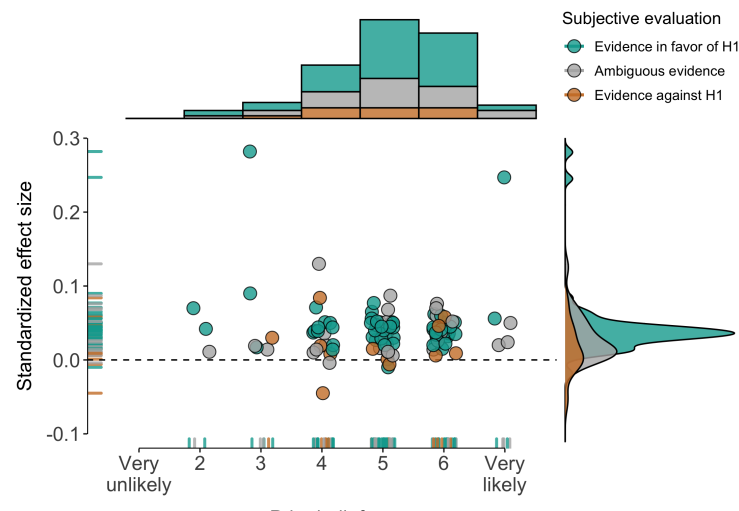

B. Research question 1

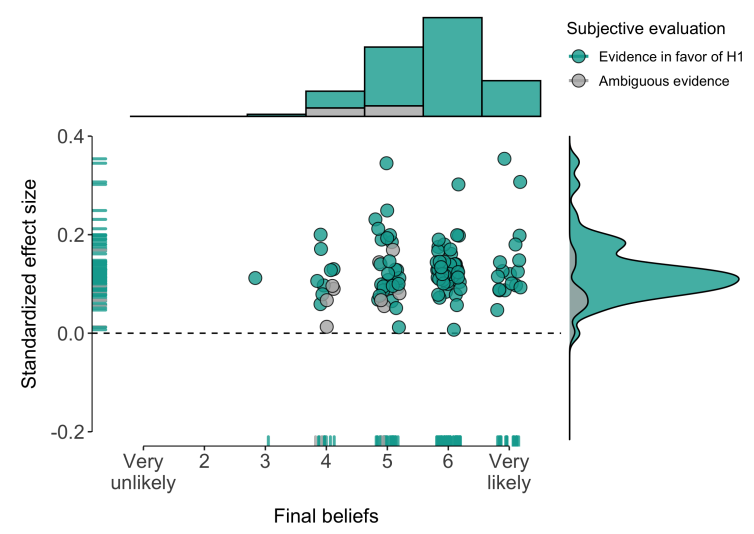

D. Research question 2

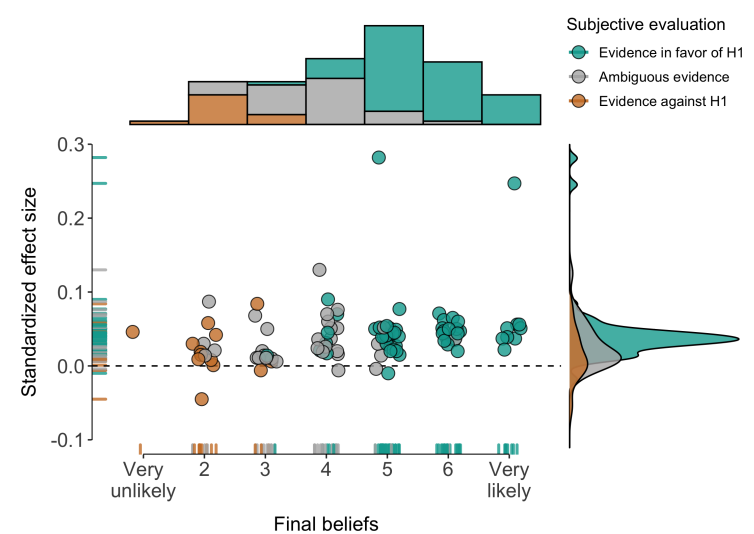

Figure 11

Reported effect sizes (beta coefficients) and subjective beliefs about the likelihood of the hypothesis. $\boldsymbol{A}$. shows the relation between effect size and prior beliefs for research question 1, B. shows the relation between effect size and final beliefs for research question 1, C. shows the relation between effect size and prior beliefs for research question 2, and D. shows the relation between effect size and final beliefs for research question 2. Points are jittered on the $x$-axis to enhance visibility. The dashed line represents an effect size of 0 . The data are separated by subjective evaluation of the evidence; green/blue points reflect the conclusion that there is good evidence for the hypothesis, grey points reflect the conclusion that the evidence is ambiguous, and brown/orange points indicate the conclusion that there is good evidence against the hypothesis. Histograms at the top represent the distribution of subjective beliefs and the density plots on the right represent the distribution of reported effect sizes.

most teams expected and reported evidence for a positive relation between religiosity and well-being, with little variation between teams.

Finally, we assessed whether reported effect sizes were related to self-reported expertise. Here, we used a Uniform[-1, 1] prior and an undirected test. This hypothesis 
was tested against the null-hypothesis that reported effect sizes and self-reported expertise were not related. For research question 1, we found moderate evidence against a correlation between effect sizes and methodological knowledge $\left(\mathrm{BF}_{10}=0.13 ; \mathrm{BF}_{01}=7.80, \rho_{s}=0.03\right.$, $95 \%$ credible interval $[-0.17,0.21])$ and weak evidence against a correlation between effect sizes and theoretical knowledge $\left(\mathrm{BF}_{10}=0.48 ; \mathrm{BF}_{01}=2.09, \rho_{s}=-0.16,95 \%\right.$ credible interval $[-0.31,0.03])$. For research question 2 , we again obtained moderate evidence against a relation between effect sizes and methodological knowledge $\left(\mathrm{BF}_{10}=0.12 ; \mathrm{BF}_{01}=8.00\right.$, $\rho_{s}=0.02,95 \%$ credible interval $\left.[-0.17,0.20]\right)$ and moderate evidence against a correlation between effect sizes and theoretical knowledge $\left(\mathrm{BF}_{10}=0.16 ; \mathrm{BF}_{01}=6.41, \rho_{s}=-0.08,95 \%\right.$ credible interval $[-0.24,0.09])$. See Figure 12 for scatterplots of the data.

\section{Summary}

In the current project, 120 analysis teams were given a large cross-cultural dataset ( $N=10,535,24$ countries) in order to investigate two research questions: (1) "Do religious people self-report higher well-being?" and (2) "Does the relation between religiosity and self-reported well-being depend on perceived cultural norms of religion?". In a two-stage procedure, the teams first proposed an analysis and then executed their planned analysis on the data.

Perhaps surprisingly in light of previous many-analysts projects, results were fairly consistent across teams. For research question 1 on the relation between religiosity and self-reported well-being, all but three teams reported a positive effect size and confidence/credible intervals that exclude zero. For research question 2, the results were somewhat more variable: $95 \%$ of the teams reported a positive effect size for the moderating influence of cultural norms of religion on the association between religiosity and self-reported well-being, with $65 \%$ of the confidence/credible intervals excluding zero. While most teams used (multilevel) linear regression, there was considerable variability in the choice of items used to construct the independent variable, the dependent variable, and the included covariates. 
A. Research question 1

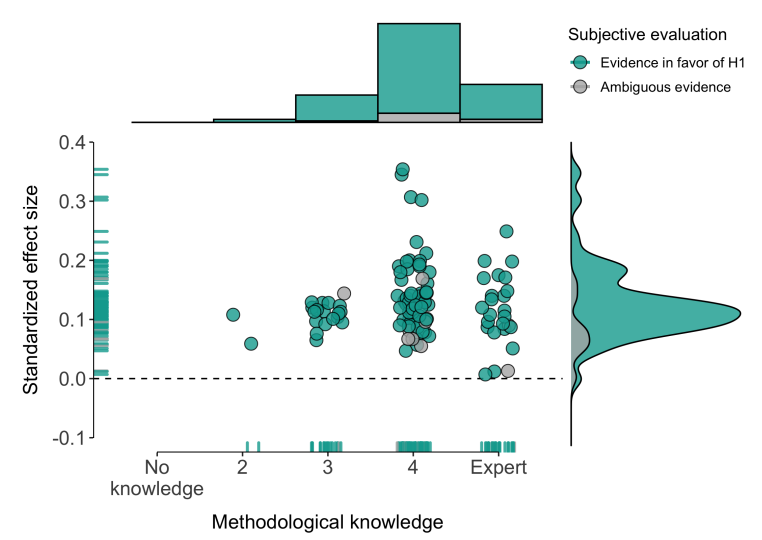

C. Research question 2

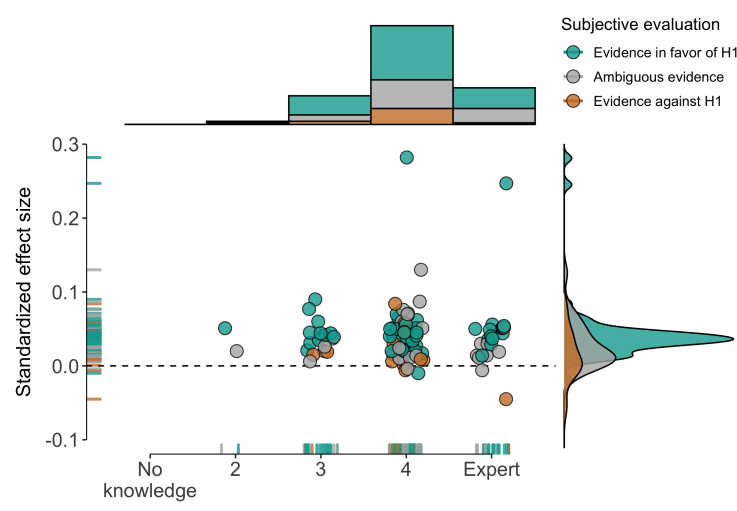

B. Research question 1

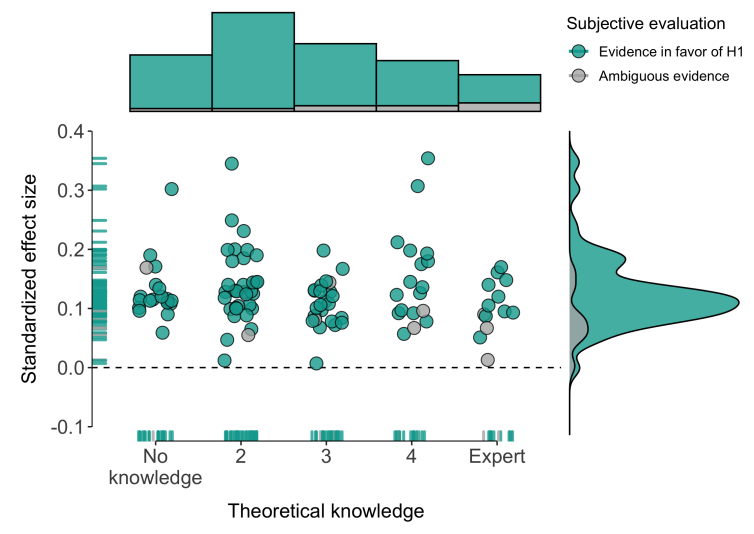

D. Research question 2

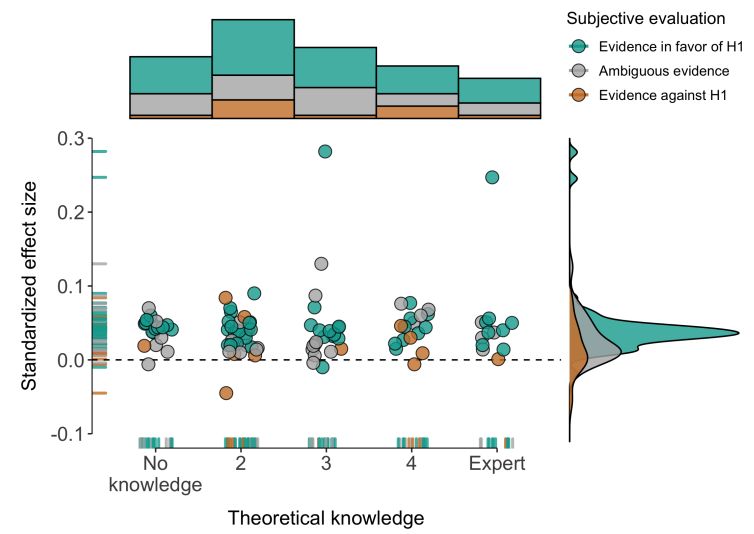

Figure 12

Reported effect sizes (beta coefficients) and self-reported team expertise. A. shows the relation between effect size for research question 1 and methodological knowledge, $\boldsymbol{B}$. shows the relation between effect size for research question 1 and theoretical knowledge, $C$. shows the relation between effect size and for research question 2 and methodological knowledge, and $\boldsymbol{D}$. shows the relation between effect size for research question 2 and theoretical knowledge. Points are jittered on the $x$-axis to enhance visibility. The dashed line represents an effect size of 0 . The data are separated by subjective evaluation of the evidence; green/blue points reflect the conclusion that there is good evidence for the hypothesis, grey points reflect the conclusion that the evidence is ambiguous, and brown/orange points indicate the conclusion that there is good evidence against the hypothesis. Histograms at the top represent the distribution of reported expertise and the density plots on the right represent the distribution of reported effect sizes.

A further discussion of these results including limitations and broader implications, as well as a reflection on the many-analysts approach is covered in the closing article (Hoogeveen et al., in preparation). There, we also address the commentaries written by some of the analysis teams. 


\section{Author Contributions}

Contributorship was documented with CRediT taxonomy using tenzing (Holcombe et al., 2020).

Conceptualization: S.H., A.S., M.v.E., and E.-J.W.

Data curation: S.H. and A.S.

Formal analysis: S.H., A.S., B.A., Y. Aditya, A.J.A., P.J.A., S. Alzahawi, Y. Amir, F.V.A., O.K.A., Q.D.A., A. Baimel, M.B.-I., M. Balsamo, S.B., F.B., M. Becerra, B.B., J. Beitner, T. Bendixen, J.B.B., M.I.B., J. Billingsley, T. Bortolini, H.B., A. Bret, F.L.B., J. Brown, C.C.B., J. Buczny, J. Bulbulia, S. Caballero, L.C., C.L.C., M.E.G.V.C., S.J.C., S. Claessens, M.C.P., A.B.C., D.L.C., S. Czoschke, C.C., E.D.D.U., Ö.D., A.D.R., H.D., J.D.R., Y.A.d.V., K.K.D., B.J.D., D.J.D., J.K.D., T.D., L.D., M.D., Y.D., T.E., P.A.E., A.E., C.T.E., S. Farahmand, H.F., M.F., A.A.F., K.F., R.F., D.F.-T., Z.F., S. Frick, L.K.F., D.G., E. Gerdin, L.G., O.G., E. Gielens, V.G., H.R.H., I.H., P.H.P.H., C.E.H., B.C.H., L.E.H., M.I., H.I., M.L.I., C.-G.I., O.I., D.I., B.J., K.A.J., J.J., J.A.K., K.K.H., E.K., B.A.K., L.A.K., S. Kelchtermans, J.M.K., R.A.K., B.K., M.L.K., M.K., D.K., J.K., S. Kritzler, A.-M.K., T.K., T.L.L., R.L., G.A.L.F., A.L., B.J.L., R.F.L., P.L., J.L., P.Ł., A.L.L., E.M., G.M.M.-R., M. Maier, D.R.M., N.M., M. Martinie, I.M., S.E.M., A.L.M., P. McAleer, T.M., M. McCullough, C.M.M., A.A.M., K.K.M., B. Mercier, P. Mitkidis, B. Monin, J.W.M., D.M., J. Morgan, J. Murphy, G.M., C.N., T.N., L.N., N.H., G.N., P.N., A.N., M.B.N., A.O.-C., L.O., Y.G.P., J.O.P., H.I.P., H.P., H.K.P., M. Pinus, S.P., V.P., M. Porubanova, M.J.P., J.M.P., M.A.P., J.P., C.P., B.G.P., J.Q.P., M.L.R., G.R., A. Roberts, L.M.R.L., R.M.R., P.R., N.R., S.-M.K.S., J.Y.S., C. Schaumans, B. Schivinski, M.C.S., S.A.S., M. Schnuerch, M.R.S., V.S., S. Sebben, S.C.S., B. Seryczyńska, U.S., M. Simsek, W.W.A.S., E.R.S., W.J.S., M. Späth, C. Spörlein, W.S., A.H.S., S. Stuber, J.S., C. Suwartono, S. Syropoulos, B. Szaszi, P.S., L.T., R.T.T., B.T., C.M.T., J.T., S.D.T., A.-M.U., R.C.M.V.A., M.A.L.M.v.A., P.V.C., O.R.V.d.A., I.V.d.C., J.V.d.N., N.N.N.v.D., C.J.V.L., V.v.M., D.v.R., C.J.J.v.Z., L.A.V., B. Većkalov, B. Verschuere, M.V., F.V., A.V., V.V., L.V.D.E.V., S. Watanabe, C.J.M.W., K.W., S. Wiechert, Z.W., M.W., C.V.O.W., 
D.W., X.Y., D.J.Y., O.Y., N.Z., Y.Z., and J.Z.

Funding acquisition: A.S., M.v.E., E.-J.W., S. Altay, N.L., R.M., and R.M.R.

Investigation: S.H., A.S., M.v.E., E.-J.W., S. Altay, T. Bendixen, R.B., K.H., R.M., L.Q.,

A. Rabelo, J.E.R., R.M.R., R.W., and D.X.

Methodology: S.H., A.S., M.v.E., and E.-J.W.

Project administration: S.H., A.S., M.v.E., and E.-J.W.

Supervision: M.v.E. and E.-J.W.

Validation: S.H. and A.S.

Visualization: S.H., A.S., and P.J.A.

Writing - original draft: S.H., A.S., M.v.E., and E.-J.W.

Writing - review \& editing: P.A.E., P.H.P.H., R.M., C.M.M., J. Murphy, T.N., J.E.R., R.M.R., S.C.S., B.T., R.C.M.V.A., M.A.L.M.v.A., and C.J.M.W.

\section{Conflicts of Interest}

The authors declare that there were no conflicts of interest with respect to the authorship or the publication of this article.

\section{Acknowledgements}

We thank Arik Cheshin, Claudio Gentili, Raluca Georgescu, Christopher Kavanagh, Alejandra Neely, Hugh Turpin, and Filip Uzarevic for their assistance in creating the material and collecting the cross-cultural data.

\section{Author List}

Suzanne Hoogeveen ${ }^{1}$, Alexandra Sarafoglou ${ }^{1}$, Balazs Aczel ${ }^{2}$, Yonathan Aditya ${ }^{3}$, Alexandra J. Alayan ${ }^{4}$, Peter J Allen ${ }^{5}$, Sacha Altay $^{6}$, Shilaan Alzahawi ${ }^{7}$, Yulmaida Amir ${ }^{8}$, Francis-Vincent Anthony ${ }^{9}$, Obed Kwame Appiah ${ }^{1}$, Quentin D. Atkinson ${ }^{10}$, Adam Baimel ${ }^{11}$, Merve Balkaya-Ince ${ }^{12}$, Michela Balsamo ${ }^{13}$, Sachin Banker ${ }^{14}$, František Bartoš ${ }^{15}$, Mario Becerra $^{16}$, Bertrand Beffara ${ }^{17}$, Julia Beitner ${ }^{18}$, Theiss Bendixen ${ }^{19}$, Jana B. Berkessel ${ }^{20}$, 
Renatas Berniūnas ${ }^{21}$, Matthew I. Billet ${ }^{22}$, Joseph Billingsley ${ }^{23}$, Tiago Bortolini2 ${ }^{24}$, Heiko Breitsohl $^{25}$, Amélie Bret ${ }^{26}$, Faith L Brown ${ }^{27}$, Jennifer Brown ${ }^{28}$, Claudia C. Brumbaugh ${ }^{29}$, Jacek Buczny ${ }^{30}$, Joseph Bulbulia ${ }^{31}$, Saúl Caballero ${ }^{32}$, Leonardo Carlucci ${ }^{33}$, Cheryl L. Carmichael $^{34}$, Marco E. G. V. Cattaneo ${ }^{35}$, Sarah J. Charles ${ }^{28}$, Scott Claessens ${ }^{10}$, Maxinne C. Panagopoulos ${ }^{36}$, Angelo Brandelli Costa ${ }^{37}$, Damien L. Crone ${ }^{38}$, Stefan Czoschke ${ }^{18}$, Christian Czymara ${ }^{39}$, E. Damiano D’Urso ${ }^{40}$, Örjan Dahlström ${ }^{41,42}$, Anna Dalla Rosa ${ }^{43}$, Henrik Danielsson ${ }^{41,42}$, Jill De Ron ${ }^{1}$, Ymkje Anna de Vries ${ }^{44,45}$, Kristy K. Dean ${ }^{46}$, Bryan J. Dik ${ }^{4}$, David J. Disabato ${ }^{47}$, Jaclyn K. Doherty ${ }^{48}$, Tim Draws ${ }^{49}$, Lucas Drouhot ${ }^{50}$, Marin Dujmovic ${ }^{5}$, Yarrow Dunham ${ }^{51}$, Tobias Ebert ${ }^{20}$, Peter A. Edelsbrunner ${ }^{52}$, Anita Eerland $^{53}$, Christian T. Elbaek ${ }^{54}$, Shole Farahmand ${ }^{55}$, Hooman Farahmand ${ }^{56}$, Miguel Farias $^{28}$, Abrey A. Feliccia ${ }^{57}$, Kyle Fischer ${ }^{10}$, Ronald Fischer ${ }^{58}$, Donna Fisher-Thompson ${ }^{59}$, Zoë Francis ${ }^{60}$, Susanne Frick ${ }^{61}$, Lisa K. Frisch ${ }^{62}$, Diogo Geraldes ${ }^{63}$, Emily Gerdin ${ }^{51}$, Linda Geven $^{64,1}$, Omid Ghasemi ${ }^{65}$, Erwin Gielens ${ }^{66}$, Vukašin Gligorić ${ }^{67}$, Kristin Hagel ${ }^{68}$, Hannah R. Hamilton ${ }^{69}$, Imaduddin Hamzah ${ }^{70}$, Paul H. P. Hanel ${ }^{71}$, Christopher E. Hawk ${ }^{72}$, Benjamin C. Holding ${ }^{73,74}$, Lina E. Homman ${ }^{41,42}$, Moritz Ingendahl ${ }^{75}$, Hilla Inkilä ${ }^{76}$, Mary L. Inman $^{77}$, Chris-Gabriel Islam ${ }^{78,79}$, Ozan Isler ${ }^{80,81}$, David Izydorczyk ${ }^{61}$, Bastian Jaeger ${ }^{40}$, Kathryn A. Johnson ${ }^{82}$, Jonathan Jong ${ }^{83}$, Johannes A. Karl ${ }^{31}$, Karel K. Himawan ${ }^{84}$, Erikson Kaszubowski ${ }^{85}$, Benjamin A. Katz ${ }^{86}$, Lucas A Keefer ${ }^{27}$, Stijn Kelchtermans ${ }^{16}$, John M. Kelly ${ }^{87}$, Richard A. Klein ${ }^{40}$, Bennett Kleinberg ${ }^{88}$, Megan L. Knowles ${ }^{89}$, Marta Kołczyńska $^{90}$, Dave Koller ${ }^{91}$, Julia Krasko ${ }^{92}$, Sarah Kritzler ${ }^{92}$, Angelos-Miltiadi Krypotos ${ }^{93}$, Thanos Kyritsis ${ }^{10}$, Todd (Larson) Landes ${ }^{94}$, Ruben Laukenmann ${ }^{95}$, Guy A. Lavender Forsyth $^{10}$, Aryeh Lazar ${ }^{96}$, Barbara J. Lehman ${ }^{97}$, Neil Levy ${ }^{98}$, Ronda F. Lo ${ }^{99}$, Paul Lodder $^{100}$, Jennifer Lorenz ${ }^{78}$, Paweł Łowicki ${ }^{101}$, Albert L. Ly ${ }^{102}$, Esther Maassen ${ }^{40}$, Gina M Magyar-Russell ${ }^{103}$, Maximilian Maier ${ }^{1}$, Dylan R. Marsh ${ }^{4}$, Nuria Martinez ${ }^{104}$, Marcellin Martinie $^{94}$, Ihan Martoyo ${ }^{3}$, Susan E. Mason ${ }^{59}$, Anne Lundahl Mauritsen ${ }^{19}$, Phil McAleer ${ }^{36}$, Thomas McCauley $^{105}$, Michael McCullough ${ }^{105}$, Ryan McKay ${ }^{106}$, Camilla M. McMahon ${ }^{107}$, Amelia A. McNamara ${ }^{108}$, Kira K. Means ${ }^{97}$, Brett Mercier ${ }^{109}$, Panagiotis Mitkidis ${ }^{110}$, Benoît Monin $^{7}$, Jordan W. Moon ${ }^{82}$, David Moreau ${ }^{111}$, Jonathan Morgan ${ }^{112,113}$, James Murphy ${ }^{104}$, 
George Muscatt ${ }^{114}$, Christof Nägel ${ }^{115}$, Tamás Nagy ${ }^{116}$, Ladislas Nalborczyk ${ }^{117,118}$, Nandor Hajdu ${ }^{119,120}$, Gustav Nilsonne ${ }^{121}$, Pamina Noack ${ }^{122}$, Ara Norenzayan ${ }^{22}$, Michèle B. Nuijten $^{40}$, Anton Olsson-Collentine ${ }^{40}$, Lluis Oviedo ${ }^{123}$, Yuri G. Pavlov ${ }^{124,125}$, James O. Pawelski $^{38}$, Hannah I. Pearson ${ }^{126}$, Hugo Pedder ${ }^{127}$, Hannah K. Peetz ${ }^{128}$, Michael Pinus ${ }^{129}$, Steven Pirutinsky ${ }^{130}$, Vince Polito ${ }^{131}$, Michaela Porubanova ${ }^{132}$, Michael J. Poulin ${ }^{133}$, Jason M Prenoveau ${ }^{103}$, Mark A. Prince ${ }^{4}$, John Protzko ${ }^{134}$, Campbell Pryor ${ }^{94}$, Benjamin G. Purzycki $^{19}$, Lin Qiu ${ }^{135}$, Julian Quevedo Pütter ${ }^{61}$, André Rabelo ${ }^{136}$, Milen L. Radell ${ }^{59}$, Jonathan E. Ramsay ${ }^{137}$, Graham Reid ${ }^{138,139}$, Andrew Roberts ${ }^{65}$, Lindsey M. Root Luna ${ }^{77}$, Robert M. Ross ${ }^{140}$, Piotr Roszak ${ }^{141}$, Nirmal Roy ${ }^{49}$, Suvi-Maria K. Saarelainen ${ }^{76}$, Joni Y. Sasaki ${ }^{126}$, Catherine Schaumans ${ }^{142}$, Bruno Schivinski ${ }^{143}$, Marcel C. Schmitt ${ }^{144}$, Sarah A. Schnitker ${ }^{12}$, Martin Schnuerch ${ }^{95}$, Marcel R. Schreiner ${ }^{61}$, Victoria Schüttengruber ${ }^{62}$, Simone Sebben ${ }^{145}$, Suzanne C. Segerstrom ${ }^{146}$, Berenika Seryczyńska ${ }^{147}$, Uffe Shjoedt ${ }^{19}$, Müge Simsek ${ }^{148}$, Willem W. A. Sleegers ${ }^{40}$, Eliot R. Smith ${ }^{149}$, Walter J. Sowden ${ }^{150}$, Marion Späth ${ }^{122}$, Christoph Spörlein ${ }^{151}$, William Stedden ${ }^{152}$, Andrea H. Stoevenbelt ${ }^{40,153}$, Simon Stuber ${ }^{154}$, Justin Sulik ${ }^{155}$, Christiany Suwartono ${ }^{156}$, Stylianos Syropoulos ${ }^{157}$, Barnabas Szaszi $^{120}$, Peter Szecsi ${ }^{119,120}$, Louis Tay ${ }^{158}$, Robert T. Thibault ${ }^{159,5}$, Burt Thompson ${ }^{59}$, Christian M. Thurn ${ }^{52}$, Josefa Torralba ${ }^{160}$, Shelby D. Tuthill ${ }^{4}$, Ann-Marie Ullein ${ }^{122}$, Robbie C. M. Van Aert ${ }^{40}$, Marcel A.L.M. van Assen ${ }^{40}$, Patty Van Cappellen ${ }^{161}$, Olmo R. Van den Akker ${ }^{40}$, Ine Van der Cruyssen ${ }^{1,162}$, Jolanda Van der $\mathrm{Noll}^{163}$, Noah N N van Dongen $^{164}$, Caspar J Van Lissa ${ }^{165}$, Valerie van Mulukom²8, Don van Ravenzwaaij ${ }^{166}$, Casper J J van Zyl ${ }^{167}$, Leigh Ann Vaughn ${ }^{57}$, Bojana Većkalov ${ }^{67}$, Bruno Verschuere ${ }^{1}$, Michelangelo Vianello ${ }^{43}$, Felipe Vilanova ${ }^{37}$, Allon Vishkin ${ }^{168}$, Vera Vogel ${ }^{75}$, Leonie V.D.E. Vogelsmeier $^{40}$, Shoko Watanabe ${ }^{169}$, Cindel J. M. White ${ }^{99}$, Kristina Wiebels ${ }^{111}$, Sera Wiechert $^{1,162}$, Zachary Willett ${ }^{97}$, Maciej Witkowiak ${ }^{170}$, Charlotte V. O. Witvliet ${ }^{77}$, Dylan Wiwad ${ }^{171}$, Robin Wuyts ${ }^{1}$, Dimitris Xygalatas ${ }^{172}$, Xin Yang ${ }^{51}$, Darren J. Yeo ${ }^{173,174}$, Onurcan Yilmaz ${ }^{175}$, Natalia Zarzeczna ${ }^{1}$, Yitong Zhao ${ }^{176}$, Josjan Zijlmans ${ }^{177}$, Michiel van Elk $^{64}$, Eric-Jan Wagenmakers ${ }^{1}$ 
${ }^{1}$ University of Amsterdam, ${ }^{2}$ Institute of Psychology, ELTE University, Budapest, Hungary, ${ }^{3} \mathrm{UPH},{ }^{4}$ Colorado State University, ${ }^{5}$ School of Psychological Science, University of Bristol, ${ }^{6}$ Institut Jean Nicod, Paris, France, ${ }^{7}$ Stanford University, Graduate School of Business, ${ }^{8}$ Universitas Muhammadiyah Prof. Dr. HAMKA, ${ }^{9}$ Salesian University, Rome, ${ }^{10}$ University of Auckland, ${ }^{11}$ Centre for Psychological Research, Oxford Brookes University, ${ }^{12}$ Department of Psychology and Neuroscience, Baylor University, ${ }^{13}$ Department of Psychological, Health and Territorial Sciences, University of Chieti, Chieti, Italy, ${ }^{14}$ University of Utah, Eccles School of Business, ${ }^{15}$ Department of Psychological Methods, University of Amsterdam, ${ }^{16} \mathrm{KU}$ Leuven, ${ }^{17}$ The Walden III Slowpen Science Laboratory, ${ }^{18}$ Institute of Psychology, Goethe University Frankfurt, Germany, ${ }^{19}$ Department of the Study of Religion, Aarhus University, ${ }^{20}$ Mannheim Centre for European Social Research, University of Mannheim, ${ }^{21}$ Vilnius University, Lithuania, ${ }^{22}$ University of British Columbia, ${ }^{23}$ North Carolina State University, ${ }^{24}$ D'Or Institute for Research and Education (IDOR), ${ }^{25}$ University of Klagenfurt, Austria, ${ }^{26}$ University of Nantes, ${ }^{27}$ The University of Southern Mississippi, ${ }^{28}$ Coventry University, ${ }^{29}$ Queens College, City University of New York, ${ }^{30}$ Department of Experimental and Applied Psychology, VU Amsterdam, ${ }^{31}$ Victoria University of Wellington, ${ }^{32}$ Instituto Tecnológico Autónomo de México, ${ }^{33}$ Department of Economics, Management and Territory, University of Foggia, Italy, ${ }^{34}$ Brooklyn College \& Graduate Center, City University of New York, ${ }^{35}$ Department of Clinical Research, University of Basel, Switzerland, ${ }^{36}$ University of Glasgow, ${ }^{37}$ Pontifícia Universidade Católica do Rio Grande do Sul, ${ }^{38}$ University of Pennsylvania, ${ }^{39}$ Department of Sociology with Focus on Quantitative Methods for Social Research, Goethe University Frankfurt, ${ }^{40}$ Tilburg University, ${ }^{41}$ Linköping University, ${ }^{42}$ The Swedish Institute for Disability Research, ${ }^{43}$ Department of Philosophy, Sociology, Education and Applied Psychology, University of Padua, ${ }^{44}$ Department of Developmental Psychology, University of Groningen, Groningen, NL, ${ }^{45}$ Interdisciplinary Center Psychopathology and Emotion Regulation, University Medical Center Groningen, Groningen, NL, ${ }^{46}$ Grand Valley State University, ${ }^{47}$ Kent State University, ${ }^{48}$ The Graduate Center, City University of New York, ${ }^{49}$ Delft University of Technology, ${ }^{50}$ Utrecht 
University, ${ }^{51}$ Yale University, ${ }^{52}$ ETH Zurich, ${ }^{53}$ Department of Communication Science, Radboud University, ${ }^{54}$ Aarhus University, ${ }^{55} \mathrm{PhD}$ student, Faculty of Physical Education and Sport Science, University of Tehran, ${ }^{56}$ Master graduated, Clinical Psychology, Faculty of Education and Psychology, Shiraz University, ${ }^{57}$ Ithaca College, Ithaca, NY, USA, ${ }^{58}$ Victoria University of Wellington \& IDOR, ${ }^{59}$ Niagara University, ${ }^{60}$ University of the Fraser Valley, ${ }^{61}$ Department of Psychology, School of Social Sciences, University of Mannheim, ${ }^{62}$ University of Zürich, ${ }^{63}$ Utrecht School of Economics, Utrecht University, ${ }^{64}$ Leiden University, ${ }^{65}$ School of Psychological Sciences, Macquarie University, ${ }^{66}$ Tilburg Universty, ${ }^{67}$ Department of Psychology, University of Amsterdam, ${ }^{68}$ Max Planck Institute for Evolutionary Anthropology, Germany, ${ }^{69}$ UConn School of Medicine, ${ }^{70}$ Politeknik Ilmu Pemasyarakatan, ${ }^{71}$ University of Essex, ${ }^{72}$ DigiPen Institute of Technology, Department of Humanities and Social Sciences, ${ }^{73}$ University of Copenhagen, Faculty of Social Science, Department of Sociology, ${ }^{74}$ Karolinska Institutet, Department of Clinical Neuroscience., ${ }^{75}$ Universität Mannheim, ${ }^{76}$ University of Eastern Finland, ${ }^{77}$ Hope College, ${ }^{78}$ University of Göttingen, ${ }^{79}$ Federal Statistical Office of Germany, ${ }^{80}$ School of Economics and Finance, Queensland University of Technology, Brisbane, Australia, ${ }^{81}$ Centre for Behavioural Economics, Society and Technology, Brisbane, Australia., ${ }^{82}$ Arizona State University, Department of Psychology, ${ }^{83}$ Centre for Trust, Peace and Social Relations, Coventry University, ${ }^{84}$ Faculty of Psychology, Universitas Pelita Harapan, ${ }^{85}$ Universidade Federal de Santa Catarina, ${ }^{86}$ SUNY Stony Brook University, ${ }^{87}$ Department of Psychological Science, University of California, Irvine, ${ }^{88}$ Tilburg University \& University College London, ${ }^{89}$ Franklin \& Marshall College, ${ }^{90}$ Institute of Political Science of the Polish Academy of Sciences, ${ }^{91}$ University of Zurich, University of Amsterdam, ${ }^{92}$ Faculty of Psychology, Ruhr University Bochum, ${ }^{93}$ Utrecht University \& KU Leuven, ${ }^{94}$ University of Melbourne, ${ }^{95}$ University of Mannheim, ${ }^{96}$ Ariel University, Israel, ${ }^{97}$ Western Washington University, ${ }^{98}$ Department of Philosophy, Macquarie University, Australia, ${ }^{99}$ York University, ${ }^{100}$ Department of Methodology \& Statistics, Tilburg University, ${ }^{101}$ Faculty of Psychology, Univeristy of Warsaw, Poland, ${ }^{102}$ Department of Psychology, Loma Linda University, ${ }^{103}$ Loyola University Mary- 
land, ${ }^{104}$ Canterbury Christ Church University, ${ }^{105}$ University of California, San Diego, ${ }^{106}$ Royal Holloway, University of London, Egham, UK, ${ }^{107}$ Miami University, ${ }^{108}$ University of St Thomas, ${ }^{109}$ Department of Psychology, University of Toronto, ${ }^{110}$ Aarhus University \& Duke University, ${ }^{111}$ School of Psychology \& Centre for Brain Research, The University of Auckland, ${ }^{112}$ University of Colorado at Colorado Springs, ${ }^{113}$ Center for Mind and Culture, ${ }^{114}$ School of Life Sciences, University of Warwick, ${ }^{115}$ German Police University, ${ }^{116}$ Institute of Psychology, ELTE Eotvos Lorand University, Budapest, Hungary, ${ }^{117}$ Aix Marseille Univ, CNRS, LPC, Marseille, France, ${ }^{118}$ Aix Marseille Univ, CNRS, LNC, Marseille, France, ${ }^{119}$ Doctoral School of Psychology, ELTE Eötvös Loránd University, Budapest, Hungary, ${ }^{120}$ Institute of Psychology, ELTE Eötvös Loránd University, Budapest, Hungary, ${ }^{121}$ Karolinska Institutet, Department of Clinical Neuroscience \& Stockholm University, Department of Psychology, ${ }^{122}$ Heinrich-Heine-University Duesseldorf, ${ }^{123}$ Antonianum University, Rome, ${ }^{124}$ Ural Federal University, Ekaterinburg, Russia, ${ }^{125}$ University of Tübingen, Germany, ${ }^{126}$ University of Hawai'i at Manoa, ${ }^{127}$ Department Population Health Sciences, Bristol Medical School, University of Bristol, ${ }^{128}$ Behavioural Science Institute, Radboud University Nijmegen, ${ }^{129}$ The Hebrew University of Jerusalem, ${ }^{130}$ Touro College, Graduate School of Social Work, ${ }^{131}$ School of Psychological Sciences, Macquarie University, Australia, ${ }^{132}$ Microsoft Mixed Reality, ${ }^{133}$ University at Buffalo, ${ }^{134}$ Central Connecticut State University, ${ }^{135}$ Nanyang Technological University, Singapore, ${ }^{136}$ Universidade de Brasília, Brazil, ${ }^{137}$ James Cook University, Singapore, ${ }^{138}$ Department of Experimental Psychology, Medical Sciences Division, University of Oxford, ${ }^{139}$ Heart \& Brain Group, Wellcome Centre for Integrative Neuroimaging (WIN), Department of Psychiatry, Medical Sciences Division, University of Oxford, ${ }^{140}$ Department of Psychology, Macquarie University, Australia, ${ }^{141}$ Copernicus University of Torun, ${ }^{142}$ independent researcher, ${ }^{143}$ School of Media and Communication, RMIT University, ${ }^{144}$ Department of Psychology, University of KoblenzLandau, ${ }^{145}$ University of Zurich, ${ }^{146}$ Department of Psychology, University of Kentucky, ${ }^{147}$ Copernicus Univerversity of Torun, ${ }^{148}$ Netherlands Interdisciplinary Demographic Institute-KNAW, University of Groningen, ${ }^{149}$ Indiana University, Bloomington, ${ }^{150}$ Department 
of Behavioral Health, Tripler Army Medical Center, Honolulu, Hawaii, USA, ${ }^{151}$ Institut für Sozialwissenschaften, Heinrich-Heine Universität Düsseldorf, ${ }^{152}$ none, ${ }^{153}$ University of Groningen, ${ }^{154}$ Universität Koblenz Landau, ${ }^{155}$ Cognition, Values and Behaviour Lab, Ludwig Maximilian University, Germany, ${ }^{156}$ Faculty of Psychology, Atma Jaya Catholic University of Indonesia, Indonesia, ${ }^{157}$ University of Massachusetts Amherst, Department of Psychological and Brain Science, ${ }^{158}$ Purdue University, ${ }^{159}$ Meta-research Innovation Center at Stanford (METRICS), ${ }^{160}$ University of Murcia, ${ }^{161}$ Duke University, ${ }^{162}$ Hebrew University of Jerusalem, ${ }^{163}$ FernUniversität in Hagen, ${ }^{164}$ University of Amsterdam Department of Psychology, ${ }^{165}$ Utrecht University, department of Methodology \& Statistics, ${ }^{166}$ University of Groningen, Groningen, The Netherlands, ${ }^{167}$ University of Johannesburg, ${ }^{168}$ University of Michigan, ${ }^{169}$ University of Illinois at Urbana-Champaign, ${ }^{170}$ no affiliation, ${ }^{171}$ Kellogg School of Management, Northwestern University, ${ }^{172}$ University of Connecticut, USA, ${ }^{173}$ Division of Psychology, School of Social Sciences, Nanyang Technological University, Singapore, ${ }^{174}$ Department of Psychology \& Human Development, Vanderbilt University, Nashville, TN, USA, ${ }^{175}$ Department of Psychology, Kadir Has University, Istanbul, Turkey, ${ }^{176}$ Department of Psychology, Wake Forest University, ${ }^{177}$ Amsterdam University Medical Center Department of Child and Adolescent Psychiatry

\section{Funding}

Robert M. Ross was supported by Australian Research Council (grant number DP180102384); Ryan McKay was supported by Cogito Foundation [grant number R10917]; Sacha Altay was supported by French Agence Nationale de la Recherche (reference 17EURE-0017 FrontCog and 10-IDEX-0001-02 PSL); Emily Gerdin was supported by National Science Foundation Graduate Research Fellowship (GR100544); Eric-Jan Wagenmakers was supported by Netherlands Organisation for Scientific Research [grant number 016.Vici.170.083]; Don van Ravenzwaaij was supported by Netherlands Organisation for Scientific Research [grant number 016.Vidi.188.001]; Alexandra Sarafoglou was supported by Netherlands Organisation for Scientific Research [grant number 406-17-568]; Michiel 
van Elk was supported by Templeton Foundation [grant number 60663]; Damien L. Crone, James O. Pawelski, and Louis Tay were supported by Templeton Religion Trust.

\section{References}

Abdel-Khalek, A. M. (2006). Measuring happiness with a single-item scale. Social Behavior and Personality: An International Journal, 34, 139-150. https://doi.org/10.2224/ sbp.2006.34.2.139

Abu-Raiya, H. (2013). On the links between religion, mental health and inter-religious conflict: A brief summary of empirical research. The Israel Journal of Psychiatry and Related Sciences, 50(2), 130-139.

Aczel, B., Szaszi, B., Nilsonne, G., van den Akker, O. R., Albers, C. J., van Assen, M. A., Bastiaansen, J. A., Benjamin, D., Boehm, U., Botvinik-Nezer, R., Bringmann, L. F., Busch, N. A., Caruyer, E., Cataldo, A. M., Cowan, N., Delios, A., van Dongen, N. N., Donkin, C., van Doorn, J. B., ... Wagenmakers, E.-J. (2021). Consensusbased guidance for conducting and reporting multi-analyst studies (P. Rodgers, Ed.). eLife, 10, e72185. https://doi.org/10.7554/eLife.72185

Bains, S. (2011). Questioning the integrity of the John Templeton Foundation. Evolutionary Psychology, 9(1), 92-115. https://doi.org/10.1177/147470491100900111

Bastiaansen, J. A., Kunkels, Y. K., Blaauw, F. J., Boker, S. M., Ceulemans, E., Chen, M., Chow, S.-M., de Jonge, P., Emerencia, A. C., Epskamp, S., Fisher, A. J., Hamaker, E. L., Kuppens, P., Lutz, W., Meyer, M. J., Moulder, R., Oravecz, Z., Riese, H., Rubel, J., ... Bringmann, L. F. (2020). Time to get personal? The impact of researchers choices on the selection of treatment targets using the experience sampling methodology. Journal of Psychosomatic Research, 137, 110211. https://doi.org/10. 1016/j.jpsychores.2020.110211

Bergin, A. E. (1983). Religiosity and mental health: A critical reevaluation and metaanalysis. Professional Psychology: Research and Practice, 14(2), 170-184. https: //doi.org/10.1037/0735-7028.14.2.170 
Boehm, U., Annis, J., Frank, M. J., Hawkins, G. E., Heathcote, A., Kellen, D., Krypotos, A.-M., Lerche, V., Logan, G. D., Palmeri, T. J., van Ravenzwaaij, D., Servant, M., Singmann, H., Starns, J. J., Voss, A., Wiecki, T. V., Matzke, D., \& Wagenmakers, E.-J. (2018). Estimating across-trial variability parameters of the Diffusion Decision Model: Expert advice and recommendations. Journal of Mathematical Psychology, 87, 46-75. https://doi.org/10.1016/j.jmp.2018.09.004

Botvinik-Nezer, R., Holzmeister, F., Camerer, C. F., Dreber, A., Huber, J., Johannesson, M., Kirchler, M., Iwanir, R., Mumford, J. A., Adcock, R. A., Avesani, P., Baczkowski, B. M., Bajracharya, A., Bakst, L., Ball, S., Barilari, M., Bault, N., Beaton, D., Beitner, J., ... Schonberg, T. (2020). Variability in the analysis of a single neuroimaging dataset by many teams. Nature, 582(7810), 84-88. https://doi.org/10.1038/s41586020-2314-9

Captari, L. E., Hook, J. N., Hoyt, W., Davis, D. E., McElroy-Heltzel, S. E., \& Worthington Jr., E. L. (2018). Integrating clients' religion and spirituality within psychotherapy: A comprehensive meta-analysis. Journal of Clinical Psychology, 74(11), 1938-1951. https://doi.org/10.1002/jclp.22681

Chang, M.-C., Chen, P.-F., Lee, T.-H., Lin, C.-C., Chiang, K.-T., Tsai, M.-F., Kuo, H.-F., \& Lung, F.-W. (2021). The effect of religion on psychological resilience in healthcare workers during the coronavirus disease 2019 pandemic. Frontiers in Psychology, 12, 628894. https://doi.org/10.3389/fpsyg.2021.628894

Chiu, C.-Y., Gelfand, M. J., Yamagishi, T., Shteynberg, G., \& Wan, C. (2010). Intersubjective culture: The role of intersubjective perceptions in cross-cultural research. Perspectives on Psychological Science, 5(4), 482-493. https://doi.org/10.1177/ 1745691610375562

de Vrieze, J. (2018). The metawars. Science, 361(6408), 1184-1188. https://doi.org/10. 1126/science.361.6408.1184 
Diener, E., Tay, L., \& Myers, D. G. (2011). The religion paradox: If religion makes people happy, why are so many dropping out? Journal of Personality and Social Psychology, 101(6), 1278-1290. https://doi.org/10.1037/a0024402

Dressler, W. W., Balieiro, M. C., Ribeiro, R. P., \& Santos, J. E. D. (2007). Cultural consonance and psychological distress: Examining the associations in multiple cultural domains. Culture, Medicine and Psychiatry, 31(2), 195-224. https://doi.org/10. 1007/s11013-007-9046-2

Dutilh, G., Annis, J., Brown, S. D., Cassey, P., Evans, N. J., Grasman, R. P. P. P., Hawkins, G. E., Heathcote, A., Holmes, W. R., Krypotos, A.-M., Kupitz, C. N., Leite, F. P., Lerche, V., Lin, Y.-S., Logan, G. D., Palmeri, T. J., Starns, J. J., Trueblood, J. S., van Maanen, L., ... Donkin, C. (2019). The quality of response time data inference: A blinded, collaborative assessment of the validity of cognitive models. Psychonomic Bulletin ES Review, 26(4), 1051-1069. https://doi.org/10.3758/s13423-017-1417-2

Ebert, T., Gebauer, J. E., Talman, J. R., \& Rentfrow, P. J. (2020). Religious people only live longer in religious cultural contexts: A gravestone analysis. Journal of Personality and Social Psychology, 119(1), 1-6. https://doi.org/10.1037/pspa0000187

Fillard, P., Descoteaux, M., Goh, A., Gouttard, S., Jeurissen, B., Malcolm, J., RamirezManzanares, A., Reisert, M., Sakaie, K., Tensaouti, F., Yo, T., Mangin, J.-F., \& Poupon, C. (2011). Quantitative evaluation of 10 tractography algorithms on a realistic diffusion MR phantom. NeuroImage, 56(1), 220-234. https://doi.org/10.1016/ j.neuroimage.2011.01.032

Garssen, B., Visser, A., \& Pool, G. (2020). Does spirituality or religion positively affect mental health? Meta-analysis of longitudinal studies. The International Journal for the Psychology of Religion, 31(1), 4-20. https://doi.org/10.1080/10508619.2020. 1729570

Gebauer, J. E., Sedikides, C., Schönbrodt, F. D., Bleidorn, W., Rentfrow, P. J., Potter, J., \& Gosling, S. D. (2017). The religiosity as social value hypothesis: A multimethod replication and extension across 65 countries and three levels of spatial 
aggregation. Journal of Personality and Social Psychology, 113(3), e18-e39. https: //doi.org/10.1037/pspp0000104

George, L. K., Ellison, C. G., \& Larson, D. B. (2002). Explaining the relationships between religious involvement and health. Psychological Inquiry, 13(3), 190-200. https:// doi.org/10.1207/S15327965PLI1303_04

Grosz, M. P., Rohrer, J. M., \& Thoemmes, F. (2020). The taboo against explicit causal inference in nonexperimental psychology. Perspectives on Psychological Science, 15(5), 1243-1255. https://doi.org/10.1177/1745691620921521

Hackney, C. H., \& Sanders, G. S. (2003). Religiosity and mental health: A meta-analysis of recent studies. Journal for the Scientific Study of Religion, 42(1), 43-55. https: //doi.org/10.1111/1468-5906.t01-1-00160

Harris, J. I., Usset, T., Voecks, C., Thuras, P., Currier, J., \& Erbes, C. (2018). Spiritually integrated care for PTSD: A randomized controlled trial of "Building Spiritual Strength”. Psychiatry Research, 267, 420-428. https://doi.org/10.1016/j.psychres. 2018.06.045

Hayward, R. D., \& Elliott, M. (2014). Cross-national analysis of the influence of cultural norms and government restrictions on the relationship between religion and wellbeing. Review of Religious Research, 56(1), 23-43. https://doi.org/10.1007/s13644013-0135-0

Holcombe, A. O., Kovacs, M., Aust, F., \& Aczel, B. (2020). Documenting contributions to scholarly articles using CRediT and tenzing. PLoS One, 15, e0244611.

Hoogeveen, S., Haaf, J. M., Bulbulia, J. A., Ross, R. M., McKay, R., Altay, S., Bendixen, T., Berniūnas, R., Cheshin, A., Gentili, C., Georgescu, R., Gervais, W. M., Hagel, K., Kavanagh, C., Levy, N., Neely, A., Qiu, L., Rabelo, A., Ramsay, J. E., ... van Elk, M. (2021). The Einstein effect: Global evidence for scientific source credibility effects and the influence of religiosity. PsyArXiv. https://doi.org/10.31234/osf.io/sf8ez 
Hoogeveen, S., Sarafoglou, A., van Elk, M., \& Wagenmakers, E.-J. (in preparation). A manyanalysts approach to the relation between religiosity and well-being: Discussion and conclusions.

Hoogeveen, S., \& van Elk, M. (2018). Advancing the Cognitive Science of Religion through Replication and Open Science. Journal for the Cognitive Science of Religion, 6(1-2), 158-190. https://doi.org/10.1558/jcsr.39039

Huijts, T., \& Kraaykamp, G. (2011). Religious involvement, religious context, and selfassessed health in Europe. Journal of Health and Social Behavior, 52(1), 91-106. https://doi.org/10.1177/0022146510394950

Huntington-Klein, N., Arenas, A., Beam, E., Bertoni, M., Bloem, J. R., Burli, P., Chen, N., Grieco, P., Ekpe, G., Pugatch, T., Saavedra, M., \& Stopnitzky, Y. (2021). The influence of hidden researcher decisions in applied microeconomics. Economic Inquiry, 59(3), 944-960. https://doi.org/10.1111/ecin.12992

Kelley, J., \& de Graaf, N. D. (1997). National context, parental socialization, and religious belief: Results from 15 nations. American Sociological Review, 62(4), 639-659. https: //doi.org/10.2307/2657431

Koenig, H. G. (2009). Research on religion, spirituality, and mental health: A review. The Canadian Journal of Psychiatry, 54, 283-291. https://doi.org/10.1177/ 070674370905400502

Koenig, H. G., Al-Zaben, F., \& VanderWeele, T. J. (2020). Religion and psychiatry: Recent developments in research. BJPsych Advances, 26(5), 262-272. https://doi.org/10. 1192/bja.2019.81

Koenig, H. G., Hill, T. D., Pirutinsky, S., \& Rosmarin, D. H. (2021). Commentary on "Does spirituality or religion positively affect mental health?" The International Journal for the Psychology of Religion, 31(1), 27-44. https://doi.org/10.1080/10508619. 2020.1766868 
Koenig, H. G., \& Larson, D. B. (2001). Religion and mental health: Evidence for an association. International Review of Psychiatry, 13(2), 67-78. https://doi.org/10.1080/ 09540260124661

Kvarven, A., Strømland, E., \& Johannesson, M. (2020). Comparing meta-analyses and preregistered multiple-laboratory replication projects. Nature Human Behaviour, 4(4), 423-434. https://doi.org/10.1038/s41562-019-0787-z

Ladd, K. L., \& Messick, K. J. (2016). A brief history of the psychological study of the role(s) of religion. Psychological specialties in historical context: Enriching the classroom experience for teachers and students. (pp. 204-216).

Lewis, C. A., \& Cruise, S. M. (2006). Religion and happiness: Consensus, contradictions, comments and concerns. Mental Health, Religion $\& 3$ Culture, 9(3), 213-225. https: //doi.org/10.1080/13694670600615276

Lim, C., \& Putnam, R. D. (2010). Religion, social networks, and life satisfaction. American Sociological Review, 75(6), 914-933. https://doi.org/10.1177/0003122410386686

Luo, W., \& Chen, F. (2021). The salience of religion under an atheist state: Implications for subjective well-being in contemporary China. Social Forces, 100(2), 852-878. https://doi.org/10.1093/sf/soab049

Maier-Hein, K. H., Neher, P. F., Houde, J.-C., Côté, M.-A., Garyfallidis, E., Zhong, J., Chamberland, M., Yeh, F.-C., Lin, Y.-C., Ji, Q., Reddick, W. E., Glass, J. O., Chen, D. Q., Feng, Y., Gao, C., Wu, Y., Ma, J., He, R., Li, Q., .. Descoteaux, M. (2017). The challenge of mapping the human connectome based on diffusion tractography. Nature Communications, 8, 1349. https://doi.org/10.1038/s41467-017-01285-x

May, M., \& Smilde, D. (2016). Minority participation and well-being in majority Catholic nations: What does it mean to be a religious minority? Journal of Religion and Health, 55 (3), 874-894. https://doi.org/10.1007/s10943-015-0099-1

Okulicz-Kozaryn, A. (2010). Religiosity and life satisfaction across nations. Mental Health, Religion $\&$ Culture, 13(2), 155-169. https://doi.org/10.1080/13674670903273801 
Plante, T. G., \& Sherman, A. C. (2001). Faith and health: Psychological perspectives. Guilford Press.

Poloma, M. M., \& Pendleton, B. F. (1989). Exploring types of prayer and quality of life: A research note. Review of Religious Research, 31(1), 46-53. https://doi.org/10.2307/ 3511023

Rohrer, J. M. (2018). Thinking clearly about correlations and causation: Graphical causal models for observational data. Advances in Methods and Practices in Psychological Science, 1(1), 27-42. https://doi.org/10.1177/2515245917745629

Rosmarin, D. H., Pargament, K. I., Pirutinsky, S., \& Mahoney, A. (2010). A randomized controlled evaluation of a spiritually integrated treatment for subclinical anxiety in the Jewish community, delivered via the Internet. Journal of Anxiety Disorders, 24(7), 799-808. https://doi.org/10.1016/j.janxdis.2010.05.014

Ruiter, S., \& van Tubergen, F. (2009). Religious attendance in cross-national perspective: A multilevel analysis of 60 countries. American Journal of Sociology, 115(3), 863-895. https://doi.org/10.1086/603536

Salganik, M. J., Lundberg, I., Kindel, A. T., Ahearn, C. E., Al-Ghoneim, K., Almaatouq, A., Altschul, D. M., Brand, J. E., Carnegie, N. B., Compton, R. J., Datta, D., Davidson, T., Filippova, A., Gilroy, C., Goode, B. J., Jahani, E., Kashyap, R., Kirchner, A., McKay, S., ... McLanahan, S. (2020). Measuring the predictability of life outcomes with a scientific mass collaboration. Proceedings of the National Academy of Sciences, 117(15), 8398-8403. https://doi.org/10.1073/pnas.1915006117

Sarafoglou, A., Hoogeveen, S., \& Wagenmakers, E.-J. (2022). Comparing analysis blinding with preregistration in the many-analysts religion project. Manuscript submitted for publication. https://doi.org/10.31234/osf.io/6dn8f

Schweinsberg, M., Feldman, M., Staub, N., van den Akker, O. R., van Aert, R. C. M., van Assen, M. A. L. M., Liu, Y., Althoff, T., Heer, J., Kale, A., Mohamed, Z., Amireh, H., Venkatesh Prasad, V., Bernstein, A., Robinson, E., Snellman, K., Amy Sommer, S., Otner, S. M. G., Robinson, D., ... Luis Uhlmann, E. (2021). Same 
data, different conclusions: Radical dispersion in empirical results when independent analysts operationalize and test the same hypothesis. Organizational Behavior and Human Decision Processes, 165, 228-249. https://doi.org/10.1016/j.obhdp.2021. 02.003

Seybold, K. S., \& Hill, P. C. (2001). The role of religion and spirituality in mental and physical health. Current Directions in Psychological Science, 10(1), 21-24. https: //doi.org/10.1111/1467-8721.00106

Silberzahn, R., \& Uhlmann, E. L. (2015). Many hands make tight work. Nature, 526, 189191.

Silberzahn, R., Uhlmann, E. L., Martin, D. P., Anselmi, P., Aust, F., Awtrey, E., Bahnik, Š., Bai, F., Bannard, C., Bonnier, E., et al. (2018). Many analysts, one data set: Making transparent how variations in analytic choices affect results. Advances in Methods and Practices in Psychological Science, 1, 337-356. https://doi.org/10. $1177 / 2515245917747646$

Simkin, H. (2020). The centrality of events, religion, spirituality, and subjective well-being in Latin American Jewish immigrants in Israel. Frontiers in Psychology, 11, 576402. https://doi.org/10.3389/fpsyg.2020.576402

Smith, T. B., McCullough, M. E., \& Poll, J. (2003). Religiousness and depression: Evidence for a main effect and the moderating influence of stressful life events. Psychological Bulletin, 129(4), 614-636. https://doi.org/10.1037/0033-2909.129.4.614

Starns, J. J., Cataldo, A. M., Rotello, C. M., Annis, J., Aschenbrenner, A., Bröder, A., Cox, G., Criss, A., Curl, R. A., Dobbins, I. G., Dunn, J., Enam, T., Evans, N. J., Farrell, S., Fraundorf, S. H., Gronlund, S. D., Heathcote, A., Heck, D. W., Hicks, J. L., ... Wilson, J. (2019). Assessing theoretical conclusions with blinded inference to investigate a potential inference crisis. Advances in Methods and Practices in Psychological Science, 2(4), 335-349. https://doi.org/10.1177/2515245919869583 
Stavrova, O. (2015). Religion, self-rated health, and mortality: Whether religiosity delays death depends on the cultural context. Social Psychological and Personality Science, 6(8), 911-922. https://doi.org/10.1177/1948550615593149

Stavrova, O., Fetchenhauer, D., \& Schlösser, T. (2013). Why are religious people happy? The effect of the social norm of religiosity across countries. Social Science Research, 42(1), 90-105. https://doi.org/10.1016/j.ssresearch.2012.07.002

Steegen, S., Tuerlinckx, F., Gelman, A., \& Vanpaemel, W. (2016). Increasing transparency through a multiverse analysis. Perspectives on Psychological Science, 11(5), 702712. https://doi.org/10.1177/1745691616658637

Swigart, K. L., Anantharaman, A., Williamson, J. A., \& Grandey, A. A. (2020). Working while liberal/conservative: A review of political ideology in organizations. Journal of Management, 46(6), 1063-1091. https://doi.org/10.1177/0149206320909419

Thoresen, C. E. (1999). Spirituality and health: Is there a relationship? Journal of Health Psychology, 4(3), 291-300. https://doi.org/10.1177/135910539900400314

van Doorn, J., Ly, A., Marsman, M., \& Wagenmakers, E.-J. (2020). Bayesian rank-based hypothesis testing for the rank sum test, the signed rank test, and Spearman's $\rho$. Journal of Applied Statistics, 47(16), 2984-3006. https://doi.org/10.1080/02664763. 2019.1709053

van Elk, M. (2021). Assessing the religion-health relationship: Introduction to the metaanalysis by Garssen et al., and two commentaries. The International Journal for the Psychology of Religion, 31(1), 1-3. https://doi.org/10.1080/10508619.2021.1877029

van Elk, M., Matzke, D., Gronau, Q., Guang, M., Vandekerckhove, J., \& Wagenmakers, E.-J. (2015). Meta-analyses are no substitute for registered replications: A skeptical perspective on religious priming. Frontiers in Psychology, 6, 1365. https://doi.org/ 10.3389/fpsyg.2015.01365

van Dongen, N. N. N., van Doorn, J. B., Gronau, Q. F., van Ravenzwaaij, D., Hoekstra, R., Haucke, M. N., Lakens, D., Hennig, C., Morey, R. D., Homer, S., Gelman, A., Sprenger, J., \& Wagenmakers, E.-J. (2019). Multiple perspectives on inference 
for two simple statistical scenarios. The American Statistician, 73(sup1), 328-339. https://doi.org/10.1080/00031305.2019.1565553

Villani, D., Sorgente, A., Iannello, P., \& Antonietti, A. (2019). The role of spirituality and religiosity in subjective well-being of individuals with different religious status. Frontiers in Psychology, 10. https://doi.org/10.3389/fpsyg.2019.01525

Wan, C., Chiu, C.-y., Tam, K.-P., Lee, S.-l., Lau, I. Y.-m., \& Peng, S. (2007). Perceived cultural importance and actual self-importance of values in cultural identification. Journal of Personality and Social Psychology, 92(2), 337-354. https://doi.org/10. 1037/0022-3514.92.2.337

Weber, S. R., \& Pargament, K. I. (2014). The role of religion and spirituality in mental health. Current Opinion in Psychiatry, 27(5), 358-363. https://doi.org/10.1097/ YCO.0000000000000080

WHOQOL Group. (1998). Development of the World Health Organization WHOQOLBREF Quality of Life Assessment. Psychological Medicine, 28(3), 551-558. https: //doi.org/10.1017/s0033291798006667

Wiebe, D. (2009). Religious biases in funding religious studies research? Religio: Revue pro Religionistiku, 17(2), 125-140.

World Bank Group. (2017). World Bank Group - International Development, Poverty, \& Sustainability.

World Values Survey. (2010). Wave 6 Official Aggregate v. 20150418.

Wulff, D. M. (1998). Rethinking the rise and fall of the psychology of religion. Religion in the Making, 181-202. https://doi.org/10.1163/9789004379039_013

Zimmer, Z., Jagger, C., Chiu, C.-T., Ofstedal, M. B., Rojo, F., \& Saito, Y. (2016). Spirituality, religiosity, aging and health in global perspective: A review. SSM - Population Health, 2, 373-381. https://doi.org/10.1016/j.ssmph.2016.04.009

Zou, X., Tam, K.-P., Morris, M. W., Lee, S.-l., Lau, I. Y.-M., \& Chiu, C.-y. (2009). Culture as common sense: Perceived consensus versus personal beliefs as mechanisms of 
cultural influence. Journal of Personality and Social Psychology, 97(4), 579-597. https://doi.org/10.1037/a0016399 


\section{Appendix A}

\section{Variable Inclusion}

Here we show the variable inclusion for each team; Table A1 displays the included items for the dependent variable for research question 1 and Table A2 displays the included items for the dependent variable for research question 2. Table A3 displays the included items for the independent variable for research question 1 and Table A4 displays the included items for the independent variable for research question 2. Table A5 displays the included items as covariates for research question 1 and Table A6 displays the included items as covariates for research question 2.

\section{Table A1}

Items Included as Dependent Variables for Research Question 1 by Each Team.

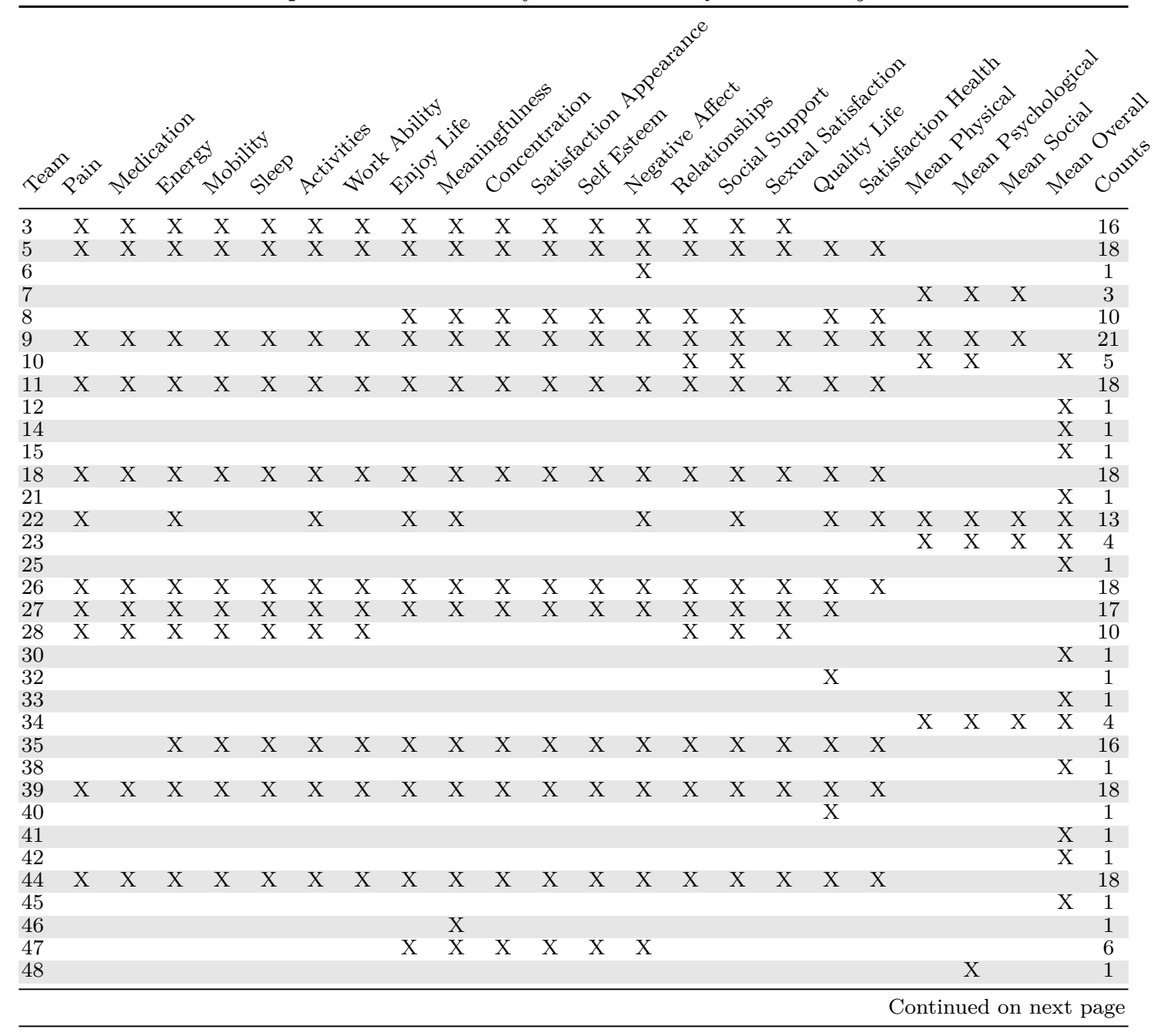




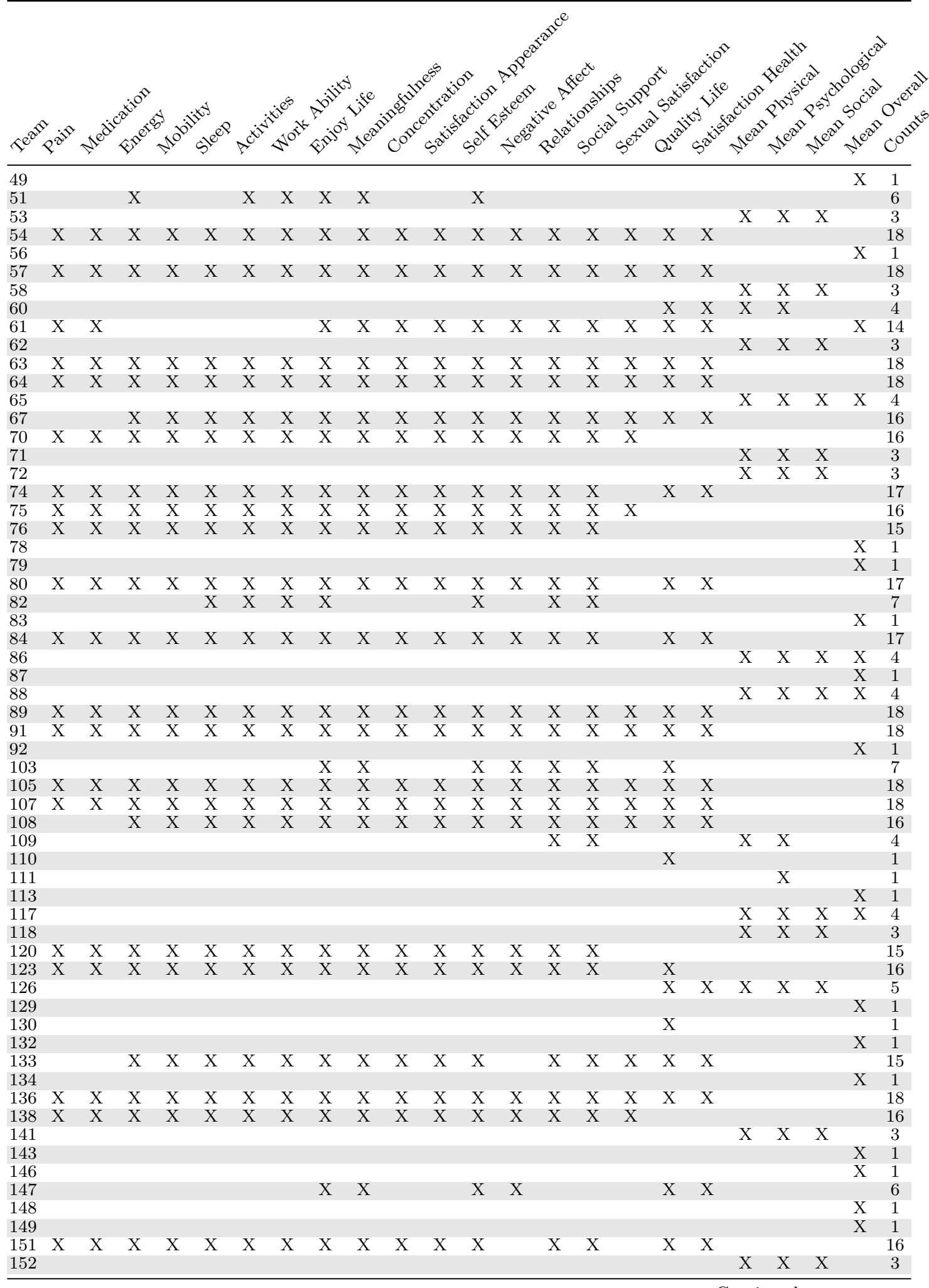




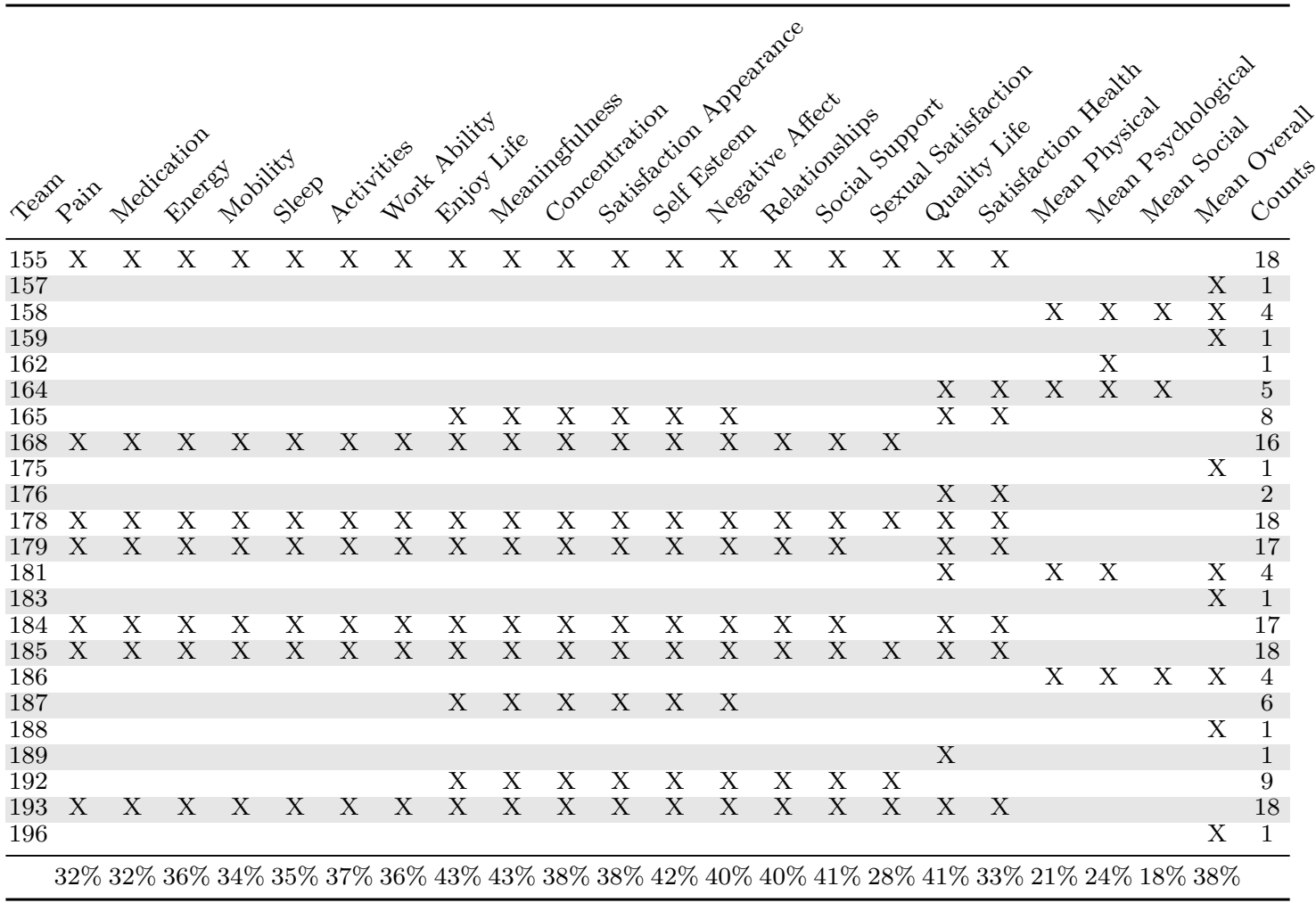

Table A2

Items Included as Dependent Variables for Research Question 2 by Each Team.

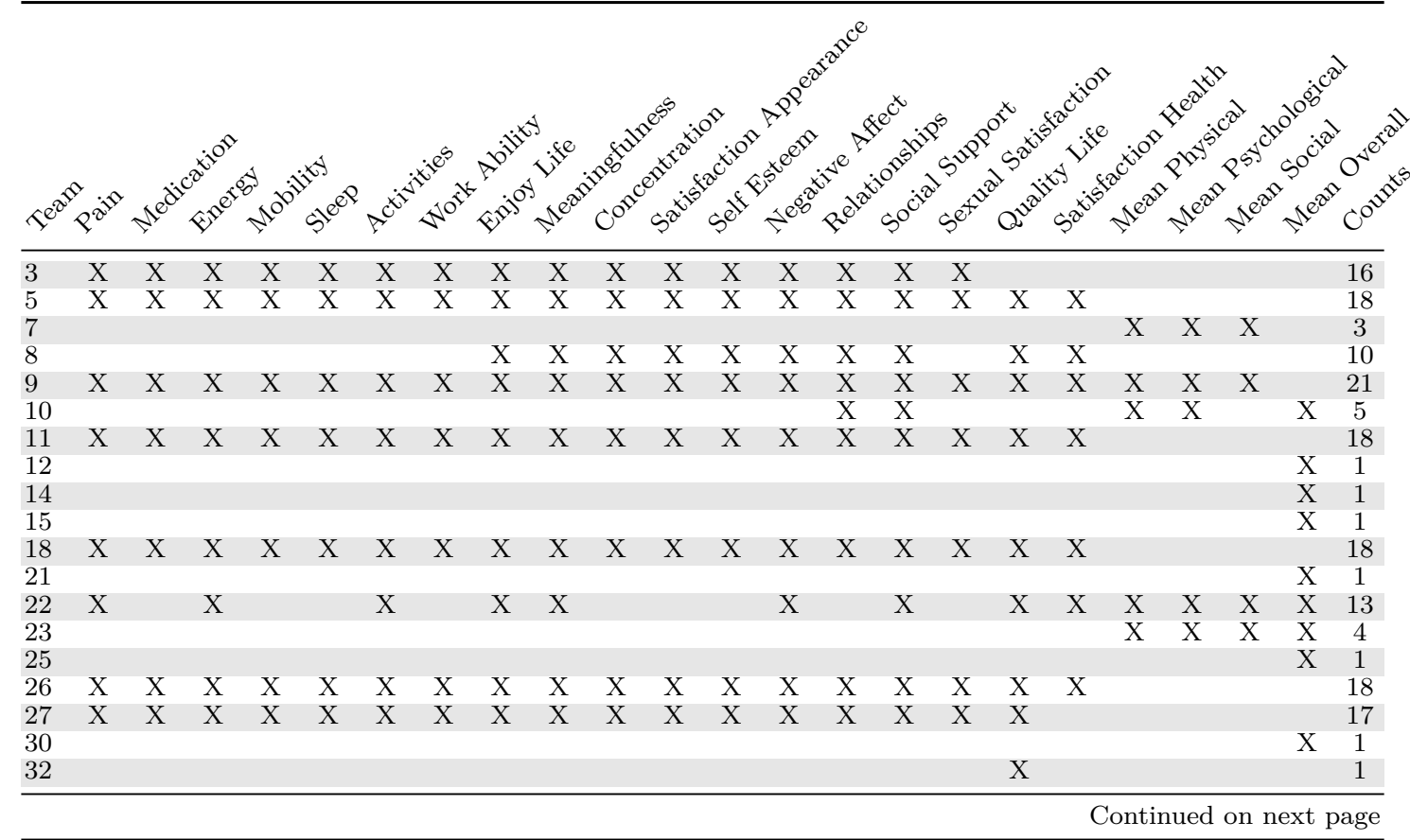

Continued on next page 


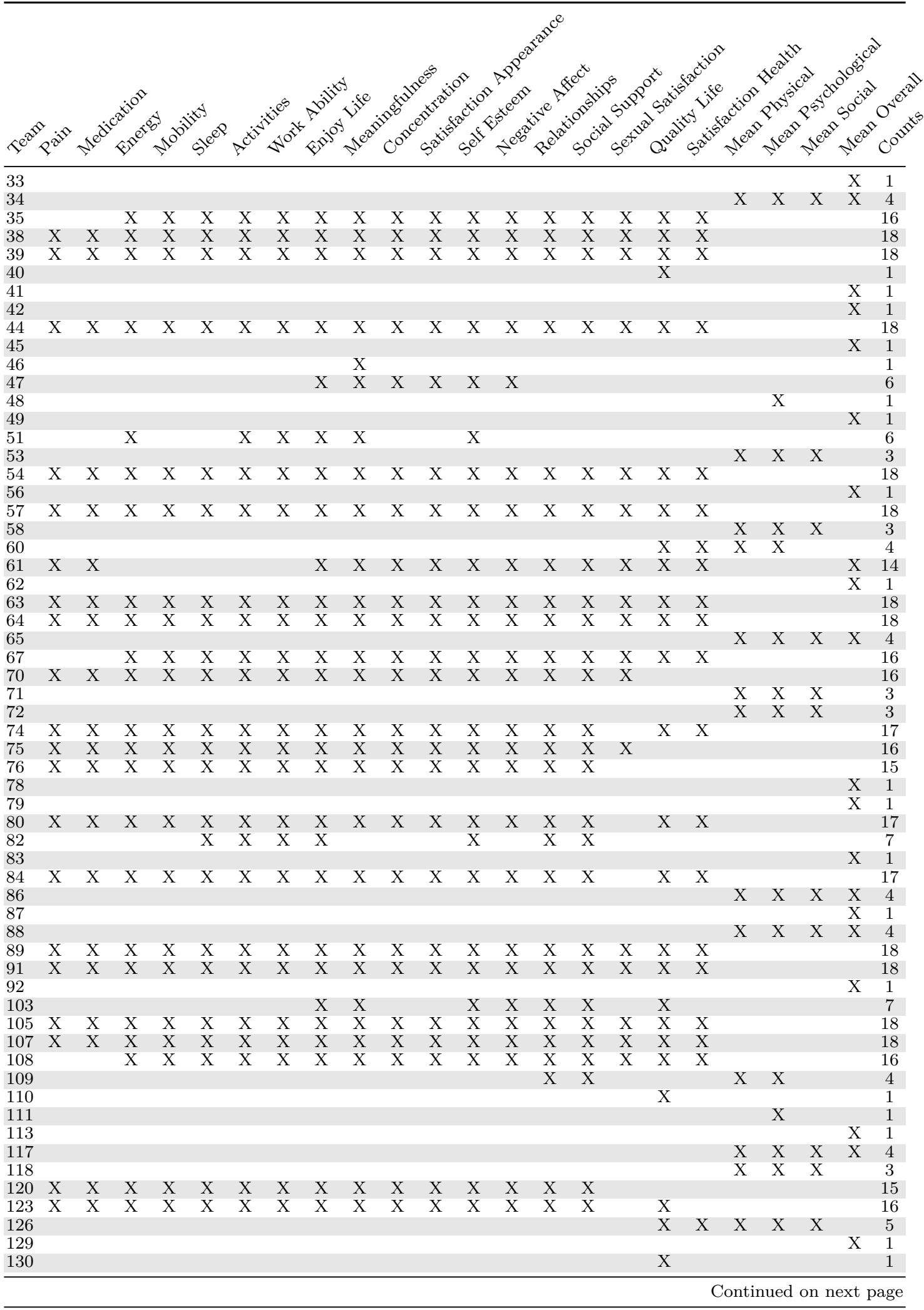




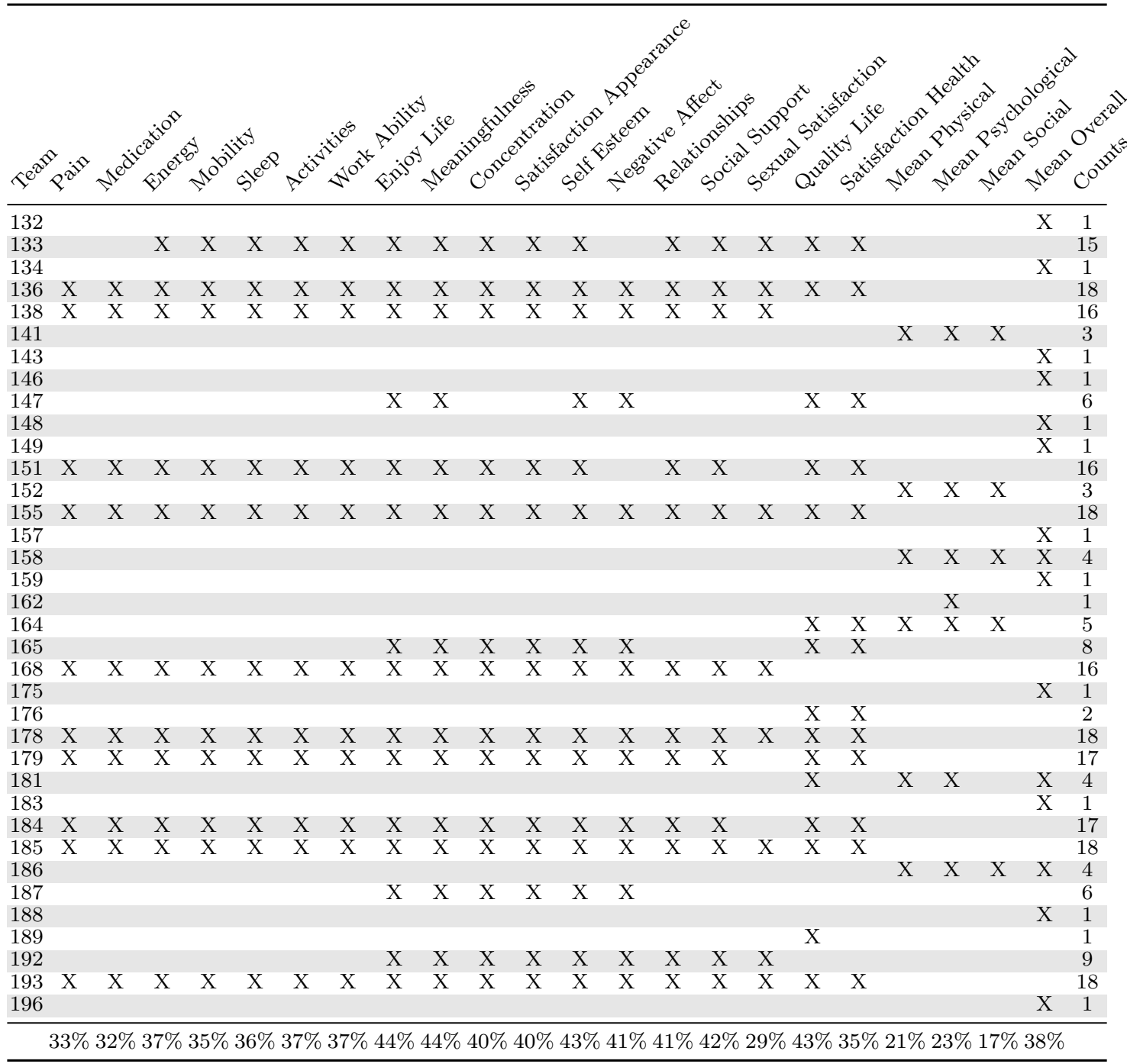

\section{Table A3}

Items Included as Independent Variables for Research Question 1 by Each Team.

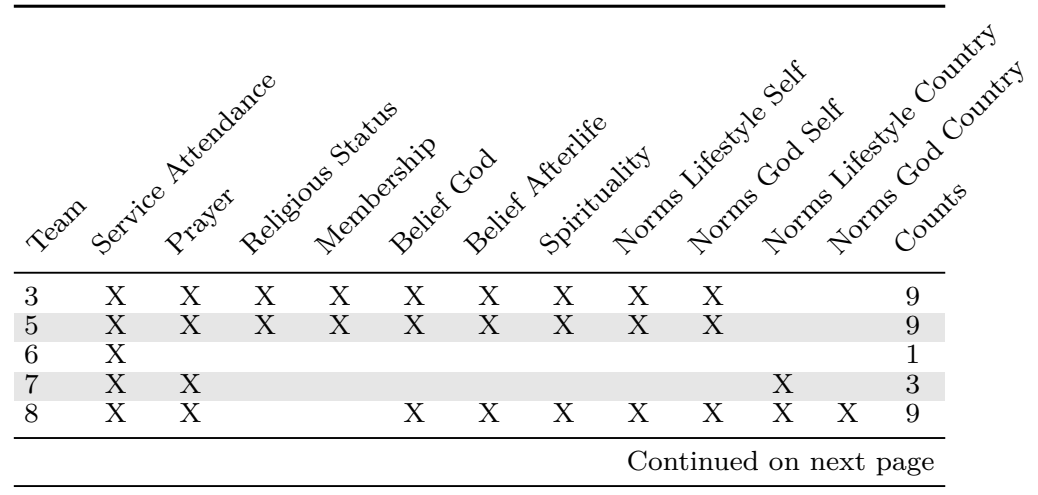


Table A3 continued

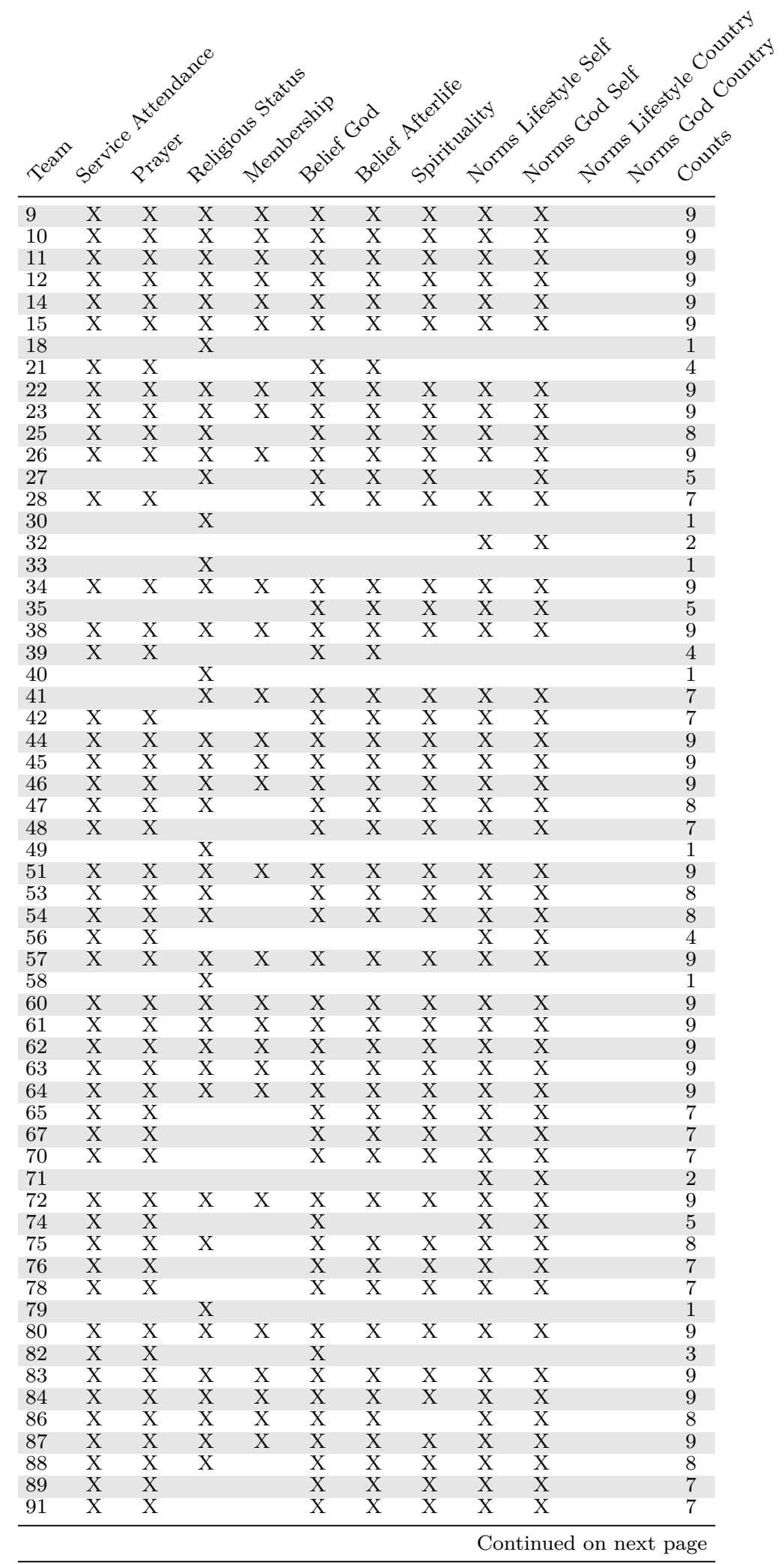




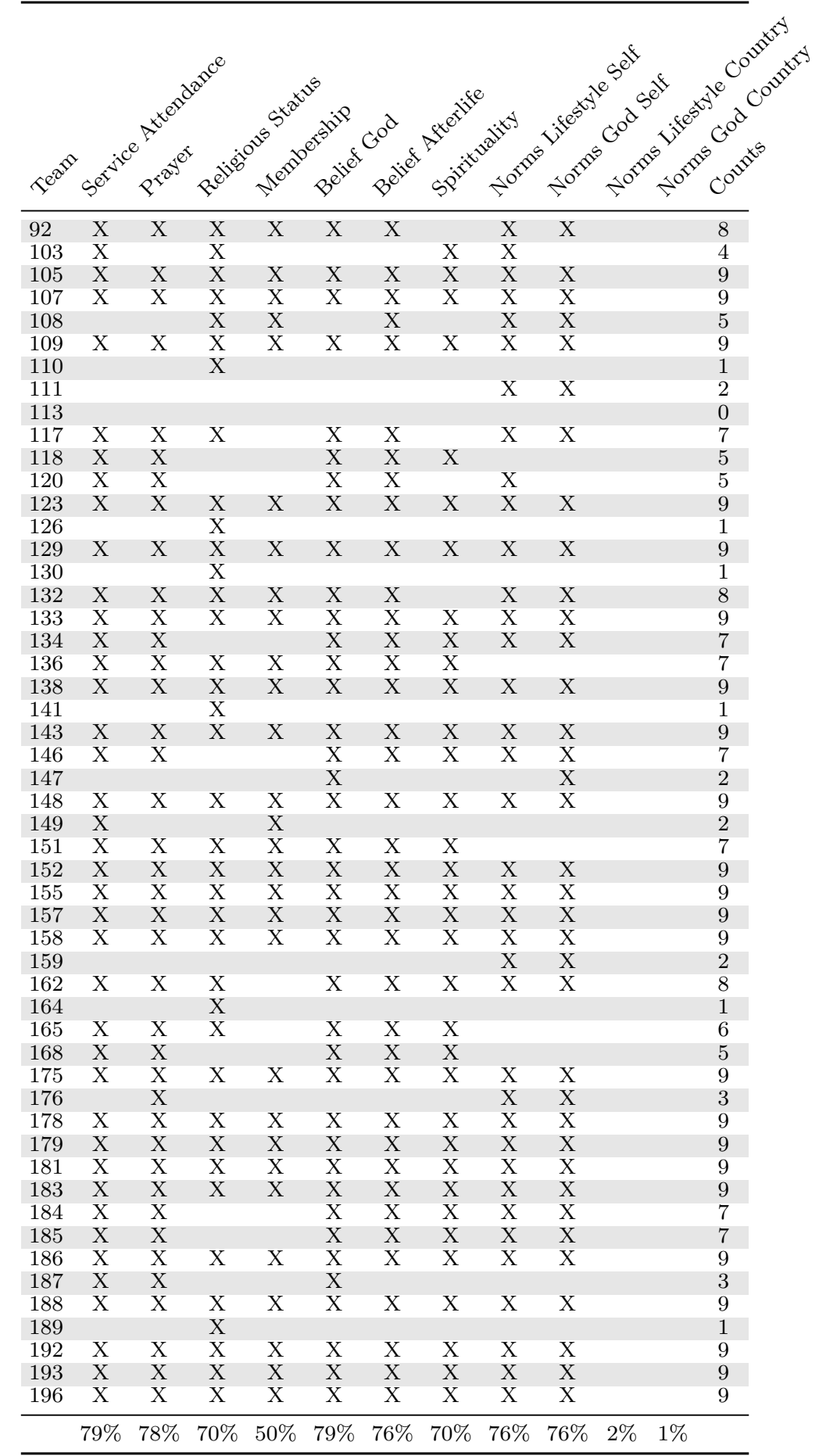


Table A4

Items Included as Independent Variables for Research Question 2 by Each Team.

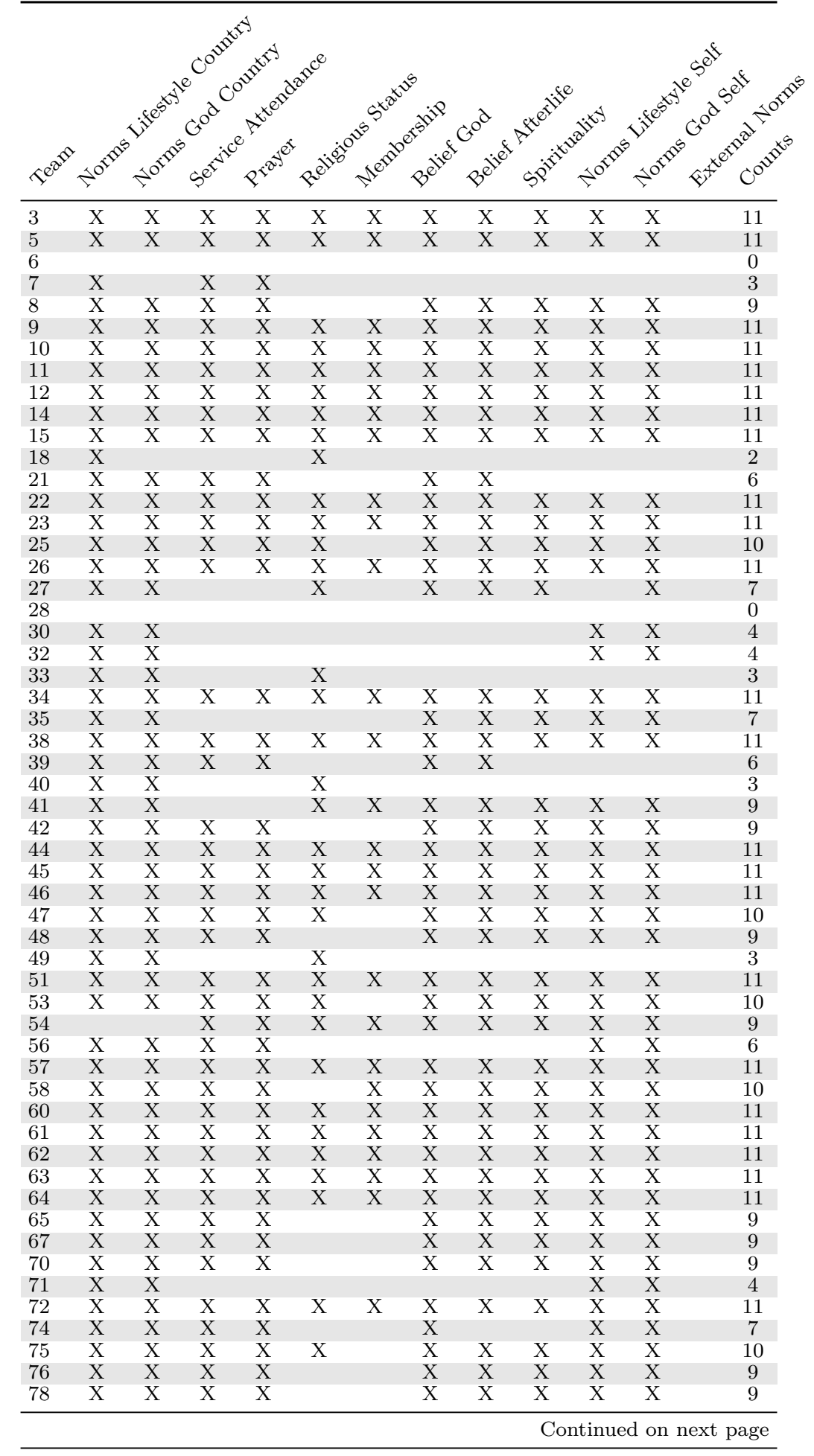


Table A4 continued

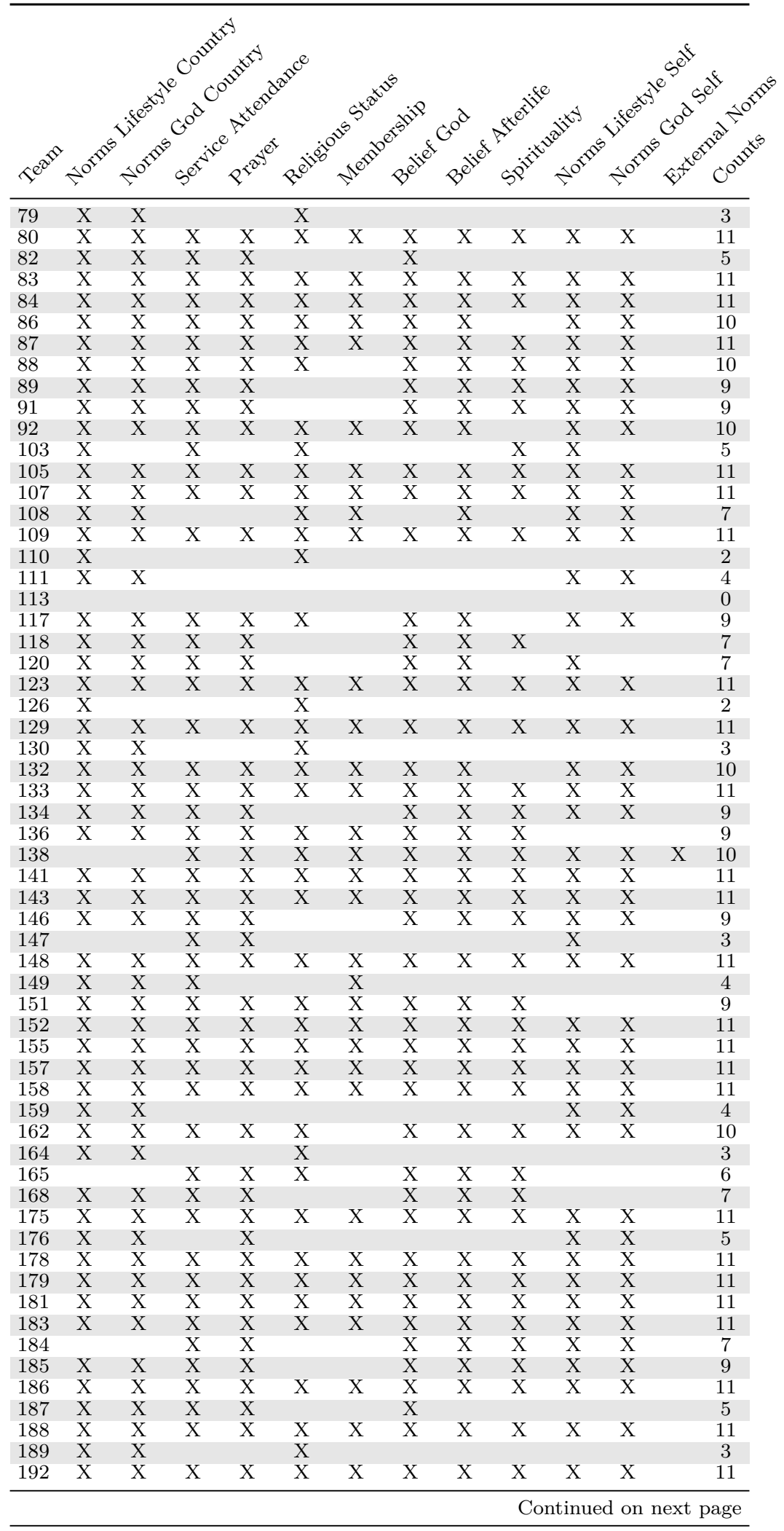


Table A4 continued

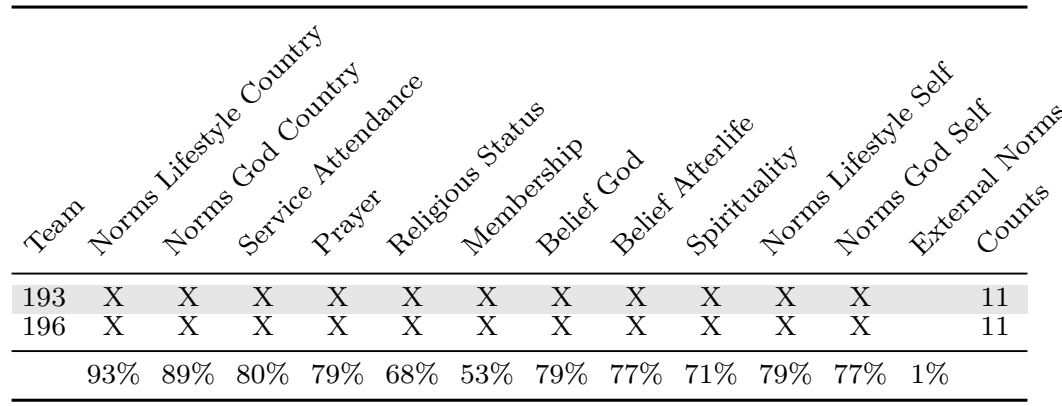

Note. Variables indicated as 'External' refer to variables that are based on data not provided by the MARP team.

\section{Table A5}

\section{Covariates Included for Research Question 1 by Each Team.}




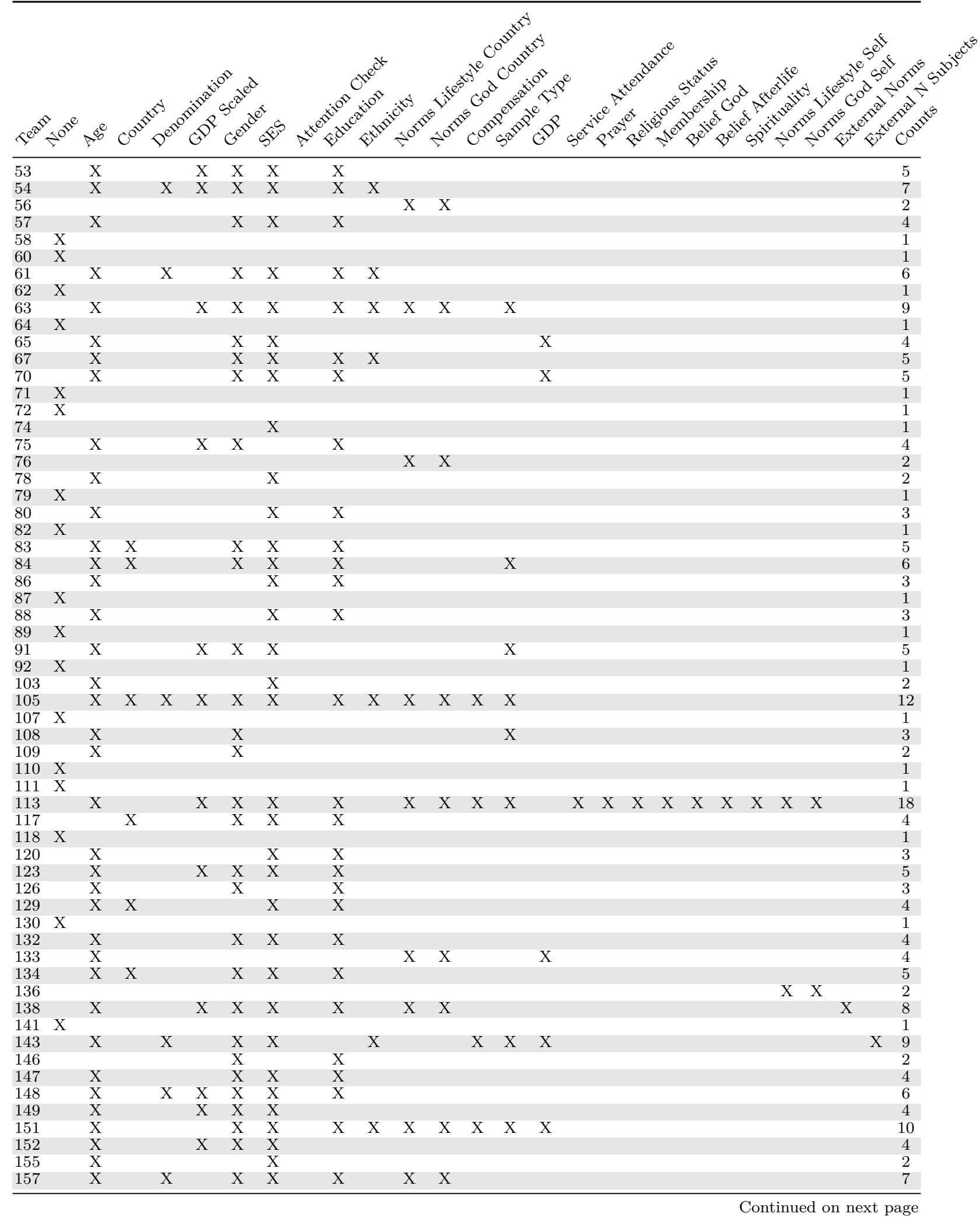




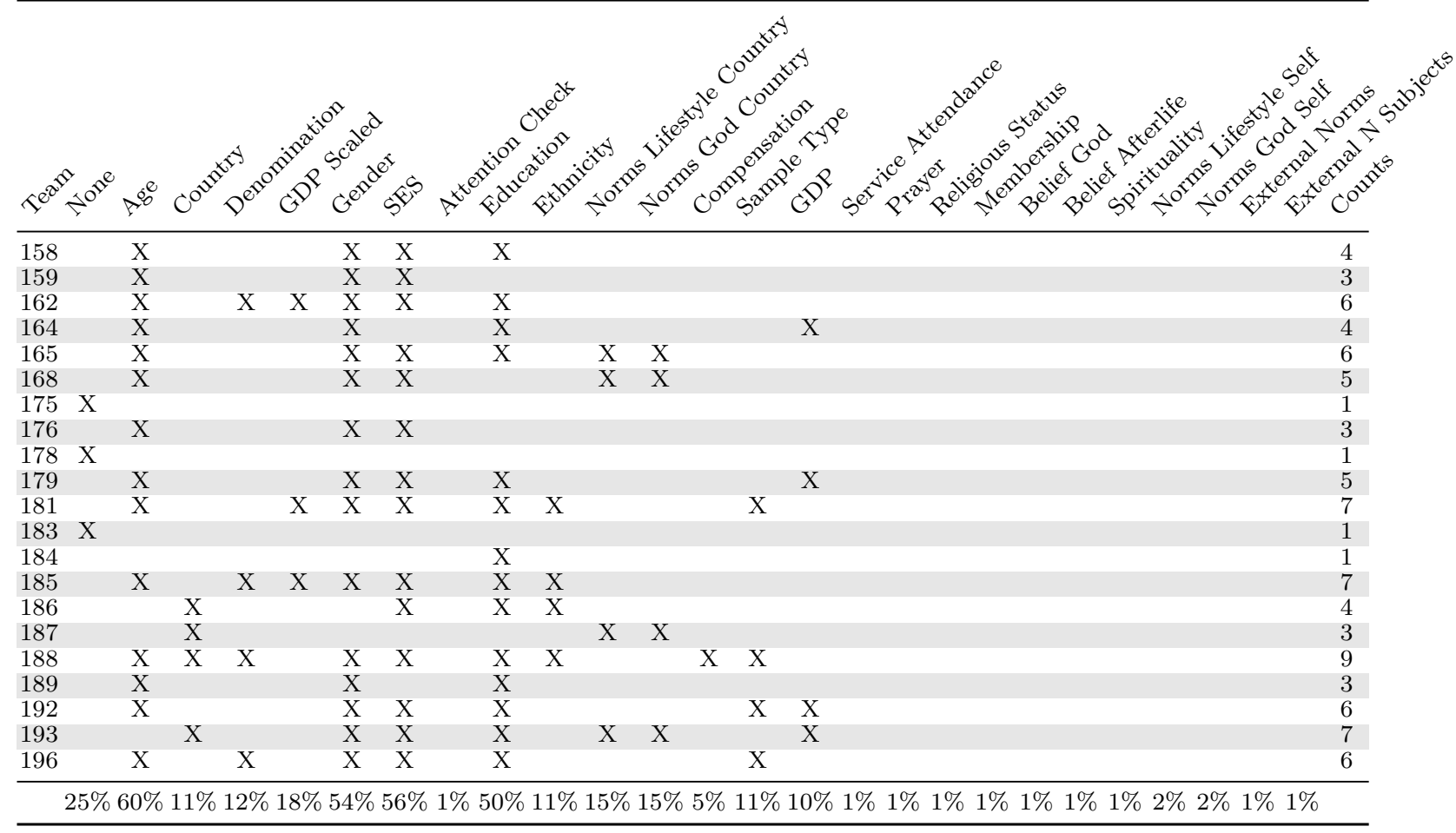

Note. Variables indicated as 'External' refer to covariates that are based on data not provided by the MARP team.

\section{Table A6}

Covariates Included for Research Question 2 by Each Team.

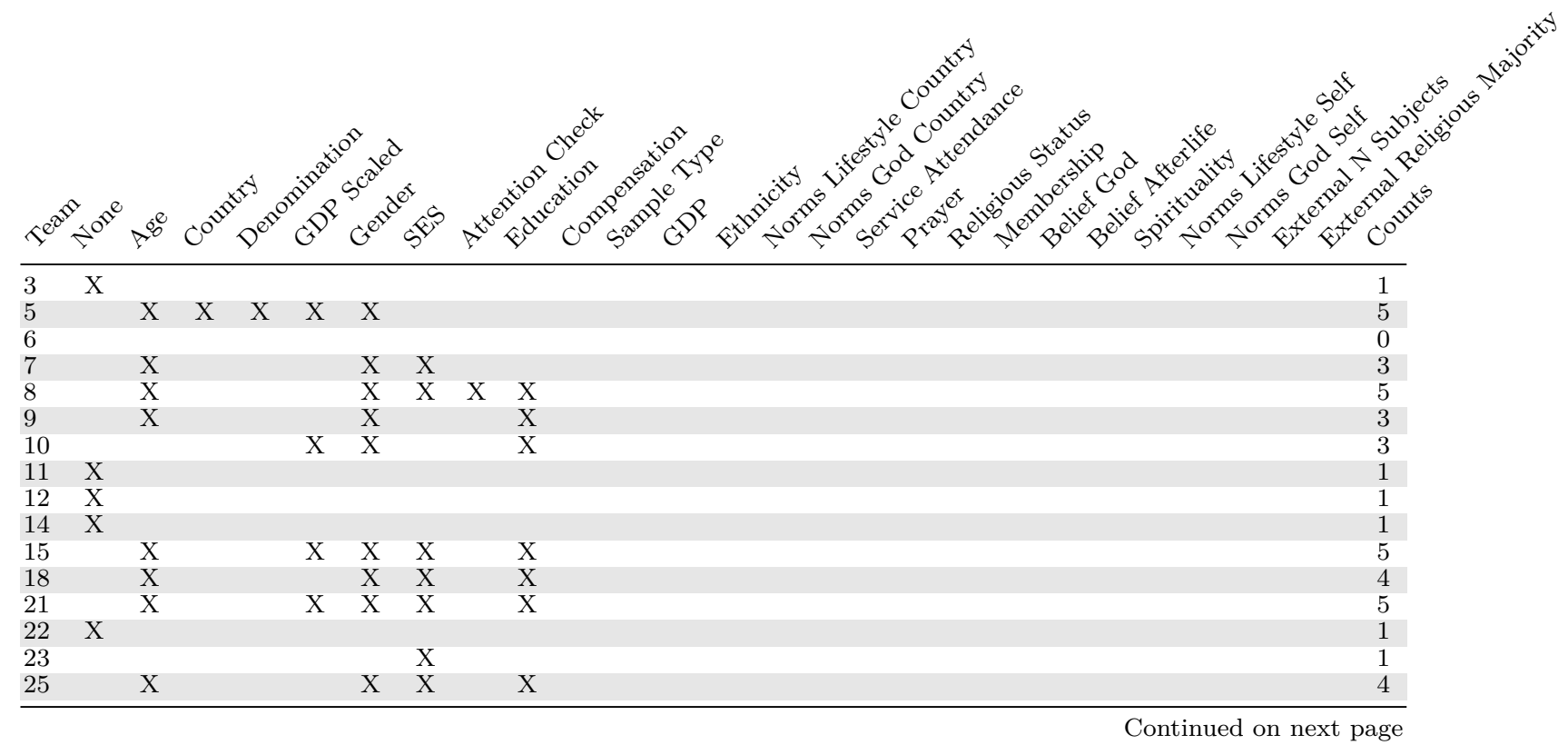




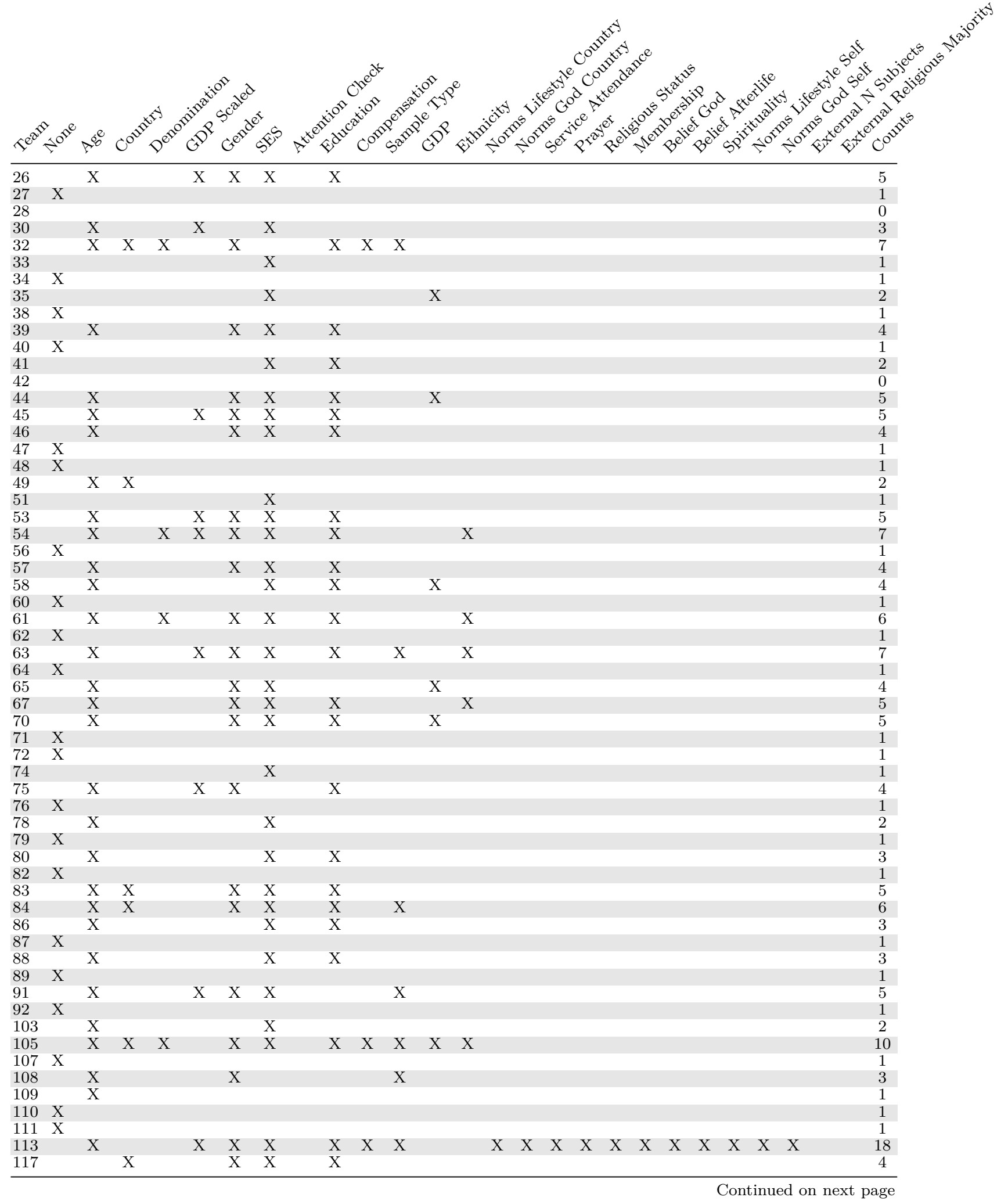




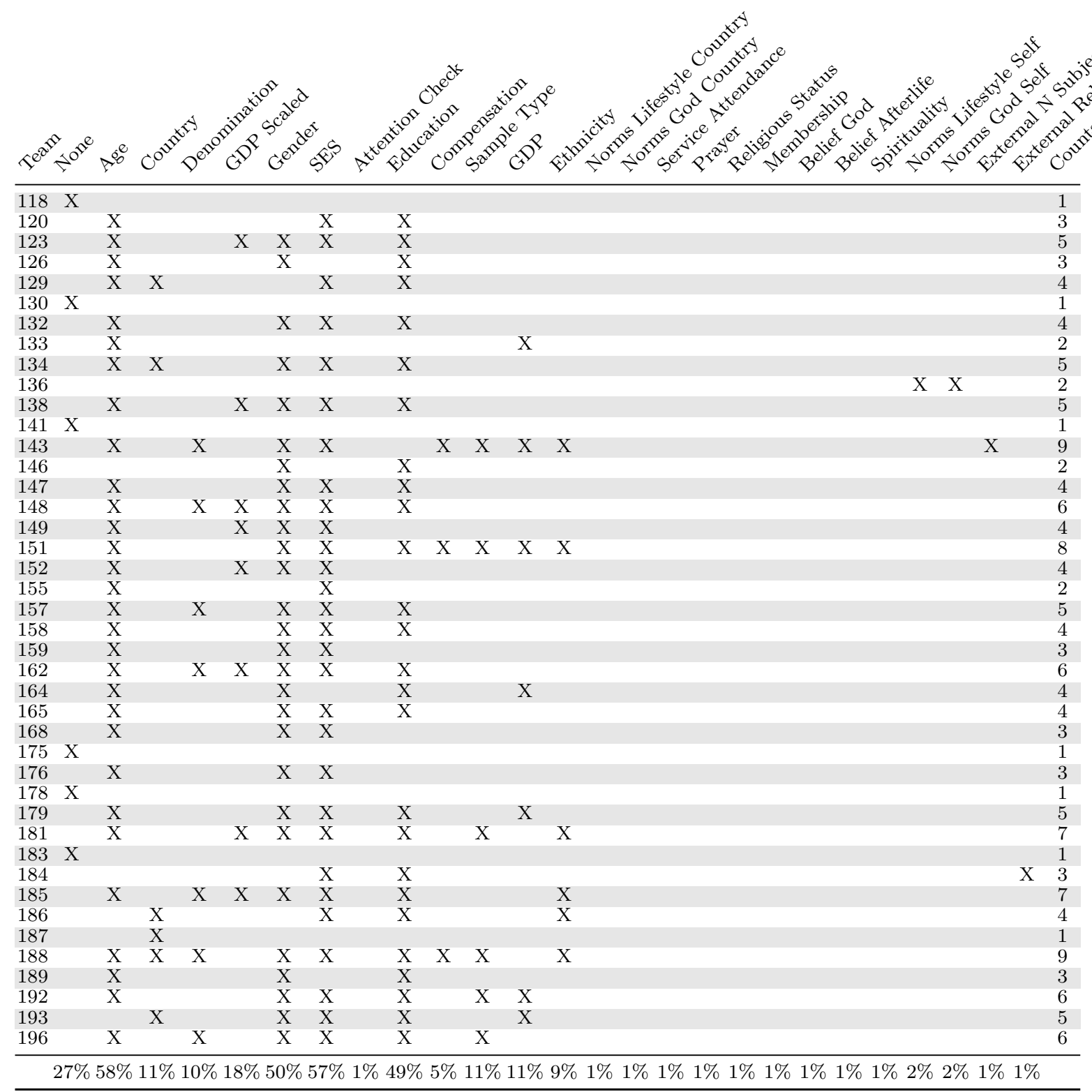

Note. Variables indicated as 'External' refer to covariates that are based on data not provided by the MARP team. 


\section{Ethical Approval Cross-cultural Data Collection}

Data collection for the cross-cultural study was approved by the local ethics committee at the Psychology Department of the University of Amsterdam (Project \#2018-SP9713). Additional approval was obtained from local IRBs at the Adolfo Ibáñez University (Chile), the Babes-Bolyai University (Romania), the James Cook University (Singapore), Royal Holloway, University of London (UK), the University of Connecticut (US), and the Max Planck Society, as well as the Senate Department for Education, Youth and Fam-

ily from the Ministry of Education in Berlin (Germany). All participants were treated in accordance with the Declaration of Helsinki. 


\section{Table B1}

Descriptive Statistics of the Cross-cultural Data

\begin{tabular}{lcccccc}
\hline Country & $\mathrm{N}$ & Age (SD) & Women & Well-being & Religiosity & Cultural norms \\
\hline Australia & 482 & $47.8(15.9)$ & $47.9 \%$ & 3.7 & 0.53 & 0.46 \\
Belgium & 325 & $34.9(13.4)$ & $54.8 \%$ & 3.8 & 0.27 & 0.22 \\
Brazil & 406 & $29.1(10.8)$ & $73.2 \%$ & 3.5 & 0.51 & 0.61 \\
Canada & 351 & $33.2(10.5)$ & $52.4 \%$ & 3.6 & 0.30 & 0.29 \\
Chile & 308 & $30.8(9.9)$ & $59.1 \%$ & 3.7 & 0.36 & 0.44 \\
China & 420 & $32.1(8.5)$ & $55.0 \%$ & 3.6 & 0.33 & 0.31 \\
Croatia & 311 & $28.0(6.8)$ & $77.8 \%$ & 3.8 & 0.44 & 0.58 \\
Denmark & 420 & $27.9(10.4)$ & $70.7 \%$ & 3.8 & 0.29 & 0.19 \\
France & 409 & $40.5(12.8)$ & $63.8 \%$ & 3.6 & 0.32 & 0.30 \\
Germany & 1,306 & $27.6(9.1)$ & $62.2 \%$ & 3.8 & 0.36 & 0.35 \\
India & 454 & $30.4(6.4)$ & $37.9 \%$ & 3.8 & 0.74 & 0.70 \\
Ireland & 434 & $42.6(15.0)$ & $51.8 \%$ & 3.6 & 0.49 & 0.46 \\
Israel & 514 & $28.1(10.5)$ & $73.0 \%$ & 3.8 & 0.36 & 0.49 \\
Italy & 347 & $27.2(8.3)$ & $50.1 \%$ & 3.5 & 0.27 & 0.47 \\
Japan & 427 & $40.5(10.0)$ & $43.6 \%$ & 3.0 & 0.29 & 0.28 \\
Lithuania & 297 & $24.1(6.9)$ & $82.5 \%$ & 3.5 & 0.37 & 0.43 \\
Morocco & 397 & $33.9(13.1)$ & $16.1 \%$ & 3.6 & 0.71 & 0.75 \\
Netherlands & 499 & $58.0(14.7)$ & $25.1 \%$ & 3.9 & 0.29 & 0.29 \\
Romania & 580 & $25.0(8.2)$ & $84.1 \%$ & 3.8 & 0.59 & 0.59 \\
Singapore & 327 & $22.2(3.5)$ & $62.4 \%$ & 3.5 & 0.46 & 0.39 \\
Spain & 341 & $41.9(13.9)$ & $31.4 \%$ & 3.7 & 0.25 & 0.28 \\
Turkey & 367 & $39.3(11.2)$ & $24.3 \%$ & 3.7 & 0.34 & 0.64 \\
UK & 400 & $36.2(12.7)$ & $65.8 \%$ & 3.5 & 0.25 & 0.20 \\
US & 413 & $35.6(14.4)$ & $50.6 \%$ & 3.7 & 0.48 & 0.46 \\
Total & 10,535 & $33.8(13.9)$ & $55.4 \%$ & 3.7 & 0.40 & 0.42 \\
\hline
\end{tabular}

Note. Well-being refers to the average of self-rated general (2 items), physical (7 items), psychological (6 items), and social (3 items) health, measured on a 5-point Likert scale. Religiosity refers to the average self-reported level of individual religiosity based on 9 items. Cultural norms refers to the average perceived descriptive norm of religiosity in one's country based on 2 items. Religiosity and cultural norms are transformed on a $0-1$ scale. 
Table B2

Religious Denomination in the Cross-cultural Data

\begin{tabular}{lccccccc}
\hline \multicolumn{7}{c}{ Country } & \multicolumn{7}{c}{ Religious group } \\
\cline { 2 - 7 } Christian & Muslim & Hindu & Buddhist & Jewish & Other & None \\
\hline Australia & $43.2 \%$ & $5.2 \%$ & $0.6 \%$ & $0.6 \%$ & $0.4 \%$ & $3.7 \%$ & $46.3 \%$ \\
Belgium & $28.0 \%$ & $2.8 \%$ & $0.0 \%$ & $0.6 \%$ & $0.3 \%$ & $1.2 \%$ & $67.1 \%$ \\
Brazil & $30.0 \%$ & $0.0 \%$ & $0.0 \%$ & $1.0 \%$ & $0.2 \%$ & $14.5 \%$ & $54.2 \%$ \\
Canada & $26.5 \%$ & $1.1 \%$ & $0.9 \%$ & $1.1 \%$ & $2.0 \%$ & $1.4 \%$ & $67.0 \%$ \\
Chile & $25.3 \%$ & $0.0 \%$ & $0.3 \%$ & $1.6 \%$ & $3.2 \%$ & $2.9 \%$ & $66.6 \%$ \\
China & $4.5 \%$ & $0.2 \%$ & $0.0 \%$ & $11.2 \%$ & $0.0 \%$ & $1.0 \%$ & $83.1 \%$ \\
Croatia & $54.3 \%$ & $0.3 \%$ & $0.0 \%$ & $0.6 \%$ & $0.6 \%$ & $0.6 \%$ & $43.4 \%$ \\
Denmark & $36.2 \%$ & $2.1 \%$ & $0.0 \%$ & $0.0 \%$ & $0.0 \%$ & $0.0 \%$ & $61.7 \%$ \\
France & $38.4 \%$ & $6.1 \%$ & $0.0 \%$ & $0.2 \%$ & $0.0 \%$ & $1.2 \%$ & $54.0 \%$ \\
Germany & $54.1 \%$ & $3.4 \%$ & $0.1 \%$ & $0.1 \%$ & $0.3 \%$ & $1.2 \%$ & $40.8 \%$ \\
India & $14.1 \%$ & $3.3 \%$ & $60.1 \%$ & $0.4 \%$ & $0.2 \%$ & $0.9 \%$ & $20.9 \%$ \\
Ireland & $51.6 \%$ & $1.6 \%$ & $0.2 \%$ & $0.0 \%$ & $0.2 \%$ & $3.7 \%$ & $42.6 \%$ \\
Israel & $2.5 \%$ & $3.1 \%$ & $0.0 \%$ & $0.0 \%$ & $11.9 \%$ & $1.9 \%$ & $80.5 \%$ \\
Italy & $17.9 \%$ & $0.3 \%$ & $0.0 \%$ & $0.9 \%$ & $0.0 \%$ & $0.0 \%$ & $81.0 \%$ \\
Japan & $0.9 \%$ & $0.2 \%$ & $0.0 \%$ & $15.2 \%$ & $0.0 \%$ & $1.2 \%$ & $82.4 \%$ \\
Lithuania & $39.1 \%$ & $0.0 \%$ & $0.3 \%$ & $0.0 \%$ & $0.0 \%$ & $0.7 \%$ & $59.9 \%$ \\
Morocco & $0.5 \%$ & $77.1 \%$ & $0.0 \%$ & $0.0 \%$ & $0.3 \%$ & $3.8 \%$ & $18.4 \%$ \\
Netherlands & $24.6 \%$ & $0.0 \%$ & $0.0 \%$ & $0.0 \%$ & $0.6 \%$ & $6.2 \%$ & $68.5 \%$ \\
Romania & $74.0 \%$ & $0.2 \%$ & $0.0 \%$ & $0.2 \%$ & $0.2 \%$ & $5.2 \%$ & $20.3 \%$ \\
Singapore & $17.1 \%$ & $4.9 \%$ & $4.3 \%$ & $19.6 \%$ & $0.0 \%$ & $8.6 \%$ & $45.6 \%$ \\
Spain & $40.5 \%$ & $0.0 \%$ & $0.0 \%$ & $0.0 \%$ & $0.0 \%$ & $1.2 \%$ & $58.4 \%$ \\
Turkey & $0.0 \%$ & $41.1 \%$ & $0.0 \%$ & $0.0 \%$ & $0.3 \%$ & $4.6 \%$ & $54.0 \%$ \\
UK & $22.2 \%$ & $0.5 \%$ & $0.8 \%$ & $0.5 \%$ & $0.8 \%$ & $1.0 \%$ & $74.2 \%$ \\
US & $41.6 \%$ & $1.2 \%$ & $0.5 \%$ & $0.5 \%$ & $3.1 \%$ & $5.3 \%$ & $47.7 \%$ \\
Total & $31.2 \%$ & $6.1 \%$ & $2.9 \%$ & $2.0 \%$ & $1.1 \%$ & $2.9 \%$ & $53.9 \%$ \\
\hline
\end{tabular}

Note. Percentage of people indicating to be member of the respective religious groups. Note that the response options were adjusted and further specified per country. 